\title{
FINITE QUOTIENT OF JOIN IN ALEXANDROV GEOMETRY
}

\author{
XiaOchun Rong ${ }^{1} \&$ YUSheng Wang ${ }^{2}$
}

\begin{abstract}
Given two $n_{i}$-dimensional Alexandrov spaces $X_{i}$ of curvature $\geq 1$, the join of $X_{1}$ and $X_{2}$ is an $\left(n_{1}+n_{2}+1\right)$-dimensional Alexandrov space $X$ of curvature $\geq 1$, which contains $X_{i}$ as convex subsets such that their points are $\frac{\pi}{2}$ apart. If a group acts isometrically on a join that preserves $X_{i}$, then the orbit space is called quotient of join. We show that an $n$-dimensional Alexandrov space $X$ with curvature $\geq 1$ is isometric to a finite quotient of join, if $X$ contains two compact convex subsets $X_{i}$ without boundary such that $X_{1}$ and $X_{2}$ are at least $\frac{\pi}{2}$ apart and $\operatorname{dim}\left(X_{1}\right)+$ $\operatorname{dim}\left(X_{2}\right)=n-1$.
\end{abstract}

\section{INTRODUCTION}

Consider a compact Riemannian manifold $M$ of positive sectional curvature, normalized to $\sec _{M} \geq 1$. The diameter, $\operatorname{diam}(M) \leq \pi$ (Bonnet theorem), and "=" holds if and only if $M$ is isometric to the standard sphere ([Ch]). If $\operatorname{diam}(M)>\frac{\pi}{2}$, then $M$ is homeomorphic to the standard sphere ([GS]). If $\operatorname{diam}(M)=\frac{\pi}{2}$, then the Diameter rigidity theorem ([GG]) says that $M$ is either homeomorphic to a sphere or locally isometric to a rank one symmetric space with the standard metric (cf. $[\mathrm{Wi}])$.

In this paper, we will explore a rigidity of a finite quotient of join in Alexandrov geometry (see Theorem A); which is a necessary step toward a classification for Alexandrov spaces of curvature $\geq 1$ and diameter $\frac{\pi}{2}$. Furthermore, understanding such a finite quotient of join is crucial to solve the Soul Conjecture in Alexandrov geometry by Perel'man ([Pe], [Li], [RW]).

Alexandrov geometry was introduced by Burago-Gromov-Perelman in [BGP], and extensive study has been done since then. An Alexandrov space with curvature $\geq \kappa$ is a locally compact length metric space such that every geodesic triangle looks fatter than a corresponding triangle in the simply connected 2-space form of constant curvature $\kappa$, i.e., Toponogov's comparison theorem holds (see Theorem 1.1 below).

Let $\operatorname{Alex}^{n}(\kappa)$ denote the set of all complete $n$-dimensional Alexandrov spaces with cur $\geq \kappa$. Many results in Riemannian geometry based on Toponogov's comparison theorem has been generalized to Alexandrov spaces ([BGP], cf. $[\mathrm{AKP}]$ ). Concerning a 'classification' of $X \in \operatorname{Alex}^{n}(1)$ with $\operatorname{diam}(X) \geq \frac{\pi}{2}$, the following

\footnotetext{
${ }^{1}$ Supported partially by NSF Grant DMS 1106517 and a research found from Capital normal university.

${ }^{2}$ Supported partially by NFSC 11471039 .
} 
are known: $X \in \operatorname{Alex}^{n}(1)$ satisfies $\operatorname{diam}(X) \leq \pi$, "=" holds if only only if $X$ is isometric to spherical suspension, $S(\Sigma)$ with $\Sigma \in \operatorname{Alex}^{n-1}(1)$, and if $\operatorname{diam}(X)>\frac{\pi}{2}$, then $X$ is homeomorphic to some $S(\Sigma)$ ([BGP]). Note that here $\Sigma \in \operatorname{Alex}^{n-1}(1)$ $(n \geq 4)$ can have infinitely many possible topological types, and thus unlike a complete classification in Riemannian case, the best 'classification' one can expect is to find a rigid underlying geometric structure involving Alexandrov spaces of lower dimension.

Guided by the Riemannian situation, a challenging problem is to explore a rigid structure on $X \in \operatorname{Alex}^{n}(1)$ with $\operatorname{diam}(X)=\frac{\pi}{2}$. Similar to the Riemannian case ([GG]), $X$ always has two compact convex subsets which are $\frac{\pi}{2}$-apart: let $p \in X$, then $X_{1}=\left\{x \in X|| x p \mid=\frac{\pi}{2}\right\}$ and $X_{2}=\left\{x \in X|| x X_{1} \mid=\frac{\pi}{2}\right\}$ are compact convex subsets $^{3}$, where ' $|$.$| ' denotes the distance. Let m=\operatorname{dim}\left(X_{1}\right)+\operatorname{dim}\left(X_{2}\right)$. Roughly, our main result asserts that $m \leq n-1$ and " $=$ " implies a rigid underlying geometric structure, and thus the remaining 'classification' reduces to $m \leq n-2$ (see Remark $0.5)$.

Let's now turn to a known construction for Alexandrov $n$-spaces of curvature $\geq 1([\mathrm{BGP}])$. Let $Y_{i} \in \operatorname{Alex}^{n_{i}}(1)(i=1,2)$, and $Y_{i}=\left\{p_{i}\right\}$ or $\left\{p_{i}, q_{i}\right\}$ with $\left|p_{i} q_{i}\right|=\pi$ if $n_{i}=0$. Then the following space

$$
Y_{1} * Y_{2}=Y_{1} \times Y_{2} \times\left[0, \frac{\pi}{2}\right] /\left(y_{1}, y_{2}, 0\right) \sim\left(y_{1}, y_{2}^{\prime}, 0\right),\left(y_{1}, y_{2}, \frac{\pi}{2}\right) \sim\left(y_{1}^{\prime}, y_{2}, \frac{\pi}{2}\right)
$$

equipped with the metric

$$
\cos \left|\left(y_{1}, y_{2}, t\right)\left(y_{1}^{\prime}, y_{2}^{\prime}, s\right)\right|=\cos s \cos t \cos \left|y_{1} y_{1}^{\prime}\right|+\sin s \sin t \cos \left|y_{2} y_{2}^{\prime}\right|
$$

is called the join of $Y_{1}$ and $Y_{2}$. Especially, if $Y_{1}=\{p, q\}$ with $|p q|=\pi$, then $Y_{1} * Y_{2}$ is also called a spherical suspension over $Y_{2}, S\left(Y_{2}\right)$. If $Y_{1}=\{p\}$, then the join is a half spherical suspension over $Y_{2}, S_{+}\left(Y_{2}\right)$.

It is easy to see that $Y_{1} * Y_{2} \in \operatorname{Alex}^{n}(1)$ with the following properties:

(0.1.1) $\left|p_{1} p_{2}\right|=\frac{\pi}{2}$ for all $p_{i} \in Y_{i}$.

(0.1.2) $n_{1}+n_{2}=n-1$.

(0.1.3) For $p_{i} \in Y_{i}$, there is a unique minimal geodesic jointing $p_{1}$ with $p_{2}$.

Observe that $Y_{1} * Y_{2}$ is a Riemannian manifold if and only if $Y_{i}$ and $Y_{1} * Y_{2}$ are unit spheres.

Assume that a group $\Gamma$ acts effectively on $Y_{1}$ and $Y_{2}$ by isometries. By (0.1.3), the $\Gamma$-action uniquely extends to an isometric $\Gamma$-action on $Y_{1} * Y_{2}$. The quotient space, $\left(Y_{1} * Y_{2}\right) / \Gamma \in \operatorname{Alex}^{n}(1)$ satisfies (0.1.1) and (0.1.2), but not (0.1.3). We will call $\left(Y_{1} * Y_{2}\right) / \Gamma$ a quotient of join. From the construction of a quotient of join, we observe the following properties:

(0.1.4) $\operatorname{diam}\left(\left(Y_{1} * Y_{2}\right) / \Gamma\right)=\max \left\{\frac{\pi}{2}, \operatorname{diam}\left(Y_{i} / \Gamma\right)\right\}$, and $\operatorname{dim}\left(\left(Y_{1} * Y_{2}\right) / \Gamma\right)=\frac{\pi}{2}$ if and only if $\operatorname{diam}\left(Y_{i} / \Gamma\right) \leq \frac{\pi}{2}$.

(0.1.5) If both $Y_{1}$ and $Y_{2}$ have an empty boundary ${ }^{4}$, then $\left(Y_{1} * Y_{2}\right) / \Gamma$ has an empty boundary. Note that if $\Gamma$ acts trivially on $Y_{1}$, then the joint structure on $Y_{1} * Y_{2}$ descends to a join structure on $Y_{1} *\left(Y_{2} / \Gamma\right)$, which may have an non-empty boundary.

The main result in this paper is the following rigidity of a finite quotient of join:

\footnotetext{
${ }^{3}$ We say that a subset $A \subseteq X$ is convex (resp. totally convex) if for any $x, y \in A$, there is a minimal geodesic (resp. all minimal geodesics) jointing $x$ with $y$ that is in $A$.

${ }^{4}$ If $\operatorname{dim}\left(Y_{i}\right)=0$, then that $Y_{i}$ has an empty boundary means that $Y_{i}=\left\{p_{i}, q_{i}\right\}$ with $\left|p_{i} q_{i}\right|=\pi$.
} 
Theorem A. Let $X \in \operatorname{Alex}^{n}(1)$, and let $X_{1}, X_{2}$ be two compact convex subsets in $X$ such that $\left|X_{1} X_{2}\right| \triangleq \min \left\{\left|x_{1} x_{2}\right| \mid x_{i} \in X_{i}\right\} \geq \frac{\pi}{2}$. Then

(A1) $\operatorname{dim}\left(X_{1}\right)+\operatorname{dim}\left(X_{2}\right) \leq n-1$, provided either $X_{1}$ or $X_{2}$ has an empty boundary. (A2) If both $X_{1}$ and $X_{2}$ have an empty boundary and $\operatorname{dim}\left(X_{1}\right)+\operatorname{dim}\left(X_{2}\right)=n-1$, then $X$ is isometric to a finite quotient of join (which is a join when $\Gamma=e$ ). Precisely, there is $p_{i} \in X_{i}(i=1,2)$ and a finite group $\Gamma$ acting effectively and isometrically on $\left(\Sigma_{p_{i}} X_{i}\right)^{\perp}$ such that

$$
X \stackrel{\text { isom }}{\cong}\left[\left(\Sigma_{p_{1}} X_{1}\right)^{\perp} *\left(\Sigma_{p_{2}} X_{2}\right)^{\perp}\right] / \Gamma, \quad X_{1} \stackrel{\text { isom }}{\cong}\left(\Sigma_{p_{2}} X_{2}\right)^{\perp} / \Gamma, \quad X_{2} \stackrel{\text { isom }}{\cong}\left(\Sigma_{p_{1}} X_{1}\right)^{\perp} / \Gamma,
$$

where $\Sigma_{q} X$ denotes the space of directions of $X$ at $q$, and $\left(\Sigma_{p_{i}} X_{i}\right)^{\perp}=\{u \in$ $\left.\Sigma_{p_{i}} X|| u v \mid=\frac{\pi}{2}, v \in \Sigma_{p_{i}} X_{i}\right\}$.

Note that in Theorem A there is no requirement of $\operatorname{diam}(X)=\frac{\pi}{2}$ (see (0.1.4)), and $X$ satisfying (A2) has an empty boundary (see (0.1.5)). One may review (A1) as an analog in Alexandrov geometry of Proposition 1.4 in [GG], where $X$ is a Riemannian manifold with $\sec _{X} \geq 1$ and $\operatorname{diam}(X)=\frac{\pi}{2}$.

In Riemannian case, i.e., $X$ is a Riemannian manifold, Theorem A has the following corollary.

Corollary 0.2. Let $M$ be a compact n-manifold with $\sec _{M} \geq 1$, and let $M_{i} \subset M$ be two compact totally geodesic submanifolds with $\left|M_{1} M_{2}\right| \geq \frac{\pi}{2}$. Then $\operatorname{dim}\left(M_{1}\right)+$ $\operatorname{dim}\left(M_{2}\right) \leq n-1$, and "=" implies that $M$ is isometric to a spherical space form with a quotient join structure.

Here, if $\operatorname{dim}\left(M_{i}\right)=0$, then $M_{i}$ is a single point. In Corollary $0.2, " \operatorname{dim}\left(M_{1}\right)+$ $\operatorname{dim}\left(M_{2}\right) \leq n-1$ " is also seen by Frankel's theorem ([Fr]). Indeed, Corollary 0.2 recovers the spherical space form case in the Diameter rigidity theorem in [GG]. Moreover, restricted to Riemmanian case, our approach is different from [GG]; ours relies on finding the underlying join structure (Remark 5.1).

Note that not every spherical space form satisfies Corollary 0.2 (e.g., any spherical 3-space form whose fundamental group is not cyclic). However, based on [Ro] we show that up to a finite normal covering space of a uniform bounded order, all spherical space forms satisfy Corollary 0.2 .

Corollary 0.3. There is a constant $w(n)>0$ such that any spherical $n$-space form has a normal covering space of order $\leq w(n)$ which satisfies Corollary 0.2.

Let's make a few remarks on the above results.

Remark 0.4. In the proof (A2), we construct a finite group $\Gamma$ acting isometrically on $\left(\Sigma_{p_{i}} X_{i}\right)^{\perp}$ at some $p_{i} \in X_{i}$, which extends to a unique isometric action on the join of $\left(\Sigma_{p_{1}} X_{1}\right)^{\perp} *\left(\Sigma_{p_{2}} X_{2}\right)^{\perp}$ with orbit space isometric to $X$. Recall that given an orbit space $Y / \Gamma$ (with a stratification and assigned isotropy groups), it may not be possible to recover the $\Gamma$-action on $Y$. Here that we are able to recover the $\Gamma$-action is based on (0.1.1) (see Lemma 1.2 below) and the rigidity part of Toponogov's comparison theorem which implies that everywhere there is isometrically embedded spherical triangle; precisely, for all $p_{i}, p_{i}^{\prime} \in X_{2}$, the hinge, $\left(\left[p_{1} p_{2}\right],\left[p_{1} p_{1}^{\prime}\right], \frac{\pi}{2}\right)$ or $\left(\left[p_{2} p_{1}\right],\left[p_{2} p_{2}^{\prime}\right], \frac{\pi}{2}\right)$ bounds an isometrically embedded spherical triangle ([GM]), where $[p q]$ denotes a minimal geodesic from $p$ to $q$.

We notice that on compact manifolds of non-positive sectional curvature, the higher rank rigidity theorem (cf. [BGS], and reference within) is based on the 
geometry that at every point and in every direction, there is isometrically embedded plane.

Remark 0.5. We mention that recently, an isometric classification has been obtained by $[\mathrm{SSW}$ ] for Riemannian $n$-manifolds of sec $\geq 1$ which contains two compact totally geodesic submanifolds $X_{i}$ such that $\left|X_{1} X_{2}\right| \geq \frac{\pi}{2}$ and $\operatorname{dim}\left(X_{1}\right)+\operatorname{dim}\left(X_{2}\right)=n-2$.

Given a subset $A \subset X \in \operatorname{Alex}^{n}(1)$, let $A \geq \frac{\pi}{2}=\left\{x \in X|| x A \mid \geq \frac{\pi}{2}\right\}$ and let $A^{=\frac{\pi}{2}}=\left\{x \in X|| x a \mid=\frac{\pi}{2}, \forall a \in A\right\}$. Note that $A^{\geq \frac{\pi}{2}}$ is totally convex in $X$ (see $(1.1 .1))$.

Our last theorem asserts that the conclusion of (A2) still holds if one of $X_{i}$ has a non-empty boundary and some addition restrictions.

Theorem B. Let $X \in \operatorname{Alex}^{n}(1)$, and let $X_{1}, X_{2} \subset X$ be two compact convex subsets. Assume that $\left|X_{1} X_{2}\right| \geq \frac{\pi}{2}, \partial X_{1}=\emptyset, \partial X_{2} \neq \emptyset$, and $\operatorname{dim}\left(X_{1}\right)+\operatorname{dim}\left(X_{2}\right)=$ $n-1$. If $X_{2}^{\geq \frac{\pi}{2}}=X_{2}^{=\frac{\pi}{2}}$ and $\left(\Sigma_{p_{2}} X_{2}\right) \geq \frac{\pi}{2}=\left(\Sigma_{p_{2}} X_{2}\right)=\frac{\pi}{2}$ for all $p_{2} \in \partial X_{2}$, then $X$ is isometric to a finite quotient of join as in Theorem $A$.

In Theorem B, if $X_{2}=\left\{p_{2}\right\}$, then $X$ is either isometric to $S_{+}\left(X_{1}\right)$, or isometric to $S\left(\Sigma_{p_{2}} X\right) / \mathbb{Z}_{2}$ and $X_{1}$ is isometric to $\left(\Sigma_{p_{2}} X\right) / \mathbb{Z}_{2}$.

We now give a brief description on our approach to Theorem A.

We first give some conventions on the notations in the paper. Let $\uparrow_{x_{1}}^{x_{2}}$ denote the direction at $x_{1}$ of a given $\left[x_{1} x_{2}\right]$, let $\uparrow_{x_{1}}^{x_{2}}=\left\{\left(\uparrow_{x_{1}}^{x_{2}}\right)_{i}\right\}$ denote the collection of $\uparrow_{x_{1}}^{x_{2}}$ 's, and let $\Uparrow_{x_{1}}^{Z}=\bigcup_{z \in Z} \Uparrow_{x_{1}}^{z}$

Observe that $\left|X_{1} X_{2}\right| \geq \frac{\pi}{2}$ implies that for all $p_{i} \in X_{i},\left|p_{1} p_{2}\right|=\frac{\pi}{2}$ (see Lemma 1.3 below $)$, and thus $\Uparrow_{p_{1}}^{X_{2}} \subseteq\left(\Sigma_{p_{1}} X_{1}\right)^{\perp}$. We consider the multi-value map $f_{p_{1}}: X_{2} \rightarrow$ $\left(\Sigma_{p_{1}} X_{1}\right)^{\perp}$ defined by $f_{p_{1}}\left(p_{2}^{\prime}\right)=\Uparrow_{p_{1}}^{p_{2}^{\prime}}$. Then, $f_{p_{1}}\left(X_{2}\right)=\Uparrow_{p_{1}}^{X_{2}}$. Based on the rigidity part of Toponogov's comparison theorem, a crucial observation is:

(0.6) $f_{p_{1}}$ is a 'radial isometry', i.e., any given $\left[p_{1} p_{2}\right]$ and $\left[p_{2} p_{2}^{\prime}\right]$ bounds an embedded standard spherical triangle, whose third side, $\left[p_{1} p_{2}^{\prime}\right]$, determines $\left[\uparrow_{p_{1}}^{p_{2}} \uparrow_{p_{1}}^{p_{2}^{\prime}}\right] \subset$ $\left(\Sigma_{p_{1}} X_{1}\right)^{\perp}$ such that $f_{p_{1}}:\left[p_{2} p_{2}^{\prime}\right] \rightarrow\left[\uparrow_{p_{1}}^{p_{2}} \uparrow_{p_{1}}^{p_{2}^{\prime}}\right]$ is an isometry.

By Toponogov's comparison theorem and (0.6), $f_{p_{1}}$ is distance non-decreasing, so by induction $\operatorname{dim}\left(X_{1}\right)+\operatorname{dim}\left(X_{2}\right) \leq \operatorname{dim}\left(X_{1}\right)+\operatorname{dim}\left(\left(\Sigma_{p_{1}} X_{1}\right)^{\perp}\right) \leq n-1((\mathrm{~A} 1))$.

In the proof of (A2), a key is to show that $\Uparrow_{p_{1}}^{X_{2}}=\left(\Sigma_{p_{1}} X_{1}\right)^{\perp}$ and (and $\Uparrow_{p_{2}}^{X_{1}}=$ $\left.\left(\Sigma_{p_{2}} X_{2}\right)^{\perp}\right)$ (see Key Lemma 1.7), and it suffices to show that $\Uparrow_{p_{1}}^{X_{2}}\left(\subseteq\left(\Sigma_{p_{1}} X_{1}\right)^{\perp}\right)$ is a compact top dimensional locally convex subset without boundary. Using (0.6), at $p_{2}$ and any $\uparrow_{p_{1}}^{p_{2}} \in f_{p_{1}}\left(p_{2}\right)$, we define a natural multi-valued 'tangent map' of $f_{p_{1}}, \mathrm{D} f_{p_{1}}: \Sigma_{p_{2}} X_{2} \rightarrow \Sigma_{\uparrow_{p_{1}}}\left(\Sigma_{p_{1}} X_{1}\right)^{\perp}$, that is also distance non-decreasing. More importantly, $\mathrm{D} f_{p_{1}}$ is also a radial isometry as well, which allows us to proceed an induction to prove that $\Uparrow_{p_{1}}^{X_{2}}=\left(\Sigma_{p_{1}} X_{1}\right)^{\perp}$.

In practice, we first show that for all $p_{i} \in X_{i},\left|\Uparrow_{p_{1}}^{p_{2}}\right| \leq m<\infty$ and pairs of points at which $\left|\Uparrow_{p_{1}}^{p_{2}}\right|=m$ are dense in $X_{i}$ (see Key Lemma 1.6). The boundedness is used to show that $\mathrm{D} f_{p_{1}}$ is a radial isometry and $\left|\mathrm{D} f_{p_{1}}(\xi)\right|\left(\xi \in \Sigma_{p_{2}} X_{2}\right)$ has a similar boundedness.

Once $\Uparrow_{p_{1}}^{X_{2}}=\left(\Sigma_{p_{1}} X_{1}\right)^{\perp}$ is established, fixing $p_{i}$ with $\left|\Uparrow_{p_{1}}^{p_{2}}\right|=m$ we can construct a finite group $\Gamma_{2}$ acting isometrically on $\left(\Sigma_{p_{1}} X_{1}\right)^{\perp}$ such that $\Gamma_{2}\left(\uparrow p_{p_{1}}^{p_{2}}\right)=\uparrow_{p_{1}}^{p_{2}}$, and $\left(\Sigma_{p_{1}} X_{1}\right)^{\perp} / \Gamma_{2}$ is isometric to $X_{2}$. Given $\uparrow_{p_{2}}^{p_{1}}$ and $\uparrow_{p_{2}}^{p_{1}} \neq\left(\uparrow_{p_{2}}^{p_{1}}\right)_{i} \in \uparrow_{p_{2}}^{p_{1}}$, a geodesic 
jointing the two points determines a geodesic loop $\gamma_{i} \subset X_{1}$ at $p_{1}$. For each $\gamma_{i}$, we define an isometry on $\left(\Sigma_{p_{1}} X_{1}\right)^{\perp}$ via a successive 'parallel transport' along $\gamma_{i}$ piece by piece. The independence of the isometry on the choice of $\gamma_{i}$ relies on the fact that isometrically embedded spherical triangles exist everywhere (see Remark 0.4).

Similarly, we can construct a finite group $\Gamma_{1}$ acting isometrically on $\left(\Sigma_{p_{2}} X_{2}\right)^{\perp}$. And from the construction of $\Gamma_{i}$, it is not hard to show that $\Gamma_{1}$ is isomorphic to $\Gamma_{2}$. Consequently, we obtain an isometric $\Gamma$-action on $\left(\Sigma_{p_{1}} X_{1}\right)^{\perp} *\left(\Sigma_{p_{2}} X_{2}\right)^{\perp}$, and it remains to show that $X$ is isometric to the orbit space. Note that a consequence of $\Uparrow_{p_{1}}^{X_{2}}=\left(\Sigma_{p_{1}} X_{1}\right)^{\perp}$ is that for any $x \in X-\left(X_{1} \cup X_{2}\right)$, there are unique $p_{i} \in X_{i}$ and a minimal geodesic jointing $p_{1}$ with $p_{2}$ such that $x \in\left[p_{1} p_{2}\right]$. This property guarantees that the natural map from $\left[\left(\Sigma_{p_{1}} X_{1}\right)^{\perp} *\left(\Sigma_{p_{2}} X_{2}\right)^{\perp}\right] / \Gamma$ to $X$ is a bijection, which preserves the distance (this also relies on the rigidity part of Toponogov's comparison theorem).

\section{The Geometry of $\frac{\pi}{2}$-Apart Convex Subsets}

In the proof of Theorem A, we will often apply Toponogov's comparison theorem, in particular its rigidity part in (1.1.3). Recall that $|p q|$ (resp. $[p q]$ ) denotes the distance (resp. a minimal geodesic) between $p$ and $q$.

Theorem 1.1 ([BGP]). Let $X \in$ Alex $^{n}(\kappa)$, and let $\mathbb{S}_{\kappa}^{2}$ be the complete, simply connected 2-manifold of curvature $\kappa$.

(1.1.1) To any $p \in X$ and $[q r] \subset X$, we associate $\tilde{p}$ and $a[\tilde{q} \tilde{r}]$ in $\mathbb{S}_{\kappa}^{2}$ with $|\tilde{p} \tilde{q}|=$ $|p q|,|\tilde{p} \tilde{r}|=|p r|$ and $|\tilde{r} \tilde{q}|=|r q|$. Then for any $s \in[q r]$ and $\tilde{s} \in[\tilde{q} \tilde{r}]$ with $|q s|=|\tilde{q} \tilde{s}|$, we have that $|p s| \geq|\tilde{p} \tilde{s}|$.

(1.1.2) To any $[q p]$ and $[q r]$ in $X$, we associate $[\tilde{q} \tilde{p}]$ and $[\tilde{q} \tilde{r}]$ in $\mathbb{S}_{\kappa}^{2}$ with $|\tilde{q} \tilde{p}|=|q p|$, $|\tilde{q} \tilde{r}|=|q r|$ and $\angle \tilde{p} \tilde{q} \tilde{r}=\angle p q r$. Then we have that $|\tilde{p} \tilde{r}| \geq|p r|$.

(1.1.3) ([GM]) If equality in (1.1.2) (resp. in (1.1.1) for some interior point $s$ in $[q r]$ ) holds, then there exists a $[p r]$ (resp. $[q p]$ and $[p r]$ ) such that $[q p],[q r]$ and $[p r]$ bounds a surface which is convex and can be isometrically embedded into $\mathbb{S}_{\kappa}^{2}$.

We first observe the following fact on $X_{1}$ and $X_{2}$ in Theorem A.

Lemma 1.2. Let $X \in$ Alex $^{n}(1)$. Assume that $X_{1}, X_{2}$ are compact convex subsets such that $\left|X_{1} X_{2}\right| \geq \frac{\pi}{2}$. If $\partial X_{1}=\emptyset$ (or $\partial X_{2}=\emptyset$ ), then $\left|x_{1} x_{2}\right|=\frac{\pi}{2}$ for all $x_{i} \in X_{i}$.

Lemma 1.2 is a consequence of the following lemma.

Lemma 1.3 ([Ya]). Let $X \in$ Alex $^{n}(1)$, and let $A$ be a compact (locally) convex subset in $X$. If $\partial A=\emptyset$, then $A^{\geq \frac{\pi}{2}}=A^{=\frac{\pi}{2}}$.

Note that using (1.1.2), one can derive Lemma 1.3 by induction on $n$.

From now on, we let $X_{1}, X_{2} \subset X \in \operatorname{Alex}^{n}(1)$ be compact convex subsets with

$$
\left|x_{1} x_{2}\right|=\frac{\pi}{2} \text { for all } x_{i} \in X_{i}
$$

Let $X_{i}^{\circ}$ denote the interior part of $X_{i}^{5}$. For $p_{1} \in X_{1}^{\circ}$ and any $p_{2} \in X_{2}$ and any $\left[p_{1} p_{2}\right]$, it is easy to see that

$$
\uparrow_{p_{1}}^{p_{2}} \in\left(\Sigma_{p_{1}} X_{1}\right)^{\perp}
$$

${ }^{5}$ At any $p \in X^{\circ}$ and $\partial X, \Sigma_{p} X$ has empty and non-empty boundary respectively ([BGP]). 
Since $\left|x_{1} p_{2}\right|=\frac{\pi}{2}$ for all $x_{1} \in X_{1}$, by the first variation formula ([BGP]) it follows that $\uparrow_{p_{1}}^{p_{2}} \in\left(\Sigma_{p_{1}} X_{1}\right) \geq \frac{\pi}{2}$. Then (1.1) follows from Lemma 1.3 (note that $p_{1} \in X_{1}^{\circ}$ and $X_{1}$ is convex, thus $\Sigma_{p_{1}} X_{1}$ is a convex subset with an empty boundary). Similarly, for any $\left[p_{1} p_{2}\right]$ with $p_{1} \in X_{1}$ and $p_{2} \in X_{2}^{\circ}$, we have that

$$
\uparrow_{p_{2}}^{p_{1}} \in\left(\Sigma_{p_{2}} X_{2}\right)^{\perp}
$$

By (1.2) and (1.1.3), we have the following property.

Lemma 1.4. For $p_{1} \in X_{1}, p_{2} \in X_{2}^{\circ}$, given $\left[p_{1} p_{2}\right]$ and $\left[p_{2} p_{2}^{\prime}\right] \subset X_{2}$, there exists $a\left[p_{1} p_{2}^{\prime}\right]$ such that $\left[p_{1} p_{2}\right],\left[p_{2} p_{2}^{\prime}\right]$ and $\left[p_{1} p_{2}^{\prime}\right]$ bound a convex surface which can be embedded isometrically into the unit sphere $\mathbb{S}^{2}$.

We make a convention that a convex surface in $X$ as in Lemma 1.4 is called a convex spherical surface. And in the rest, if $\operatorname{dim}\left(X_{i}\right)=0$, we let $X_{i}^{\circ}=X_{i}$.

Now, we assume that $\operatorname{dim}\left(X_{2}\right)>0$, and let $p_{2} \in X_{2}^{\circ}$ and $p_{1} \in X_{1}^{\circ}$. Let $Q\left(p_{2}\right)$ denote the 'cut locus' of $p_{2}$ in $X_{2}$, i.e., $Q\left(p_{2}\right)=\left\{x \in X,\left[p_{2} x\right]\right.$ is a maximal minimal geodesic $\}$. For a fixed $\left[p_{1} p_{2}\right]$, given $\left[p_{2} p_{2}^{\prime}\right]$ with $p_{2}^{\prime} \in Q\left(p_{2}\right)$, by $(1.2)$ and Lemma 1.4 there is a convex spherical surface $S \supset\left[p_{1} p_{2}\right],\left[p_{2} p_{2}^{\prime}\right]$ (note that the convex spherical surface may not be unique). Let $\left.c(t)\right|_{t \in[0,1]}=\left[p_{2} p_{2}^{\prime}\right]$ with $c(0)=p_{2}$ and $c(1)=p_{2}^{\prime}$, and $\left[p_{1} c(t)\right] \subset S$. We define a map

$$
f_{\left[p_{1} p_{2}\right]}(c(t))=\uparrow_{p_{1}}^{c(t)} \in\left(\Sigma_{p_{1}} X_{1}\right)^{\perp}(\text { see }(1.1))
$$

Note that $c(t)$ with $0<t<1$ is in the unique maximal minimal geodesic $\left[p_{2} p_{2}^{\prime}\right]$, so $f_{\left[p_{1} p_{2}\right]}$ is well-defined on $X_{2}-Q\left(p_{2}\right)$. But it is possible that there are more than one minimal geodesic between $p_{2}$ and $p_{2}^{\prime}$. Anyway, by choosing an arbitrary $f_{\left[p_{1} p_{2}\right]}\left(p_{2}^{\prime}\right)$ for $p_{2}^{\prime} \in Q$, we can define a map

$$
f_{\left[p_{1} p_{2}\right]}: X_{2} \rightarrow\left(\Sigma_{p_{1}} X_{1}\right)^{\perp}
$$

From the definition, it is clear that for any $p_{2}^{\prime} \in X_{2}$,

$$
\left|f_{\left[p_{1} p_{2}\right]}\left(p_{2}\right) f_{\left[p_{1} p_{2}\right]}\left(p_{2}^{\prime}\right)\right|=\left|p_{2} p_{2}^{\prime}\right|
$$

and that if there is a unique minimal geodesic between $p_{2}$ and $p_{2}^{\prime}$, then $f_{\left[p_{1} p_{2}\right]}\left(\left[p_{2} p_{2}^{\prime}\right]\right)$ is a minimal geodesic in $\left(\Sigma_{p_{1}} X_{1}\right)^{\perp}$. Consequently, $f_{\left[p_{1} p_{2}\right]}$ naturally induces a tangent map

$$
\mathrm{D} f_{\left[p_{1} p_{2}\right]}: \Sigma_{p_{2}} X_{2} \longrightarrow \Sigma_{\uparrow_{p_{1}}^{p_{2}}}\left(\Sigma_{p_{1}} X_{1}\right)^{\perp}
$$

On the other hand, by (1.1.2) we have that

$$
\left|f_{\left[p_{1} p_{2}\right]}\left(p_{2}^{\prime}\right) f_{\left[p_{1} p_{2}\right]}\left(p_{2}^{\prime \prime}\right)\right| \geq\left|p_{2}^{\prime} p_{2}^{\prime \prime}\right|
$$

for all $p_{2}^{\prime}, p_{2}^{\prime \prime} \in X_{2}$.

From the above discussion, we conclude the following.

Proposition 1.5. (1.5.1) $f_{\left[p_{1} p_{2}\right]}$ is distance non-decreasing; and $f_{\left[p_{1} p_{2}\right]}$ maps a $\left(n_{2}, \delta\right)$-strainer at $p_{2}$ to a $\left(n_{2}, \delta\right)$-strainer at $\uparrow_{p_{1}}^{p_{2}}$, and thus $\operatorname{dim}\left(X_{2}\right) \leq \operatorname{dim}\left(\left(\Sigma_{p_{1}} X_{1}\right)^{\perp}\right)$. (1.5.2) $\mathrm{D} f_{\left[p_{1} p_{2}\right]}$ is also a distance non-decreasing map. 
Proof. By (1.4), $f_{\left[p_{1} p_{2}\right]}$ is distance non-decreasing. Then by (1.3) and (1.1.2), $f_{\left[p_{1} p_{2}\right]}$ preserves an $\left(n_{2}, \delta\right)$-strainer (see [BGP] for the definition of the $(n, \delta)$-strainer), and $\mathrm{D} f_{\left[p_{1} p_{2}\right]}$ is distance non-decreasing.

The observation that both $f_{\left[p_{1} p_{2}\right]}$ and $\mathrm{D} f_{\left[p_{1} p_{2}\right]}$ are distance nondecreasing maps will play a key rule in the proof of Theorem A.

Based on the geometry of two $\frac{\pi}{2}$-part convex subsets in Lemma 1.2 and 1.4, and properties of $f_{\left[p_{1} p_{2}\right]}$ and $\mathrm{D} f_{\left[p_{1} p_{2}\right]}$ in Proposition 1.5, we will establish the following key properties that are used in the proof of Theorem A (see the outline of the proof at the end of Introduction).

Let $\lambda_{x_{1} x_{2}}$ be the number of minimal geodesics between $x_{1}$ and $x_{2}$.

Key Lemma 1.6. Let $X \in$ Alex $^{n}(1)$, and let $X_{1}$ and $X_{2}$ be its compact convex subsets with $\left|x_{1} x_{2}\right|=\frac{\pi}{2}$ for all $x_{i} \in X_{i}$ and $\operatorname{dim}\left(X_{1}\right)+\operatorname{dim}\left(X_{2}\right)=n-1$. Then $\lambda_{p_{1} p_{2}}$ has a maximum $m$ for $p_{i} \in X_{i}^{\circ}$; moreover, $X_{i}^{m} \triangleq\left\{p \in X_{i}^{\circ} \mid \exists q \in X_{j}^{\circ}(j \neq\right.$ i) s.t. $\left.\lambda_{p q}=m\right\}$ is open and dense in $X_{i}^{\circ}$, and $\lambda_{p_{1} p_{2}}=m$ for all $p_{i} \in X_{i}^{m}$.

Key Lemma 1.7. Let the assumptions be as in Key Lemma 1.6. For any $\left[p_{1} p_{2}\right]$ with $p_{i} \in X_{i}^{\circ}$, we have that $\uparrow_{p_{1}}^{p_{2}} \in\left(\left(\Sigma_{p_{1}} X_{1}\right)^{\perp}\right)^{\circ}$. In particular, if $\partial X_{2}=\emptyset$, then $\partial\left(\Sigma_{p_{1}} X_{1}\right)^{\perp}=\emptyset$, and $\left(\Sigma_{p_{1}} X_{1}\right)^{\perp}=\Uparrow_{p_{1}}^{X_{2}}$.

We conclude this section with an application of Proposition 1.5, which together with Lemma 1.2 implies (A1).

Theorem 1.8. Let $X \in \operatorname{Alex}^{n}(1)$, and let $X_{1}$ and $X_{2}$ be its two compact convex subsets with $\left|x_{1} x_{2}\right|=\frac{\pi}{2}$ for all $x_{i} \in X_{i}$. Then $\operatorname{dim}\left(X_{1}\right)+\operatorname{dim}\left(X_{2}\right) \leq n-1$.

Proof. We observe that Theorem 1.8 holds if $n=1$, and proceed by induction on $n$. For $p_{1} \in X_{1}^{\circ}$, the following subsets of $\Sigma_{p_{1}} X$ are convex:

$$
\Sigma_{p_{1}} X_{1}, \quad\left(\Sigma_{p_{1}} X_{1}\right)^{\perp}\left(=\left(\Sigma_{p_{1}} X_{1}\right)^{=\frac{\pi}{2}}\right) .
$$

(Here, if $\operatorname{dim}\left(X_{1}\right)=0$, then $\Sigma_{p_{1}} X_{1}=\emptyset$ and $\operatorname{dim}\left(\Sigma_{p_{1}} X_{1}\right)=-1$, and $\left(\Sigma_{p_{1}} X_{1}\right)^{\perp}=$ $\Sigma_{p_{1}} X$. Note that $\Sigma_{p_{1}} X_{1}$ is convex and has an empty boundary because $p_{1} \in X_{1}^{\circ}$ and $X_{1}$ is convex, so by Lemma $1.3\left(\Sigma_{p_{1}} X_{1}\right)^{=\frac{\pi}{2}}=\left(\Sigma_{p_{1}} X_{1}\right) \geq \frac{\pi}{2}$, which is convex by (1.1.1).) Since $\Sigma_{p_{1}} X \in \operatorname{Alex}^{n-1}(1)$, by induction we have that

$$
\operatorname{dim}\left(\Sigma_{p_{1}} X_{1}\right)+\operatorname{dim}\left(\left(\Sigma_{p_{1}} X_{1}\right)^{\perp}\right) \leq \operatorname{dim}\left(\Sigma_{p_{1}} X\right)-1=n-2
$$

which implies

$$
\operatorname{dim}\left(X_{1}\right)+\operatorname{dim}\left(\left(\Sigma_{p_{1}} X_{1}\right)^{\perp}\right) \leq n-1 .
$$

On the other hand, by (1.5.1) we have that

$$
\operatorname{dim}\left(X_{2}\right) \leq \operatorname{dim}\left(\left(\Sigma_{p_{1}} X_{1}\right)^{\perp}\right)
$$

Hence, $\operatorname{dim}\left(X_{1}\right)+\operatorname{dim}\left(X_{2}\right) \leq n-1$.

Remark 1.9. From the above proof, it is clear that " $\operatorname{dim}\left(X_{1}\right)+\operatorname{dim}\left(X_{2}\right)=n-1 "$ implies that $\operatorname{dim}\left(\left(\Sigma_{p_{i}} X_{i}\right)^{\perp}\right)=\operatorname{dim}\left(X_{j}\right)(j \neq i)$ for $p_{i} \in X_{i}^{\circ}$, and that $\left(\Sigma_{p_{i}} X_{i}\right)^{\perp}$ consists of either a point or two points with distance $\pi$ if $\operatorname{dim}\left(\left(\Sigma_{p_{i}} X_{i}\right)^{\perp}\right)=0$. 


\section{Proof of Key Lemma 1.6}

We first point out that Key Lemma 1.6 is obvious if $X_{1}=\left\{p_{1}, p_{1}^{\prime}\right\}$ (or $X_{2}=$ $\left.\left\{p_{2}, p_{2}^{\prime}\right\}\right)$. It is our convention that $\left|p_{1} p_{1}^{\prime}\right|=\pi$, and thus $X=X_{1} * N$ and $X_{2} \subseteq$ $N \in \operatorname{Alex}^{n-1}(1)$. Hence, $\lambda_{p_{1} p_{2}}=1$.

In the following proof, we need only to consider two cases: $X_{1}$ is a point, and $\operatorname{dim}\left(X_{i}\right)>0, i=1,2$. Let's first bound $\lambda_{p_{1} p_{2}}$ in Key Lemma 1.6.

Lemma 2.1. (2.1.1) If $X_{1}=\left\{p_{1}\right\}$, then $\lambda_{p_{1} p_{2}} \leq 2$ for any $p_{2} \in X_{2}^{\circ}$.

(2.1.2) If $\operatorname{dim}\left(X_{i}\right)>0$ for $i=1$ and 2 , then there exists a positive number $m$ such that $\lambda_{p_{1} p_{2}} \leq m$ for any $p_{i} \in X_{i}^{\circ}$.

Proof. (2.1.1) For any $p_{2} \in X_{2}^{\circ}$, by Remark $1.9 \operatorname{dim}\left(\left(\Sigma_{p_{2}} X_{2}\right)^{\perp}\right)=0$ (note that $\left.\operatorname{dim}\left(X_{2}\right)=n-1\right)$, and thus $\left(\Sigma_{p_{2}} X_{2}\right)^{\perp}$ is either a point or two points with distance $\pi$. Since $\uparrow_{p_{2}}^{p_{1}} \in\left(\Sigma_{p_{2}} X_{2}\right)^{\perp}$ for any $\left[p_{1} p_{2}\right]$ (see (1.2)), we have that $\lambda_{p_{1} p_{2}} \leq 2$.

(2.1.2) By (2.1.1), we are able to apply the inductive argument starting with $\operatorname{dim}\left(X_{1}\right)=0$. Let $n_{i}=\operatorname{dim}\left(X_{i}\right)$.

We first give the proof for a special case where $p_{i} \in X_{i}^{\circ}$ is an $\left(n_{i}, \delta\right)$-strained point (i.e. a point with an $\left(n_{i}, \delta\right)$-strainer).

Firstly, under an additional assumption that there is $\left(n_{i}, \delta\right)$-strained point $q_{i} \in$ $X_{i}$ such that $\lambda_{q_{1} q_{2}}<\infty$, we prove that $\lambda_{p_{1} p_{2}}$ has a maximum $m$. If this is not true, then there is a sequence of $\left(n_{i}, \delta\right)$-strained points $p_{i}^{k} \in X_{i}$ such that $\lim _{k \rightarrow \infty} \lambda_{p_{1}^{k} p_{2}^{k}}=\infty$. Now we consider $\lambda_{q_{1} p_{2}^{k}}$. If $\lim _{k \rightarrow \infty} \lambda_{q_{1} p_{2}^{k}}<\infty$ (resp. $\left.\lim _{k \rightarrow \infty} \lambda_{q_{1} p_{2}^{k}}=\infty\right)$ passing a subsequence, then by Lemma 1.4 there is $\left[q_{1} p_{2}^{k}\right]$ and $\left[q_{1} p_{1}^{k}\right]$ (resp. $\left[q_{2} q_{1}\right]_{k}$ and $\left[q_{2} p_{2}^{k}\right]$, where $\left[q_{2} q_{1}\right]_{k}$ is some minimal geodesic between $q_{2}$ and $\left.q_{1}\right)$ such that

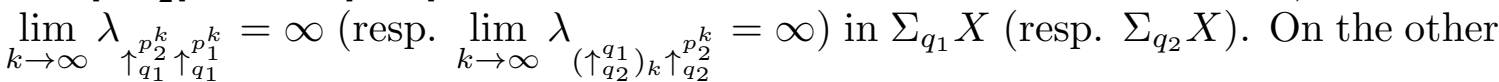
hand, according to Remark 1.9, $\operatorname{dim}\left(\left(\Sigma_{q_{1}} X_{1}\right)^{\perp}\right)=n_{2}$ and $\operatorname{dim}\left(\left(\Sigma_{q_{2}} X_{2}\right)^{\perp}\right)=n_{1}$; and by Proposition 1.5, $\uparrow_{q_{1}}^{p_{2}^{k}}$ and $\left(\uparrow_{q_{2}}^{p_{1}}\right)_{k}$ are $\left(n_{2}, \delta\right)$ - and $\left(n_{1}, \delta\right)$-strained points in $\left(\Sigma_{q_{1}} X_{1}\right)^{\perp}$ and $\left(\Sigma_{q_{2}} X_{2}\right)^{\perp}$ respectively. This implies that $\uparrow_{q_{1}}^{p_{2}^{k}} \in\left(\left(\Sigma_{q_{1}} X_{1}\right)^{\perp}\right)^{\circ}$ and $\left(\uparrow_{q_{2}}^{q_{1}}\right)_{k} \in\left(\left(\Sigma_{q_{2}} X_{2}\right)^{\perp}\right)^{\circ}$. Moreover, $\uparrow_{q_{i}}^{q_{i}^{k}} \in\left(\Sigma_{q_{i}} X_{i}\right)^{\circ}$ because $\Sigma_{q_{i}} X_{i}$ has an empty boundary. Then, by the inductive assumption on $\Sigma_{q_{1}} X$ (resp. $\Sigma_{q_{2}} X$ ), we can conclude that $\lambda_{\uparrow_{q_{1} \uparrow_{q_{1}}^{p_{1}^{k}}}^{p_{1}^{k}}}$ (resp. $\lambda_{\left(\uparrow_{\left.q_{2}\right)_{k} \uparrow_{q_{2}}^{q_{1}}}^{p_{2}^{k}}\right)}$ have an upper bound for all $k$, a contradiction.

Secondly, we verify the above additional assumption, i.e., there is $\left(n_{i}, \delta\right)$-strained point $q_{i} \in X_{i}$ such that $\lambda_{q_{1} q_{2}}<\infty$. If not true, then for any $\left(n_{i}, \delta\right)$-strained point $p_{i} \in X_{i}, \lambda_{p_{1} p_{2}}=\infty$. Note that " $\lambda_{p_{1} p_{2}}=\infty$ " implies that there are minimal geodesics $\left[p_{1} p_{2}\right]$ and $\left\{\left[p_{1} p_{2}\right]_{j}\right\}_{j=1}^{\infty}$ between $p_{1}$ and $p_{2}$ such that

$$
\lim _{j \rightarrow \infty}\left[p_{1} p_{2}\right]_{j}=\left[p_{1} p_{2}\right]\left(\text { so } \lim _{j \rightarrow \infty}\left(\uparrow p_{p_{1}}^{p_{2}}\right)_{j}=\uparrow_{p_{1}}^{p_{2}}\right) .
$$

Now we consider the maps $f_{\left[p_{1} p_{2}\right]}$ and $\mathrm{D} f_{\left[p_{1} p_{2}\right]}$ (defined in Section 1). For convenience, we let $\zeta_{j}$ and $\zeta$ denote $\left(\uparrow_{p_{1}}^{p_{2}}\right)_{j}$ and $\uparrow_{p_{1}}$ respectively. By Proposition 1.5, $f_{\left[p_{1} p_{2}\right]}$ maps an $\left(n_{2}, \delta\right)$-strainer at $p_{2}$ in $X_{2}$ to an $\left(n_{2}, \delta\right)$-strainer at $\zeta$ in $\left(\Sigma_{p_{1}} X_{1}\right)^{\perp}$. On the other hand, we have $\operatorname{dim}\left(\left(\Sigma_{p_{1}} X_{1}\right)^{\perp}\right)=n_{2}$ (see Remark 1.9). It then follows that both $\Sigma_{p_{2}} X_{2}$ and $\Sigma_{\zeta}\left(\Sigma_{p_{1}} X_{1}\right)^{\perp}$ are $\chi(\delta)$-isometric to the unit sphere $\mathbb{S}^{n_{2}-1}$, where $\chi(\delta) \rightarrow 0$ as $\delta \rightarrow 0$ (ref. [BGP]). Together with that $\mathrm{D} f_{\left[p_{1} p_{2}\right]}$ is distance nondecreasing (see Proposition 1.5), this implies that for any $\eta \in \Sigma_{\zeta}\left(\Sigma_{p_{1}} X_{1}\right)^{\perp}$ there exists $\xi_{\eta} \in \Sigma_{p_{2}} X_{2}$ such that $\left|\mathrm{D} f_{\left[p_{1} p_{2}\right]}\left(\xi_{\eta}\right) \eta\right|<\chi(\delta)$. Moreover, we can assume 
that there is a $\left[p_{2} q_{\eta}\right]$ in $X_{2}$ such that $\xi_{\eta}=\uparrow_{p_{2}}^{q_{\eta}}$, so $\mathrm{D} f_{\left[p_{1} p_{2}\right]}\left(\xi_{\eta}\right)$ is the direction of $f_{\left[p_{1} p_{2}\right]}\left(\left[p_{2} q_{\eta}\right]\right)$ at $\zeta$. Since $\lim _{j \rightarrow \infty} \zeta_{j}=\zeta$, we assume that $\uparrow_{\zeta}^{\zeta_{j}}$ converges to some $\bar{\eta} \in \Sigma_{\zeta}\left(\Sigma_{p_{1}} X_{1}\right)^{\perp}$ passing to a subsequence. Hence, for sufficiently large $j_{0}$, we can assume that $\left|\mathrm{D} f_{\left[p_{1} p_{2}\right]}\left(\xi_{\bar{\eta}}\right) \uparrow_{\zeta}^{\zeta_{j_{0}}}\right|<2 \chi(\delta)$, and select $\zeta_{0} \triangleq f_{\left[p_{1} p_{2}\right]}\left(p_{2}^{0}\right)$ with $p_{2}^{0} \in\left[p_{2} q_{\bar{\eta}}\right]$ such that $\left|\zeta \zeta_{0}\right|=\left|\zeta \zeta_{j_{0}}\right|$ (note that $\left|\zeta \zeta_{0}\right|=\left|p_{2} p_{2}^{0}\right|$ ). By applying (1.1.2) on the hinge formed by $\left[\zeta \zeta_{0}\right]$ and $\left[\zeta \zeta_{j_{0}}\right]$, we conclude that

$$
\left|\zeta_{j_{0}} \zeta_{0}\right|<2 \chi(\delta)\left|\zeta \zeta_{0}\right|
$$

However, by applying (1.1.2) on the hinge formed by $\left[p_{1} p_{2}\right]_{j_{0}}$ and $\left[p_{1} p_{2}^{0}\right]$ (with $\left.\uparrow p_{2}^{p_{2}^{0}}=\zeta_{0}\right)$, we conclude that

$$
\left|\zeta_{j_{0}} \zeta_{0}\right| \geq\left|p_{2} p_{2}^{0}\right|=\left|\zeta \zeta_{0}\right|
$$

a contradiction. By now, the proof for the special case is complete.

Next, we will prove that $\lambda_{p_{1} p_{2}} \leq m$ when one of $p_{i}$ is $\left(n_{i}, \delta\right)$-strained point, say $p_{1}$. If $\lambda_{p_{1} p_{2}}>m$, we select minimal geodesics $\left\{\left[p_{1} p_{2}\right]_{k}\right\}_{k=1}^{m+1}$, and let

$$
\varepsilon=\min _{1 \leq i \neq j \leq m+1}\left\{\left|\left(\uparrow p_{p_{1}}^{p_{2}}\right)_{i}\left(\uparrow_{p_{1}}^{p_{2}}\right)_{j}\right|\right\}
$$

Then by Lemma 2.2 below, we get that $\lambda_{p_{1} p_{2}^{\prime}} \geq m+1$ for any $p_{2}^{\prime} \in B\left(p_{2}, \frac{\varepsilon}{2}\right) \cap X_{2}$. However, there must be an $\left(n_{2}, \delta\right)$-strained point $p_{2}^{\prime}$ in $B\left(p_{2}, \frac{\varepsilon}{2}\right) \cap X_{2}$ (recall that, for any $\nu>0$, there are $\left(n_{2}, \delta\right)$-strained points in $\left.B\left(p_{2}, \nu\right) \cap X_{2}([\mathrm{BGP}])\right)$, so $\lambda_{p_{1} p_{2}^{\prime}} \leq m$ by the above special case, which contradicts $\lambda_{p_{1} p_{2}^{\prime}} \geq m+1$.

Finally, for any $p_{i} \in X_{i}^{\circ}$, by repeating the argument in the previous case we conclude that $\lambda_{p_{1} p_{2}} \leq \lambda_{p_{1} p_{2}^{\prime}} \leq m$, where $p_{2}^{\prime}$ is $\left(n_{2}, \delta\right)$-strained point close to $p_{2}$.

Lemma 2.2. Let $p_{1} \in X_{1}$ and $p_{2} \in X_{2}^{\circ}$, and let $\left[p_{1} p_{2}\right]_{1}, \cdots,\left[p_{1} p_{2}\right]_{k}$ be minimal

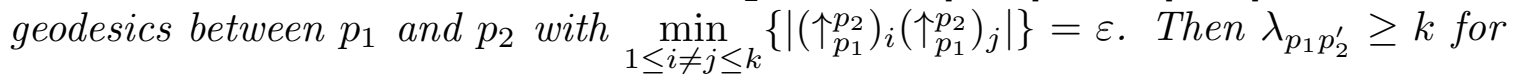
any $p_{2}^{\prime} \in B\left(p_{2}, \frac{\varepsilon}{2}\right) \cap X_{2}$.

Proof. We will derive a contradiction by assuming that $\lambda_{p_{1} p_{2}^{\prime}}<k$. By Lemma 1.4 , for any $\left[p_{1} p_{2}\right]_{j}$ there is a $\left[p_{1} p_{2}^{\prime}\right]$ such that $\left|\left(\uparrow_{p_{1}}^{p_{2}}\right)_{j} \uparrow p_{2}^{p_{2}^{\prime}}\right|=\left|p_{2} p_{2}^{\prime}\right|$. Hence, if $\lambda_{p_{1} p_{2}^{\prime}}<k$, there must be two $\left[p_{1} p_{2}\right]_{j}$, say $\left[p_{1} p_{2}\right]_{1}$ and $\left[p_{1} p_{2}\right]_{2}$, and one $\left[p_{1} p_{2}^{\prime}\right]$ such that $\left|\left(\uparrow p_{p_{1}}\right)_{j} \uparrow p_{2}^{p_{2}^{\prime}}\right|=\left|p_{2} p_{2}^{\prime}\right|(j=1,2)$. It then follows that

$$
\left|\left(\uparrow_{p_{1}}^{p_{2}}\right)_{1}\left(\uparrow_{p_{1}}^{p_{2}}\right)_{2}\right| \leq 2\left|p_{2} p_{2}^{\prime}\right|<\varepsilon
$$

which contradicts " $\min _{1 \leq i \neq j \leq k}\left\{\left|\left(\uparrow_{p_{1}}^{p_{2}}\right)_{i}\left(\uparrow_{p_{1}}^{p_{2}}\right)_{j}\right|\right\}=\varepsilon "$.

Based on Lemma 2.1, we will explore the local join structure on $X$, which will be used to show that $X_{i}^{m}$ is open and dense (the latter part of Key Lemma 1.6).

Lemma 2.3. Suppose that the function $\lambda: X_{1} \times X_{2} \rightarrow \mathbb{N}^{+}$defined by $\lambda(p, q)=$ $\lambda_{p q}$ attains a local maximum $m_{l}$ around $\left(p_{1}, p_{2}\right)$ with $p_{i} \in X_{i}^{\circ}$. Then there are neighborhoods $U_{i} \subset X_{i}^{\circ}$ of $p_{i}$ such that

(2.3.1) $\lambda_{p_{1}^{\prime} p_{2}^{\prime}}=m_{l}$ for any $p_{1}^{\prime} \in U_{1}$ and $p_{2}^{\prime} \in U_{2}$; 
(2.3.2) for any $\left[p_{1} p_{2}\right]$, both $f_{\left[p_{1} p_{2}\right]}: U_{2} \longrightarrow\left(\Sigma_{p_{1}} X_{1}\right)^{\perp}$ and $f_{\left[p_{2} p_{1}\right]}: U_{1} \longrightarrow\left(\Sigma_{p_{2}} X_{2}\right)^{\perp}$ are isometrical embedding;

(2.3.3) $U_{1} * U_{2}$ can be embedded isometrically into $X$ around any $\left[p_{1} p_{2}\right]$.

Proof. Let

$$
\varepsilon=\min _{1 \leq i \neq j \leq m_{l}}\left\{\left|\left(\uparrow_{p_{2}}^{p_{1}}\right)_{i}\left(\uparrow_{p_{2}}^{p_{1}}\right)_{j}\right|,\left|\left(\uparrow_{p_{1}}^{p_{2}}\right)_{i}\left(\uparrow_{p_{1}}^{p_{2}}\right)_{j}\right|\right\}
$$

where $\left\{\left(\uparrow_{p_{2}}^{p_{1}}\right)_{i}\right\}$ (resp. $\left.\left\{\left(\uparrow_{p_{1}}^{p_{2}}\right)_{j}\right\}\right)$ are all directions from $p_{2}$ to $p_{1}$ (resp. from $p_{1}$ to $p_{2}$ ). By Lemma 2.2, for any $p_{2}^{\prime} \in B\left(p_{2}, \frac{\varepsilon}{2}\right) \cap X_{2}$ and $p_{1}^{\prime} \in B\left(p_{1}, \frac{\varepsilon}{2}\right) \cap X_{1}$, we have that $\lambda_{p_{1} p_{2}^{\prime}} \geq m_{l}$ and $\lambda_{p_{2} p_{1}^{\prime}} \geq m_{l}$. On the other hand, there are neighborhoods $V_{i} \subset X_{i}$ of $p_{i}$ such that $\left.\lambda\right|_{V_{1} \times V_{2}} \leq m_{l}$. It then follows that, for any $p_{i}^{\prime} \in V_{i} \cap B\left(p_{i}, \frac{\varepsilon}{2}\right)$,

$$
\lambda_{p_{1} p_{2}^{\prime}}=\lambda_{p_{1}^{\prime} p_{2}}=m_{l}
$$

and from the proof of Lemma 2.2 it is easy to see that, for any fixed $\left[p_{1} p_{2}\right]$,

$$
\exists !\left[p_{1} p_{2}^{\prime}\right] \text { s.t. }\left|\uparrow_{p_{1}}^{p_{2}} \uparrow_{p_{1}}^{p_{2}^{\prime}}\right|=\left|p_{2} p_{2}^{\prime}\right| \text { and } \exists !\left[p_{2} p_{1}^{\prime}\right] \text { s.t. }\left|\uparrow_{p_{2}}^{p_{1}} \uparrow_{p_{2}}^{p_{1}^{\prime}}\right|=\left|p_{1} p_{1}^{\prime}\right| \text {. }
$$

Due to the property of the limit angle (see 2.8.1 in [BGP]), for sufficiently small convex neighborhood $U_{i} \subset V_{i} \cap B\left(p_{i}, \frac{\varepsilon}{2}\right) \cap X_{i}^{\circ}$ of $p_{i}$, we have that

$$
\begin{array}{r}
\min _{1 \leq i \neq j \leq m_{l}}\left\{\left|\left(\uparrow_{p_{1}}^{p_{2}^{\prime}}\right)_{i}\left(\uparrow_{p_{1}}^{p_{2}^{\prime}}\right)_{j}\right|,\left|\left(\uparrow_{p_{2}^{\prime}}^{p_{1}}\right)_{i}\left(\uparrow_{p_{2}^{\prime}}^{p_{1}}\right)_{j}\right|\right\}>\frac{\varepsilon}{2} \text { for any } p_{2}^{\prime} \in U_{2}, \\
\min _{1 \leq i \neq j \leq m_{l}}\left\{\left|\left(\uparrow_{p_{2}}^{p_{1}^{\prime}}\right)_{i}\left(\uparrow_{p_{2}}^{p_{1}^{\prime}}\right)_{j}\right|,\left|\left(\uparrow_{p_{1}^{\prime}}^{p_{2}}\right)_{i}\left(\uparrow_{p_{1}^{\prime}}^{p_{2}}\right)_{j}\right|\right\}>\frac{\varepsilon}{2} \text { for any } p_{1}^{\prime} \in U_{1} .
\end{array}
$$

(2.3.1) Due to (2.4), we can obtain that $\lambda_{p_{1}^{\prime} p_{2}^{\prime}}=m_{l}$ (similar to (2.2)) for any $p_{i}^{\prime} \in U_{i}$ once $U_{i}$ falls in $B\left(p_{i}, \frac{\varepsilon}{4}\right)$.

(2.3.2) Since $U_{2}$ is sufficiently small, we assume that $U_{2} \subset B\left(p_{2}, \frac{\varepsilon}{8}\right)$. Based on (2.3) and (2.4), a further observation is that, for any fixed $\left[p_{1} p_{2}\right]$ and any $p_{2}^{\prime} \in U_{2}$,

$$
\exists \text { ! }\left[p_{1} p_{2}^{\prime}\right] \text { s.t. }\left|\uparrow_{p_{1}}^{p_{2}} \uparrow_{p_{1}}^{p_{2}^{\prime}}\right|=\left|p_{2} p_{2}^{\prime}\right| \text {, and } \forall p_{2}^{1}, p_{2}^{2} \in U_{2},\left|\uparrow_{p_{1}}^{p_{2}^{1}} p_{p_{1}}^{p_{2}^{2}}\right|=\left|p_{2}^{1} p_{2}^{2}\right| \text {. }
$$

Due to (2.3), we need only to prove that $\left|\uparrow_{p_{1}}^{p_{2}^{1}} p_{2}^{2}\right|=\left|p_{2}^{1} p_{2}^{2}\right|$. If this is not true, by Lemma 1.4 there is another $\left(\uparrow_{p_{1}}^{p_{2}^{2}}\right)^{\prime}$ such that $\left|\uparrow_{p_{1}}^{p_{2}^{1}}\left(\uparrow_{p_{1}}^{p_{2}^{2}}\right)^{\prime}\right|=\left|p_{2}^{1} p_{2}^{2}\right|$. It then follows that $\left|\uparrow_{p_{1}}^{p_{2}^{2}}\left(\uparrow_{p_{1}}^{p_{2}^{2}}\right)^{\prime}\right| \leq\left|p_{2}^{2} p_{2}\right|+\left|p_{2} p_{2}^{1}\right|+\left|p_{2}^{1} p_{2}^{2}\right|<\frac{\varepsilon}{2}$, which contradicts (2.4).

Note that (2.5) implies that $f_{\left[p_{1} p_{2}\right]}: U_{2} \rightarrow\left(\Sigma_{p_{1}} X_{1}\right)^{\perp}$ is an isometrical embedding, and similarly, so is $f_{\left[p_{2} p_{1}\right]}: U_{1} \rightarrow\left(\Sigma_{p_{2}} X_{2}\right)^{\perp}$ for sufficiently small $U_{1}$.

(2.3.3) We still consider the fixed $\left[p_{1} p_{2}\right]$, and assume that $U_{i} \subset B\left(p_{i}, \frac{\varepsilon}{8}\right)(i=1,2)$. For any $p_{2}^{\prime} \in U_{2}$, let $\left[p_{1} p_{2}^{\prime}\right]$ be the minimal geodesic determined in (2.5). Similarly, for any $p_{1}^{\prime} \in U_{1}$,

$$
\exists !\left[p_{1}^{\prime} p_{2}^{\prime}\right] \text { s.t. }\left|\uparrow_{p_{2}^{\prime}}^{p_{1}} \uparrow_{p_{2}^{\prime}}^{p_{1}^{\prime}}\right|=\left|p_{1} p_{1}^{\prime}\right| \text {, and } \forall p_{1}^{1}, p_{1}^{2} \in U_{1},\left|\uparrow_{p_{2}^{\prime}}^{p_{1}^{1}} p_{p_{2}^{\prime}}^{2}\right|=\left|p_{1}^{1} p_{1}^{2}\right| \text {. }
$$

For the $\left[p_{1} p_{2}\right]$ and $\left[p_{1}^{\prime} p_{2}^{\prime}\right]$, we define

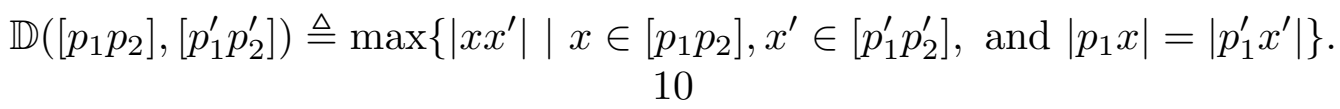


It is not hard to see that

$$
\mathbb{D}\left(\left[p_{1} p_{2}\right],\left[p_{1}^{\prime} p_{2}^{\prime}\right]\right) \leq \mathbb{D}\left(\left[p_{1} p_{2}\right],\left[p_{1} p_{2}^{\prime}\right]\right)+\mathbb{D}\left(\left[p_{1} p_{2}^{\prime}\right],\left[p_{1}^{\prime} p_{2}^{\prime}\right]\right)=\left|p_{2} p_{2}^{\prime}\right|+\left|p_{1} p_{1}^{\prime}\right| .
$$

Then, due to (2.1), there is $\nu>0$ such that if $\left|p_{2} p_{2}^{\prime}\right|+\left|p_{1} p_{1}^{\prime}\right|<\nu$, then $\left[p_{1}^{\prime} p_{2}^{\prime}\right]$ is the unique minimal geodesic between $p_{1}^{\prime}$ and $p_{2}^{\prime}$ satisfying

$$
\mathbb{D}\left(\left[p_{1} p_{2}\right],\left[p_{1}^{\prime} p_{2}^{\prime}\right]\right)<\nu \text {. }
$$

Based on (2.8), we claim that for sufficiently small $U_{1}$ and $U_{2}$, the $\left[p_{1}^{\prime} p_{2}^{k}\right]$ determined in (2.6) satisfies

$$
\left|\uparrow_{p_{1}^{\prime} \uparrow}^{p_{p_{1}^{\prime}}^{1} p_{2}^{2}}\right|=\left|p_{2}^{1} p_{2}^{2}\right|
$$

In fact, by Lemma 1.4 there exists a $\left[p_{1}^{\prime} p_{2}^{1}\right]^{\prime}$ such that $\left|\left(\uparrow_{p_{1}^{\prime}}^{p_{1}^{1}}\right)^{\prime} \uparrow_{p_{1}^{\prime}}^{p_{2}^{2}}\right|=\left|p_{2}^{1} p_{2}^{2}\right|$, so

$$
\mathbb{D}\left(\left[p_{1} p_{2}\right],\left[p_{1}^{\prime} p_{2}^{1}\right]^{\prime}\right) \leq \mathbb{D}\left(\left[p_{1} p_{2}\right],\left[p_{1}^{\prime} p_{2}^{2}\right]\right)+\mathbb{D}\left(\left[p_{1}^{\prime} p_{2}^{2}\right],\left[p_{1}^{\prime} p_{2}^{1}\right]^{\prime}\right) \leq\left|p_{1} p_{1}^{\prime}\right|+\left|p_{2} p_{2}^{2}\right|+\left|p_{2}^{1} p_{2}^{2}\right|
$$

Hence, once $U_{1}$ and $U_{2}$ are so small that $\left|p_{1} p_{1}^{\prime}\right|+\left|p_{2} p_{2}^{2}\right|+\left|p_{2}^{1} p_{2}^{2}\right|<\nu,\left[p_{1}^{\prime} p_{2}^{1}\right]^{\prime}$ has to be the $\left[p_{1}^{\prime} p_{2}^{1}\right]$ determined in (2.6). That is, the claim is true.

Now, for the $\left[p_{1} p_{2}\right]$, it suffices to show that $U_{\left[p_{1} p_{2}\right]} \triangleq \bigcup_{p_{i}^{\prime} \in U_{i}}\left[p_{1}^{\prime} p_{2}^{\prime}\right]$ is isometric to $U_{1} * U_{2}$ (where $U_{1}$ and $U_{2}$ are in the above claim and $\left[p_{1}^{\prime} p_{2}^{\prime}\right]$ is in (2.6)), i.e., for any $x_{k} \in\left[p_{1}^{k} p_{2}^{k}\right] \subset U_{\left[p_{1} p_{2}\right]}$ with $p_{i}^{k} \in U_{i}(k=1,2)$,

$$
\left|x_{1} x_{2}\right|=\left|x_{1} x_{2}\right|_{*}
$$

where $|\cdot|_{*}$ denotes the distance of $U_{1} * U_{2}$ (note that $x_{k}$ can be regarded as the point in the $\left[p_{1}^{k} p_{2}^{k}\right] \subset U_{1} * U_{2}$ with $\left|p_{1}^{k} x_{k}\right|_{*}=\left|p_{1}^{k} x_{k}\right|$ ). By (2.9) (and its proof) and Lemma 1.4, $\left[p_{1}^{2} p_{2}^{1}\right],\left[p_{1}^{2} p_{2}^{2}\right]$ and any $\left[p_{2}^{1} p_{2}^{2}\right]$ bound a convex spherical surface, denoted by $\overline{\triangle p_{1}^{2} p_{2}^{1} p_{2}^{2}}$. This implies that $\left|p_{2}^{1} x_{2}\right|=\left|p_{2}^{1} x_{2}\right|_{*}$ (similarly, $\left|p_{1}^{1} x_{2}\right|=\left|p_{1}^{1} x_{2}\right|_{*}$ ); and for any $z \in\left[p_{2}^{1} x_{2}\right] \subset \overline{\triangle p_{1}^{2} p_{2}^{1} p_{2}^{2}}$, there is $p_{2}^{3} \in\left[p_{2}^{1} p_{2}^{2}\right]$ such that $z \in\left[p_{1}^{2} p_{2}^{3}\right] \subset \overline{\triangle p_{1}^{2} p_{2}^{1} p_{2}^{2}}$. Note that

$$
\mathbb{D}\left(\left[p_{1} p_{2}\right],\left[p_{1}^{2} p_{2}^{3}\right]\right) \leq \mathbb{D}\left(\left[p_{1} p_{2}\right],\left[p_{1}^{2} p_{2}^{2}\right]\right)+\mathbb{D}\left(\left[p_{1}^{2} p_{2}^{2}\right],\left[p_{1}^{2} p_{2}^{3}\right]\right) \leq\left|p_{1} p_{1}^{2}\right|+\left|p_{2} p_{2}^{2}\right|+\left|p_{2}^{2} p_{2}^{1}\right|,
$$

so it has to hold that $\left[p_{1}^{2} p_{2}^{3}\right] \subset U_{\left[p_{1} p_{2}\right]}$ (see (2.8)). Similarly, there is a convex spherical surface $\overline{\triangle p_{1}^{1} p_{1}^{2} p_{2}^{3}}$ which contains $\left[p_{1}^{2} p_{2}^{3}\right]$, and so $\left|p_{1}^{1} z\right|=\left|p_{1}^{1} z\right|_{*}$ (note that $z$ can be regarded as the point in the $\left[p_{2}^{1} x_{2}\right] \subset U_{1} * U_{2}$ with $\left.\left|p_{2}^{1} z\right|_{*}=\left|p_{2}^{1} z\right|\right)$. Hence, by (1.1.3), $\left[p_{1}^{1} z\right]\left(\subset \overline{\triangle p_{1}^{1} p_{1}^{2} p_{2}^{3}}\right)$ and $\left[p_{2}^{1} x_{2}\right]$ determine a convex spherical surface $\overline{\triangle p_{1}^{1} p_{2}^{1} x_{2}}$ $\left(\supset\left[p_{1}^{1} z\right]\right)$ bounded by $\left[p_{2}^{1} x_{2}\right]$ and some $\left[p_{1}^{1} p_{2}^{1}\right]^{\prime}$ and $\left[p_{1}^{1} x_{2}\right]$. Similarly, for any $x \in$ $\left[p_{1}^{1} p_{2}^{1}\right]^{\prime}$, we have that

$$
\left|x_{2} x\right|=\left|x_{2} x\right|_{*},
$$

which will imply $(2.10)$ as long as we prove that $\left[p_{1}^{1} p_{2}^{1}\right]^{\prime}$ is just the $\left[p_{1}^{1} p_{2}^{1}\right]$. Note that $\left[p_{1}^{1} z\right] \subseteq \overline{\triangle p_{1}^{1} p_{1}^{2} p_{2}^{3}} \cap \overline{\triangle p_{1}^{1} p_{2}^{1} x_{2}}$ and $\left[p_{2}^{1} z\right] \subseteq \overline{\triangle p_{1}^{2} p_{2}^{1} p_{2}^{2}} \cap \overline{\triangle p_{1}^{1} p_{2}^{1} x_{2}}$, so it is not hard to see that $\mathbb{D}\left(\left[p_{1} p_{2}\right]^{\prime},\left[p_{1}^{2} p_{2}^{3}\right]\right)<\nu / 2$ for sufficiently small $U_{1}$ and $U_{2}$ (where $\nu$ is the number in $(2.8))$. And we can assume that $\mathbb{D}\left(\left[p_{1} p_{2}\right],\left[p_{1}^{2} p_{2}^{3}\right]\right)<\nu / 2$ (see (2.7-8)), so

$$
\mathbb{D}\left(\left[p_{1} p_{2}\right],\left[p_{1}^{1} p_{2}^{1}\right]^{\prime}\right) \leq \mathbb{D}\left(\left[p_{1} p_{2}\right],\left[p_{1}^{2} p_{2}^{3}\right]\right)+\mathbb{D}\left(\left[p_{1}^{2} p_{2}^{3}\right],\left[p_{1}^{1} p_{2}^{1}\right]^{\prime}\right)<\nu
$$


Then, by (2.8), $\left[p_{1}^{1} p_{2}^{1}\right]^{\prime}$ has to be the $\left[p_{1}^{1} p_{2}^{1}\right]$ determined in (2.6).

Remark 2.4. The above argument can be used to prove a weak version of 'local' join structure: for any $\left[p_{1} p_{2}\right]$ with $p_{i} \in X_{i}^{\circ}$ and $\lambda_{p_{1} p_{2}}<m$, given $\left[p_{1}^{\prime} p_{2}^{\prime}\right]$ with $p_{i}^{\prime} \in X_{i}^{\circ}$ and $\mathbb{D}\left(\left[p_{1} p_{2}\right],\left[p_{1}^{\prime} p_{2}^{\prime}\right]\right)$ sufficiently small, there is $\left[p_{i} p_{i}^{\prime}\right] \subset X_{i}$ such that $\left[p_{1} p_{2}\right] \cup\left[p_{1}^{\prime} p_{2}^{\prime}\right]$ can be embedded isometrically into $\left[p_{1} p_{1}^{\prime}\right] *\left[p_{2} p_{2}^{\prime}\right]$, i.e., for any $x \in\left[p_{1} p_{2}\right]^{\circ}$ and $x^{\prime} \in\left[p_{1}^{\prime} p_{2}^{\prime}\right]^{\circ}$,

$$
\left|x x^{\prime}\right|=\left|x x^{\prime}\right|_{*} \quad(\text { similar to }(2.10)),
$$

where $|\cdot|_{*}$ denotes the distance of $\left[p_{1} p_{1}^{\prime}\right] *\left[p_{2} p_{2}^{\prime}\right]$ (note that here $\left[p_{1} p_{1}^{\prime}\right]$ and $\left[p_{2} p_{2}^{\prime}\right]$ can be chosen arbitrarily). In what follows, we point out that, for any $\left[x x^{\prime}\right]$, the above underlying join structure implies a canonical way to locate the projection of $\left[x x^{\prime}\right]$ on $X_{i}{ }^{6}$, and the projection is a minimal geodesic $\left[p_{i} p_{i}^{\prime}\right]$. Indeed, the way to locate the projection here is the same as to do this in $\left[p_{1} p_{1}^{\prime}\right] *\left[p_{2} p_{2}^{\prime}\right]$ (see below for a step-by-step picture).
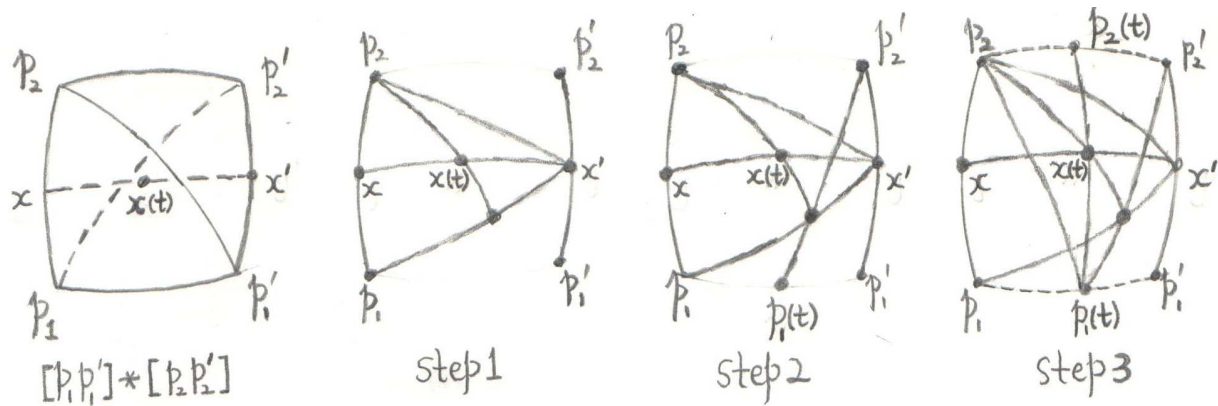

Proof of Key Lemma 1.6.

By Lemma 2.1, $\lambda_{p_{1} p_{2}}$ has a maximum $m$ for all $p_{i} \in X_{i}^{\circ}$. And by (2.3.1), $X_{i}^{m}\left(=\left\{p \in X_{i}^{\circ} \mid \exists q \in X_{j}^{\circ}(j \neq i)\right.\right.$ s.t. $\left.\left.\lambda_{p q}=m\right\}\right)$ is open in $X^{\circ}$. Hence, it suffices to show that $X_{i}^{m}$ is dense in $X_{i}^{\circ}$ and that $\lambda_{p_{1} p_{2}}=m$ for all $p_{i} \in X_{i}^{m}$. We will give a proof by induction on $n$, starting with the trivial case, $n=1$.

We first prove that for all $p_{i} \in X_{i}^{m}, \lambda_{p_{1} p_{2}}=m$. By the definition of $X_{i}^{m}$, there is a $p_{2}^{\prime} \in X_{2}^{\circ}$ and $p_{1}^{\prime} \in X_{1}^{\circ}$ such that $\lambda_{p_{1} p_{2}^{\prime}}=m=\lambda_{p_{1}^{\prime} p_{2}}$. According to (2.3.3), there are neighborhoods $U_{i} \subset X_{i}^{\circ}$ of $p_{1}$ and $p_{2}^{\prime}$ such that $U_{1} * U_{2}$ can be embedded isometrically into $X$ around any given $\left[p_{1} p_{2}^{\prime}\right]$.

We consider $\Sigma_{p_{1}} X \in \operatorname{Alex}^{n-1}(1)$, in which both $\Sigma_{p_{1}} X_{1}$ and $\left(\Sigma_{p_{1}} X_{1}\right)^{\perp}$ are convex (see (1.5)). By Remark 1.9, $\operatorname{dim}\left(\Sigma_{p_{1}} X_{1}\right)+\operatorname{dim}\left(\left(\Sigma_{p_{1}} X_{1}\right)^{\perp}\right)=\operatorname{dim}\left(\Sigma_{p_{1}} X\right)-1$. Hence, by the inductive assumption on $\Sigma_{p_{1}} X, \lambda_{\xi \zeta}$ has a maximum $m^{\prime}$ for any $\xi \in\left(\Sigma_{p_{1}} X_{1}\right)^{\circ}$ $\left(=\Sigma_{p_{1}} X_{1}\right)$ and $\zeta \in\left(\left(\Sigma_{p_{1}} X_{1}\right)^{\perp}\right)^{\circ}$, and $\lambda_{\xi \zeta}=m^{\prime}$ for any $\xi \in\left(\Sigma_{p_{1}} X_{1}\right)^{m^{\prime}}$ and $\zeta \in\left(\left(\Sigma_{p_{1}} X_{1}\right)^{\perp}\right)^{m^{\prime}}$. On the other hand, by (2.3.2), $f_{\left[p_{1} p_{2}^{\prime}\right]}: U_{2} \longrightarrow\left(\Sigma_{p_{1}} X_{1}\right)^{\perp}$ is an isometrical embedding, which implies that $\uparrow_{p_{1}}^{p_{2}^{\prime}} \in\left(\left(\Sigma_{p_{1}} X_{1}\right)^{\perp}\right)^{\circ}$ (note that $\operatorname{dim}\left(\left(\Sigma_{p_{1}} X_{1}\right)^{\perp}\right)=\operatorname{dim}\left(X_{2}\right)$ and $\left.p_{2}^{\prime} \in X_{2}^{\circ}\right)$. Then that $U_{1} * U_{2}$ can be embedded isometrically into $X$ around $\left[p_{1} p_{2}^{\prime}\right]$ implies that

$$
m^{\prime}=1
$$

Let $p_{2}^{\prime \prime}$ be an $\left(n_{2}, \delta\right)$-strained point in $X_{2}$ closed to $p_{2}$. We first show that $\lambda_{p_{1} p_{2}^{\prime \prime}}=m$. From the proof of (2.1.2), we know that $\uparrow_{p_{1}}^{p_{2}^{\prime \prime}} \in\left(\left(\Sigma_{p_{1}} X_{1}\right)^{\perp}\right)^{\circ}$ for any

\footnotetext{
${ }^{6}$ For any $x \in\left[x_{1} x_{2}\right]^{\circ}$ with $x_{i} \in X_{i}$, we say that $x_{i}$ is the projection of $x$ on $X_{i}$.
} 
$\left[p_{1} p_{2}^{\prime \prime}\right]$ (however, so far we do NOT know that $\uparrow_{p_{1}}^{p_{2}} \in\left(\left(\Sigma_{p_{1}} X_{1}\right)^{\perp}\right)^{\circ}$ for a $\left.\left[p_{1} p_{2}\right]\right)$. Note that $\lambda_{p_{1}^{\prime} p_{2}^{\prime \prime}}=m$ by (2.3.1). If $\lambda_{p_{1} p_{2}^{\prime \prime}}<m$, then by Lemma 1.4 there is some $\uparrow_{p_{1}}^{p_{1}^{\prime}}$ and $\uparrow_{p_{1}}^{p_{2}^{\prime \prime}}$ such that $\lambda_{\uparrow_{p_{1}}^{p_{1}^{\prime}} \uparrow_{p_{1}}^{p^{\prime \prime}}}>1$, which contradicts $(2.11)$.

Now, we show that $\lambda_{p_{1} p_{2}}=m$. Since $\lambda_{p_{1} p_{2}^{\prime \prime}}=m$, if $\lambda_{p_{1} p_{2}}<m$, then by Lemma 1.4 there are $\left[p_{1} p_{2}^{\prime \prime}\right]_{k}(k=1,2)$ and $\left[p_{1} p_{2}\right]$ such that $\left|\uparrow_{p_{1}}^{p_{2}}\left(\uparrow_{p_{1}}^{p_{2}^{\prime \prime}}\right)_{k}\right|=\left|p_{2} p_{2}^{\prime \prime}\right|$. Note that there is a unique minimal geodesic between $\left(\uparrow_{p_{1}^{2}}^{p_{2}^{\prime \prime}}\right)_{k} \in\left(\left(\Sigma_{p_{1}} X_{1}\right)^{\perp}\right)^{\circ}$ and given $\uparrow_{p_{1}}^{p_{1}^{\prime}} \in \Sigma_{p_{1}} X$ (see (2.11)), so by applying Lemma 1.4 on $\Sigma_{p_{1}} X$ we get that

$$
\mathbb{D}\left(\left[\uparrow_{p_{1}}^{p_{1}^{\prime}}\left(\uparrow_{p_{1}}^{p_{2}^{\prime \prime}}\right)_{1}\right],\left[\uparrow_{p_{1}}^{p_{1}^{\prime}}\left(\uparrow_{p_{1}}^{p_{2}^{\prime \prime}}\right)_{2}\right]\right)=\left|\left(\uparrow_{p_{1}}^{p_{2}^{\prime \prime}}\right)_{1}\left(\uparrow_{p_{1}}^{p_{2}^{\prime \prime}}\right)_{2}\right| \leq 2\left|p_{2} p_{2}^{\prime \prime}\right| .
$$

On the other hand, still by Lemma 1.4 , there is $\left[p_{1}^{\prime} p_{2}^{\prime \prime}\right]_{k}$ such that $\left[\uparrow_{p_{1}}^{p_{1}^{\prime}}\left(\uparrow_{p_{1}}^{p_{2}^{\prime \prime}}\right)_{k}\right]$ $\left(\subset \Sigma_{p_{1}} X\right)$ is realized by the convex spherical surface $S_{k}$ bounded by $\left[p_{1} p_{2}^{\prime \prime}\right]_{k},\left[p_{1}^{\prime} p_{2}^{\prime \prime}\right]_{k}$ and $\left[p_{1} p_{1}^{\prime}\right]$. Let $x_{k} \in\left[p_{1}^{\prime} p_{2}^{\prime \prime}\right]_{k}$ with $\left|p_{1}^{\prime} x_{1}\right|=\left|p_{1}^{\prime} x_{2}\right|$, and $\left[p_{1} x_{k}\right] \subset S_{k}$. Note that $\left|p_{1} x_{1}\right|=\left|p_{1} x_{2}\right|$, so $\left|\uparrow_{p_{1}}^{x_{1}} \uparrow_{p_{1}}^{x_{2}}\right| \geq\left|x_{1} x_{2}\right|$ by (1.1.2). This implies that

$$
\mathbb{D}\left(\left[\uparrow_{p_{1}}^{p_{1}^{\prime}}\left(\uparrow_{p_{1}}^{p_{2}^{\prime \prime}}\right)_{1}\right],\left[\uparrow_{p_{1}}^{p_{1}^{\prime}}\left(\uparrow_{p_{1}}^{p_{2}^{\prime \prime}}\right)_{2}\right]\right) \geq \mathbb{D}\left(\left[p_{1}^{\prime} p_{2}^{\prime \prime}\right]_{1},\left[p_{1}^{\prime} p_{2}^{\prime \prime}\right]_{2}\right),
$$

which contradicts $(2.12)$ (because $\left|p_{2} p_{2}^{\prime \prime}\right|$ can be sufficiently small, but $\mathbb{D}\left(\left[p_{1}^{\prime} p_{2}^{\prime \prime}\right]_{1}\right.$, $\left[p_{1}^{\prime} p_{2}^{\prime \prime}\right]_{2}$ ) has a positive lower bound (see (2.4))).

We then prove that $X_{i}^{m}$ is dense in in $X_{i}^{\circ}$. If this is not true, then there is $q_{i} \in X_{i}^{\circ}$ such that $\lambda_{q_{1} q_{2}}=m^{\prime}<m$ is a local maximum. Similar to (2.11), we can conclude that there is a unique minimal geodesic between any point in $\Sigma_{q_{i}} X_{i}$ and in $\left(\left(\Sigma_{q_{i}} X_{i}\right)^{\perp}\right)^{\circ}$. Now, consider a pair of $p_{i} \in X_{i}^{m}$. By (2.3.1), $p_{i}, q_{i}$ can be chosen to be $\left(n_{i}, \delta\right)$-stained points. Note that, when $\lambda_{p_{1} q_{2}}=m\left(\right.$ resp. $\left.\lambda_{p_{1} q_{2}}<m\right)$, by Lemma 1.4, there is some $\uparrow_{q_{1}}^{p_{1}} \in \Sigma_{q_{1}} X_{1}$ and $\uparrow_{q_{1}}^{q_{2}} \in\left(\left(\Sigma_{q_{1}} X_{1}\right)^{\perp}\right)^{\circ}$ (resp. $\uparrow_{q_{2}}^{p_{2}} \in \Sigma_{q_{2}} X_{2}$ and $\left.\uparrow_{q_{2}}^{p_{1}} \in\left(\left(\Sigma_{q_{2}} X_{2}\right)^{\perp}\right)^{\circ}\right)$ between which there are at least two minimal geodesics; a contradiction.

We will end this section with some properties of $X_{i}^{m}$, which will be used in Section 4.

Corollary 2.5. For any $p_{i} \in X_{i}^{m}$, if $\left(\Sigma_{p_{i}} X_{i}\right)^{\perp}$ has an empty boundary, then $\Sigma_{p_{i}} X=\Sigma_{p_{i}} X_{i} *\left(\Sigma_{p_{i}} X_{i}\right)^{\perp}$.

Note that Corollary 2.5 can be seen from the proof around (2.11).

Lemma 2.6. For any $p_{1} \in X_{1}^{\circ}$ and $\left[p_{2} p_{2}^{\prime}\right] \subset X_{2}^{\circ}$, there exists a finite number $m_{c}$ such that $\lambda_{p_{1} p_{2}^{\prime \prime}}=m_{c}$ for all $p_{2}^{\prime \prime} \in\left[p_{2} p_{2}^{\prime}\right]^{\circ}$, and $\lambda_{p_{1} p_{2}}, \lambda_{p_{1} p_{2}^{\prime}} \leq m_{c}$.

Proof. By Lemma 1.4 (resp. (1.1.3)), any $\left[p_{1} p_{2}\right]$ (resp. $\left[p_{1} p_{2}^{\prime \prime}\right]$ ) has to lie in a convex spherical surface bounded by $\left[p_{1} p_{2}\right],\left[p_{2} p_{2}^{\prime}\right]$ and some $\left[p_{1} p_{2}^{\prime}\right]$. Moreover, the interior parts of two such surfaces do not intersect. This together with Lemma 2.1 implies the lemma.

Lemma 2.6 has an immediate corollary.

Corollary 2.7. (2.7.1) For any $p_{i} \in X_{i}^{m}$ and $\left[p_{i} p_{i}^{\prime}\right] \subset X_{i},\left[p_{i} p_{i}^{\prime}\right] \backslash\left\{p_{i}^{\prime}\right\}$ belongs to $X_{i}^{m}$.

(2.7.2) $X_{i}^{m}$ is totally convex in $X_{i}$. 


\section{Proof of Key Lemma 1.7}

In the proof Key Lemma 1.7, we will show that the following multi-valued map

$$
f_{p_{1}}: X_{2} \rightarrow\left(\Sigma_{p_{1}} X_{1}\right)^{\perp} \quad \text { by } p_{2} \mapsto \Uparrow_{p_{1}}^{p_{2}}
$$

is onto if $\partial X_{2}=\emptyset$, where $p_{1} \in X_{1}^{\circ}$. It suffices to check that $f_{p_{1}}$ is an open map (it is clear that $f_{p_{1}}$ is a closed map). Note that at any $\left(n_{2}, \delta\right)$-strained point, we have already known that $f_{p_{1}}$ is open (see the arguments after (2.11)).

To prove the openness for $f_{p_{1}}$, we introduce the concept of cone-neighborhood isometrical multi-valued map.

Definition 3.1. Let $X, \tilde{X} \in \operatorname{Alex}(1)$ with $\operatorname{dim}(\tilde{X}) \geq \operatorname{dim}(X) \geq 1$, and let $f: X \rightarrow$ $\tilde{X}$ be a multi-valued map. We say that $f$ is a cone-neighborhood isometry if the following hold:

(3.1.1) there is an $m$ such that $\#\{f(p)\} \leq m$ for all $p \in X$, and that $X_{m} \triangleq\{p \in$ $X \mid \#\{f(p)\}=m\}$ is dense in $X$.

(3.1.2) $|\tilde{p} \tilde{q}| \geq|p q|$ for any $p, q \in X, \tilde{p} \in f(p)$ and $\tilde{q} \in f(q)$; and given any $[p q]$ and $\tilde{p} \in f(p)$, there exists a $[\tilde{p} \tilde{q}]$ with $\tilde{q} \in f(q)$ such that $[\tilde{p} \tilde{q}] \subseteq f([p q])$ and $\left.f\right|_{[p q]}:[p q] \rightarrow[\tilde{p} \tilde{q}]$ is an isometry.

Remark 3.2. For any $p \in X$ and $\tilde{p} \in f(p)$ in Definition 3.1, let $r_{\tilde{p}} \triangleq \frac{1}{4} \min \left\{\left|\tilde{p} \tilde{p}^{\prime}\right| \mid \tilde{p}^{\prime} \in\right.$ $\left.f(p), \tilde{p}^{\prime} \neq \tilde{p}\right\}$. Then it follows from (3.1.1) and (3.1.2) that:

(3.2.1) if $p \in X_{m}$, then $f: B\left(p, r_{\tilde{p}}\right) \rightarrow B\left(\tilde{p}, r_{\tilde{p}}\right)$ is an isometrical embedding;

(3.2.2) if $p \in X \backslash X_{m}$, then $|\tilde{p} \tilde{q}|=|p q|$ for any $q \in B\left(p, r_{\tilde{p}}\right)$ and $\tilde{q} \in f(q) \cap B\left(\tilde{p}, r_{\tilde{p}}\right)$.

Why do we call such $f$ a cone-neighborhood isometry? To give an explanation, we first define the $\varepsilon$-cone neighborhood $V_{[p q], \varepsilon}$ of any given $[p q] \subset X$ as follows:

$$
V_{[p q], \varepsilon} \triangleq\left\{x \in X|| x p|\leq| q p \mid \text { and there is a }[p x] \text { s.t. }\left|\uparrow_{p}^{x} \uparrow_{p}^{q}\right|<\epsilon\right\}
$$

From Definition 3.1 (and Remark 3.2), for any $\tilde{p} \in f(p)$ and $[p q] \subset B\left(p, r_{\tilde{p}}\right)$, it is easy to see that there are minimal geodesics $\left\{\left[\tilde{p} \tilde{q}_{i}\right]\right\}_{i=1}^{k}(k \leq m)$ with $\tilde{q}_{i} \in f(q) \cap B\left(\tilde{p}, r_{\tilde{p}}\right)$ such that

$$
B\left(\tilde{p}, r_{\tilde{p}}\right) \cap f([p q])=\bigcup_{i=1}^{k}\left[\tilde{p} \tilde{q}_{i}\right]
$$

We know that any triangle $\triangle \tilde{x} \tilde{p} \tilde{y}$, where $\tilde{x} \in\left[\tilde{p} \tilde{q}_{i}\right]$ and $\tilde{y} \in\left[\tilde{p} \tilde{q}_{j}\right]$, will be more and more isometric to its comparison triangle as $\tilde{x}, \tilde{y} \rightarrow \tilde{p}$ ([BGP $]$ ). Hence, it is not hard to see that there is an $N>4$ such that for each $\uparrow \uparrow_{\tilde{p}}^{\tilde{q}_{i}}$

$$
r_{\uparrow_{\tilde{p}}^{\tilde{q}_{i}}} \triangleq \frac{1}{N} \min _{j \neq i}\left\{\left|\uparrow_{\tilde{p}}^{\tilde{q}_{i} \uparrow_{\tilde{p}}^{\tilde{q}_{j}}}\right|\right\}
$$

satisfies that, for $\tilde{q}_{i, \delta} \in\left[\tilde{p} \tilde{q}_{i}\right]$ and $q_{\delta} \in[p q]$ with $\left|\tilde{p} \tilde{q}_{i, \delta}\right|=\left|p q_{\delta}\right|=\delta$ sufficiently small,

$$
V_{\left[\tilde{p} \tilde{q}_{i, \delta}\right], r_{\uparrow_{\tilde{p}} \tilde{q}_{i}}} \subset \bigcup_{\tilde{x} \in\left[\tilde{p} \tilde{q}_{i, \delta}\right] \backslash\{\tilde{p}\}} B\left(\tilde{x}, r_{\tilde{x}}\right)
$$


and

$$
V_{\left[p q_{\delta}\right], r_{\uparrow} \tilde{q}_{\tilde{p}}} \subset \bigcup_{x \in\left[p q_{\delta}\right] \backslash\{p\}, \tilde{x} \in f(x) \cap\left[\tilde{p} \tilde{q}_{i}\right]} B\left(x, r_{\tilde{x}}\right)
$$

It then follows that

$$
f\left(V_{\left[p q_{\delta}\right], r_{\uparrow}^{\tilde{q}_{i}}}\right) \subseteq V_{\left[\tilde{p} \tilde{q}_{i, \delta}\right], r_{\uparrow}^{\tilde{q}_{i}}},
$$

due to which we call the multi-valued $f$ a cone-neighborhood isometry.

By the way, we would like to point out that (3.1) implies that

$$
[p q] \backslash\{p\} \subset X_{m}
$$

for any $[p q]$ with $q \in B\left(p, r_{p}\right) \cap X_{m}$, where $r_{p}=\min \left\{r_{\tilde{p}} \mid \tilde{p} \in f(p)\right\}$.

A substantial property of a cone-neighborhood isometry is:

Proposition 3.3. Let $f: X \rightarrow \tilde{X}$ be a cone-neighborhood isometry. Then at any $p \in X$ and $\tilde{p} \in f(p), f$ induces a tangent map $\mathrm{D} f: \Sigma_{p} X \rightarrow \Sigma_{\tilde{p}} \tilde{X}$ such that $\mathrm{D} f$ is again a cone-neighborhood isometry.

Proof. By (3.2.1), if $p \in X_{m}$, then $f$ induces naturally an isometrical embedding Df $: \Sigma_{p} X \rightarrow \Sigma_{\tilde{p}} \tilde{X}$. Hence, in the rest of the proof, we need only to consider $p \in X \backslash X_{m}$.

At first, we define $\operatorname{D} f(\eta)$ for all $\eta \in\left(\Sigma_{p} X\right)^{\prime}$. Recall that $\eta \in\left(\Sigma_{p} X\right)^{\prime}$ means that there is a $[p q]$ with $q \in B\left(p, r_{\tilde{p}}\right)$ such that $\eta=\uparrow_{p}^{q}([\mathrm{BGP}])$. Since $B\left(\tilde{p}, r_{\tilde{p}}\right) \cap f([p q])=$ $\cup_{i=1}^{k}\left[\tilde{p} \tilde{q}_{i}\right](k \leq m)$ where $\tilde{q}_{i} \in f(q) \cap B\left(\tilde{p}, r_{\tilde{p}}\right)$ (see $\left.(3.1)\right)$, we define

$$
\operatorname{Df}(\eta)=\left\{\uparrow_{\tilde{p}}^{\tilde{q}_{i}}\right\}_{i=1}^{k},
$$

on which we will first give two claims before defining $\mathrm{D} f(\zeta)$ for $\zeta \notin\left(\Sigma_{p} X\right)^{\prime}$.

Claim 1: there is an $m^{\prime}$ such that $\#\{\mathrm{D} f(\eta)\} \leq m^{\prime}$ for all $\eta \in\left(\Sigma_{p} X\right)^{\prime}$; and if there is a $[p q]$ with $q \in B\left(p, r_{\tilde{p}}\right) \cap X_{m}$ such that $\eta=\uparrow_{p}^{q}$, then $\#\{\operatorname{D} f(\eta)\}=m^{\prime}$. In order to see the claim, we fix a point $q \in B\left(p, r_{\tilde{p}}\right) \cap X_{m}$, and notice that $\#\{\operatorname{Df}(\eta)\}=$ $\#\left\{f(q) \cap B\left(\tilde{p}, r_{\tilde{p}}\right)\right\}$. By (3.1.2) and the choice of $r_{\tilde{p}}$ (in Remark 3.2), for any [zq] with $z \in B\left(p, r_{\tilde{p}}\right)$ and $\tilde{z} \in f(z) \cap B\left(\tilde{p}, r_{\tilde{p}}\right)$, there exists a $\left[\tilde{z} \tilde{q}_{i}\right]$ with $\tilde{q}_{i} \in f(q) \cap B\left(\tilde{p}, r_{\tilde{p}}\right)$ such that $\left.f\right|_{[z q]}:[z q] \rightarrow\left[\tilde{z} \tilde{q}_{i}\right]$ is an isometry. It follows that $\#\left\{f(z) \cap B\left(\tilde{p}, r_{\tilde{p}}\right)\right\} \leq$ $\#\left\{f(q) \cap B\left(\tilde{p}, r_{\tilde{p}}\right)\right\}$ (otherwise, for some $\tilde{q}_{i} \in f(q) \cap B\left(\tilde{p}, r_{\tilde{p}}\right), f([z q]) \cap B\left(\tilde{q}_{i}, r_{\tilde{q}_{i}}\right)$ consists of at least two geodesics starting from $\tilde{q}_{i}$, which contradicts (3.2.1) because $\left.q \in X_{m}\right)$. Note that this also implies that $\#\left\{f(z) \cap B\left(\tilde{p}, r_{\tilde{p}}\right)\right\}=\#\left\{f(q) \cap B\left(\tilde{p}, r_{\tilde{p}}\right)\right\}$ if $z$ also belongs to $X_{m}$. Therefore, Claim 1 follows.

Claim 2: Let $q, z \in B\left(p, r_{\tilde{p}}\right)$. For any $[p q],[p z]$ and $[\tilde{p} \tilde{z}] \subset f([p z]) \cap B\left(\tilde{p}, r_{\tilde{p}}\right)$, there is a $\left[\tilde{p} \tilde{q}_{i}\right] \subset f([p q]) \cap B\left(\tilde{p}, r_{\tilde{p}}\right)$ such that $\left|\uparrow_{\tilde{p}}^{\tilde{z}_{\tilde{p}} \tilde{q}_{i}}\right|=\left|\uparrow_{p}^{z} \uparrow_{p}^{q}\right|$. Let $x_{j} \in[p q]$ and $y_{j} \in[p z]$ with $x_{j}, y_{j} \rightarrow p$ as $j \rightarrow \infty$. We select $\tilde{y}_{j} \in f\left(y_{j}\right) \cap[\tilde{p} \tilde{z}]$. Similarly, for any $\left[y_{j} x_{j}\right]$, there is a $\left[\tilde{y}_{j} \tilde{x}_{j}\right]$ with $\tilde{x}_{j} \in f\left(x_{j}\right) \cap B\left(\tilde{p}, r_{\tilde{p}}\right)$ such that $\left.f\right|_{\left[y_{j} x_{j}\right]}:\left[y_{j} x_{j}\right] \rightarrow\left[\tilde{y}_{j} \tilde{x}_{j}\right]$ is an isometry. Passing to a subsequence, we can assume that $\tilde{x}_{j}$ falls in some $\left[\tilde{p} \tilde{q}_{i}\right] \subset f([p q]) \cap B\left(\tilde{p}, r_{\tilde{p}}\right)$. It then follows that $\left|\uparrow_{\tilde{p}}^{\tilde{z} \uparrow_{\tilde{p}}^{\tilde{q}_{i}}}\right|=\left|\uparrow_{p}^{z \uparrow_{p}^{q}}\right|$ (note that $\left|\tilde{p} \tilde{x}_{j}\right|=\left|p x_{j}\right|,\left|\tilde{p} \tilde{y}_{j}\right|=\left|p y_{j}\right|$ and $\left.\left|\tilde{x}_{j} \tilde{y}_{j}\right|=\left|x_{j} y_{j}\right|\right)$.

Next, we will define $\operatorname{D} f(\zeta)$ for all $\zeta \in \Sigma_{p} X \backslash\left(\Sigma_{p} X\right)^{\prime}$. Note that we can select $\left[p z_{j}\right]$ with $z_{j} \in B\left(p, r_{\tilde{p}}\right) \cap X_{m}$ such that $\uparrow_{p}^{z_{j}} \rightarrow \zeta$ as $j \rightarrow \infty$. 
Claim 3: $B \triangleq \cup_{j=1}^{\infty} \mathrm{D} f\left(\uparrow_{p}^{z_{j}}\right)$ has at most $m^{\prime}$ limiting points in $\Sigma_{\tilde{p}} \tilde{X}$, where $m^{\prime}$ is the number in Claim 1; and the limiting points of $B$ do not depend on the choice of $\left[p z_{j}\right]$. If the claim is not true, then we can let $\tilde{\zeta}_{1}, \cdots, \tilde{\zeta}_{m^{\prime}+1}$ be the limiting points of $B$. Note that for any $0<\epsilon \ll \min _{1 \leq i \neq i^{\prime} \leq m^{\prime}+1}\left\{\left|\tilde{\zeta}_{i} \tilde{\zeta}_{i^{\prime}}\right|\right\}$, there is a $J>0$ such that

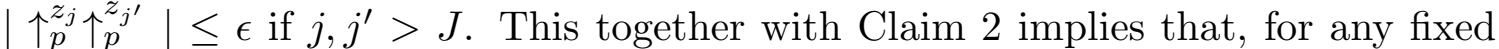
$j>J$ and $\tilde{\zeta}_{i}\left(1 \leq i \leq m^{\prime}+1\right)$, there is a $\tilde{\xi} \in \operatorname{Df}\left(\uparrow_{p}^{z_{j}}\right)$ such that $\left|\tilde{\xi}_{\zeta_{i}}\right| \leq \epsilon$. I.e., it has to hold that $\#\left\{\mathrm{D} f\left(\uparrow_{p}^{z_{j}}\right)\right\}>m^{\prime}$, which contradicts Claim 1. Hence, $B$ has at most $m^{\prime}$ limiting points; moreover, we can similarly conclude that the limiting points of $B$ do not depend on the choice of $\left[p z_{j}\right]$.

Based on Claim 3, for $\zeta \in \Sigma_{p} X \backslash\left(\Sigma_{p} X\right)^{\prime}$, we define

$$
\mathrm{D} f(\zeta)=\{\text { the limiting points of } B\}
$$

So far, we have finished the definition of a multi-valued map Df: $\Sigma_{p} X \rightarrow \Sigma_{\tilde{p}} \tilde{X}$.

At last, we need only to check that $\mathrm{D} f$ satisfies the corresponding (3.1.1-2).

"(3.1.1)": By Claim 1 and 3, it is clear that $\#\{\mathrm{D} f(\eta)\} \leq m^{\prime}$ for all $\eta \in \Sigma_{p} X$; and by the latter part of Claim $1,\left(\Sigma_{p} X\right)_{m^{\prime}} \triangleq\left\{\eta \in \Sigma_{p} X \mid \#\{\mathrm{D} f(\eta)\}=m^{\prime}\right\}$ is dense in $\Sigma_{p} X$ because $X_{m}$ is dense in $X$ and $\left(\Sigma_{p} X\right)^{\prime}$ is dense in $\Sigma_{p} X$ ([BGP]).

"(3.1.2)": For any $\eta, \zeta \in \Sigma_{p} X$, we need to prove that $|\tilde{\eta} \tilde{\zeta}| \geq|\eta \zeta|$ for any $\tilde{\eta} \in$ $\mathrm{D} f(\eta)$ and $\tilde{\zeta} \in \mathrm{D} f(\zeta)$, and that for any given $[\eta \zeta]$ and $\tilde{\eta}_{0} \in \mathrm{D} f(\eta)$ there is a $\left[\tilde{\eta}_{0} \tilde{\zeta}_{0}\right]$ with $\tilde{\zeta}_{0} \in \mathrm{D} f(\zeta)$ such that $\left.\mathrm{D} f\right|_{[\eta \zeta]}:[\eta \zeta] \rightarrow\left[\tilde{\eta}_{0} \tilde{\zeta}_{0}\right]$ is an isometry.

We first consider $\eta, \zeta \in\left(\Sigma_{p} X\right)^{\prime}$, i.e., there are $[p q],[p z] \subset B\left(p, r_{\tilde{p}}\right)$ such that $\eta=\uparrow_{p}^{q}$ and $\zeta=\uparrow_{p}^{z}$. From (3.6), there is a $[\tilde{p} \tilde{q}]$ and $[\tilde{p} \tilde{z}]$ with $\tilde{q} \in f(q) \cap B\left(\tilde{p}, r_{\tilde{p}}\right)$ and $\tilde{z} \in f(z) \cap B\left(\tilde{p}, r_{\tilde{p}}\right)$ such that $\tilde{\eta}=\uparrow_{\tilde{p}}^{\tilde{q}}$ and $\tilde{\zeta}=\uparrow_{\tilde{p}}^{\tilde{z}}$. Note that for any $\tilde{q}^{\prime} \in[\tilde{p} \tilde{q}]$ and $q^{\prime} \in f^{-1}\left(\tilde{q}^{\prime}\right)$ and any $\tilde{z}^{\prime} \in[\tilde{p} \tilde{z}]$ and $z^{\prime} \in f^{-1}\left(\tilde{z}^{\prime}\right)$, we have $\left|\tilde{q}^{\prime} \tilde{z}^{\prime}\right| \geq\left|q^{\prime} z^{\prime}\right|$ (see (3.1.2)), $\left|\tilde{p} \tilde{q}^{\prime}\right|=\left|p q^{\prime}\right|$ and $\left|\tilde{p} \tilde{z}^{\prime}\right|=\left|p z^{\prime}\right|$. It follows that $\left|\uparrow_{\tilde{p}}^{\tilde{q}} \uparrow_{\tilde{p}}^{\tilde{z}}\right| \geq\left|\uparrow_{p}^{q} \uparrow_{p}^{z}\right|$, i.e.,

$$
|\tilde{\eta} \tilde{\zeta}| \geq|\eta \zeta|
$$

Next we will find a $\left[\tilde{\eta}_{0} \tilde{\zeta}_{0}\right]$ such that $\left.\mathrm{D} f\right|_{[\eta \zeta]}:[\eta \zeta] \rightarrow\left[\tilde{\eta}_{0} \tilde{\zeta}_{0}\right]$ is an isometry. Note that $[\eta \zeta]$ can be determined by a sequence of triangles $\left\{\triangle p q_{j} z_{j}\right\}_{j=1}^{\infty}$ with $q_{j} \in[p q]$ and $z_{j} \in[p z]$ and $\left|p q_{j}\right|=\left|p z_{j}\right| \rightarrow 0$ as $j \rightarrow \infty$ (note that $\triangle p q_{j} z_{j}$ in $\left(\frac{1}{\left|p q_{j}\right|^{2}} X, p\right)$ converges to the cone $\left.C([\eta \zeta]) \subset T_{p}=C\left(\Sigma_{p} X\right)([\mathrm{BGP}])\right)$. Now, we select $\left[\tilde{p} \tilde{q}_{0}\right]$ with $\tilde{q}_{0} \in f(q) \cap B\left(\tilde{p}, r_{\tilde{p}}\right)$ such that $\tilde{\eta}_{0}=\uparrow_{\tilde{p}}^{\tilde{q}_{0}}$. By (3.1.2), for $\tilde{q}_{j} \triangleq f\left(q_{j}\right) \cap\left[\tilde{p} \tilde{q}_{0}\right]$, there is a $\left[\tilde{q}_{j} \tilde{z}_{j}\right]$ with $\tilde{z}_{j} \in f\left(z_{j}\right)$ such that $\left.f\right|_{\left[q_{j} z_{j}\right]}:\left[q_{j} z_{j}\right]\left(\subset \triangle p q_{j} z_{j}\right) \rightarrow\left[\tilde{q}_{j} \tilde{z}_{j}\right]$ is an isometry. Since $f([p z]) \cap B\left(\tilde{p}, r_{\tilde{p}}\right)$ consists of at most $m^{\prime}$ pieces of geodesics starting from $\tilde{p}$ (see Claim 1), passing to a subsequence we can assume that all $\tilde{z}_{j}$ lie in a $\left[\tilde{p} \tilde{z}_{0}\right]$ with $\tilde{z}_{0} \in f(z) \cap B\left(\tilde{p}, r_{\tilde{p}}\right)$. It is not hard to see that a subsequence of triangles $\left\{\triangle \tilde{p} \tilde{q}_{j} \tilde{z}_{j}\right\}_{j=1}^{\infty}\left(\right.$ where $\triangle \tilde{p} \tilde{q}_{j} \tilde{z}_{j}$ is formed by $\left[\tilde{p} \tilde{q}_{j}\right],\left[\tilde{p} \tilde{z}_{j}\right]$ and $\left.\left[\tilde{q}_{j} \tilde{z}_{j}\right]\right)$ determines a $\left[\tilde{\eta}_{0} \tilde{\zeta}_{0}\right]$ with $\zeta_{0}=\uparrow_{\tilde{p}}^{\tilde{z}_{0}}$ in $\Sigma_{\tilde{p}} \tilde{X}$ such that $\left.\mathrm{D} f\right|_{[\eta \zeta]}:[\eta \zeta] \rightarrow\left[\tilde{\eta}_{0} \tilde{\zeta}_{0}\right]$ is an isometry.

Now we assume that at least one of $\eta$ and $\zeta$ does not belong to $\left(\Sigma_{p} X\right)^{\prime}$, say $\eta, \zeta \notin\left(\Sigma_{p} X\right)^{\prime}$. Since we can find $\eta_{j}, \zeta_{j} \in\left(\Sigma_{p} X\right)^{\prime}$ such that $\eta_{j} \rightarrow \eta$ and $\zeta_{j} \rightarrow \zeta$ as $j \rightarrow \infty$, we can use the standard limiting argument (together with what we have proven in the above) to complete the proof.

Similar to a (single-valued) continuous map, any cone-neighborhood isometry maps a compact set to a compact set. 
Proposition 3.4. Let $f: X \rightarrow \tilde{X}$ be a cone-neighborhood isometry. If $X$ is compact, then $f(X)$ is also compact (and thus $f(X)$ is closed in $\tilde{X}$ ).

Proof. It suffices to show that any sequence $\left\{\tilde{x}_{i}\right\}_{i=1}^{\infty} \subset f(X)$ contains a subsequence which converges to a point in $f(X)$ (that $f(X)$ is closed in $\tilde{X}$ is because $\tilde{X}$ is Hausdorff and $f(X)$ is compact). Let $x_{i}=f^{-1}\left(\tilde{x}_{i}\right)$ for all $i$. Since $X$ is compact, passing to a subsequence we can assume that $x_{i} \rightarrow x$ as $i \rightarrow \infty$. Then due to (3.1.2), $\left\{\tilde{x}_{i}\right\}_{i=1}^{\infty}$ has to contain a subsequence which converges to some point of $f(x)$ (note that $\#\{f(x)\} \leq m$ ).

Based on Proposition 3.3 and 3.4, we can derive the following important property of a cone-neighborhood isometry.

Theorem 3.5. Let $X, \tilde{X} \in \operatorname{Alex}^{n}(1)$ with $n \geq 1$, and let $f: X \rightarrow \tilde{X}$ be a coneneighborhood isometry. If $X$ is compact and $\partial X=\emptyset$, then $\tilde{X}=f(X)$ (which is compact by Proposition 3.4) and $\partial \tilde{X}=\emptyset$.

Proof. We give the proof by induction on $n$. If $n=1$, then $X$ is a circle. In this case, from (3.1.2), it is easy to see that $f(X)$ is open in $\tilde{X}$ and that any point of $f(X)$ is an interior one. Moreover, $f(X)$ is compact and closed in $\tilde{X}$ by Proposition 3.4. It then follows that $\tilde{X}=f(X)$, which is compact and has an empty boundary (i.e. $\tilde{X}$ is also a circle).

Now we assume that $n>1$. Since $\mathrm{D} f: \Sigma_{x} X \rightarrow \Sigma_{\tilde{x}} \tilde{X}$ is also a cone-neighborhood isometry for any $x \in X$ and $\tilde{x} \in f(x)$ (see Proposition 3.3), by the inductive assumption (note that $\Sigma_{p} X$ is compact and has an empty boundary), we have that

$$
\mathrm{D} f\left(\Sigma_{x} X\right)=\Sigma_{\tilde{x}} \tilde{X}
$$

which is compact and has an empty boundary. It then remains to show that $\tilde{X}=$ $f(X)$. If this is not true, then, for an arbitrary $\tilde{q} \in \tilde{X} \backslash f(X)$, there is a $\tilde{p} \in f(X)$ such that (note that $f(X)$ is compact by Proposition 3.4)

$$
|\tilde{q} \tilde{p}|=\min \left\{\left|\tilde{q} \tilde{p}^{\prime}\right| \mid \tilde{p}^{\prime} \in f(X)\right\} .
$$

Select a $[\tilde{p} \tilde{q}]$. By (1.1.2) and the first variation formula, it is easy to see that there is an $\varepsilon>0$ such that

$$
V_{[\tilde{p} \tilde{q}], \varepsilon} \cap f(X)=\{\tilde{p}\},
$$

where $V_{[\tilde{p} \tilde{q}], \varepsilon}$ is the $\varepsilon$-cone neighborhood of $[\tilde{p} \tilde{q}]$. Let $p=f^{-1}(\tilde{p})$. From the definition of $\mathrm{D} f: \Sigma_{p} X \rightarrow \Sigma_{\tilde{p}} \tilde{X}$ (see Proposition 3.3 and its proof), (3.8) implies that

$$
\uparrow_{\tilde{p}}^{\tilde{q}} \notin \mathrm{D} f\left(\Sigma_{p} X\right),
$$

which contradicts (3.7) (i.e., we obtain that $f(X)=\tilde{X}$ ).

From the proof of Theorem 3.5, we get the following corollary.

Corollary 3.6. Let $X, \tilde{X} \in$ Alex $^{n}(1)$ with $n \geq 1$, and let $f: X \rightarrow \tilde{X}$ be a coneneighborhood isometry. If $p \in X^{\circ}$, then $f(p) \subset \tilde{X}^{\circ}$, and there is an $\epsilon>0$ such that $B(\tilde{p}, \epsilon) \subseteq f(B(p, \epsilon))$ for any $\tilde{p} \in f(p)$. 
Remark 3.7. Let $f: X \rightarrow \tilde{X}$ be a cone-neighborhood isometry. If $X$ is a Riemannian manifold, then $\tilde{X}=f(X)$ is a Riemannian submanifold which may have more than one component. When restricted to any component, $f^{-1}$ is a Riemannian covering map. (Hint: since $\Sigma_{x} X$ is a unit sphere for any $x \in X, \mathrm{D} f: \Sigma_{x} X \rightarrow \Sigma_{\tilde{x}} \tilde{X}$ is an isometric embedding, which together with (3.1) implies that $x \in X_{m}$.)

For general $X, \tilde{X} \in \operatorname{Alex}(1)$ ( $X$ is not a Riemannian manifold), if $\operatorname{dim}(X)=$ $\operatorname{dim}(\tilde{X})$, then $f^{-1}$ is a branch covering map (see Theorem 3.5). However, if $\operatorname{dim}(X)<\operatorname{dim}(\tilde{X})$, the above covering property of $f^{-1}$ may not hold.

Nevertheless, the following property will be used in the proof of Theorem B.

Lemma 3.8. Let $f: X \rightarrow \tilde{X}(X, \tilde{X} \in$ Alex(1)) be a cone-neighborhood isometry. If $X$ is compact and $\partial X=\emptyset$, then

$$
(f(X))^{\geq \frac{\pi}{2}}=(f(X))^{=\frac{\pi}{2}} .
$$

Proof. We give the proof by induction on $\operatorname{dim}(X)$. If $\operatorname{dim}(X)=0$, then it is obvious (because it is our convention that $X$ consists of two points with distance $\pi$, so does $f(X)$ by (3.1.2)). Now, we assume that $\operatorname{dim}(X)>0$. Let $\tilde{y}$ be an arbitrary point in $(f(X))^{\geq \frac{\pi}{2}}$, and let $\tilde{x}_{0} \in f(X)$ such that $\left|\tilde{y} \tilde{x}_{0}\right|=\min \{|\tilde{y} \tilde{x}| \mid \tilde{x} \in f(X)\}$ (see Proposition 3.4). By the first variation formula, for any $\left[\tilde{x}_{0} \tilde{y}\right]$ we have that $\uparrow_{\tilde{x}_{0}}^{\tilde{y}} \in\left(\mathrm{D} f\left(\Sigma_{x_{0}} X\right)\right)^{\geq \frac{\pi}{2}} \subset \Sigma_{\tilde{x}_{0}} \tilde{X}$ (see Proposition 3.3 for $\mathrm{D} f$ ), where $x_{0}=f^{-1}\left(\tilde{x}_{0}\right)$. By induction, we have that $\left(\mathrm{D} f\left(\Sigma_{x_{0}} X\right)\right)^{\geq \frac{\pi}{2}}=\left(\mathrm{D} f\left(\Sigma_{x_{0}} X\right)\right)=\frac{\pi}{2}$ in $\Sigma_{\tilde{x}_{0}} \tilde{X}$. Hence, $\left[\tilde{x}_{0} \tilde{y}\right]$ is perpendicular to each segment of $B\left(\tilde{x}_{0}, r_{\tilde{x}_{0}}\right) \cap f\left(\left[x_{0} z\right]\right)$ (see (3.1)) for any $\left[x_{0} z\right] \subset B\left(x_{0}, r_{\tilde{x}_{0}}\right)$. By (1.1.2) and the choice of $\tilde{x}_{0}$, it has to hold that $|\tilde{y} \tilde{z}|=\frac{\pi}{2}$ for any $\tilde{z} \in B\left(\tilde{x}_{0}, r_{\tilde{x}_{0}}\right) \cap f(X)$. It then is not hard to conclude that $|\tilde{y} \tilde{z}|=\frac{\pi}{2}$ for any $\tilde{z} \in f(X)$ (i.e. the lemma follows).

We now begin to prove Key Lemma 1.7, starting with a special case.

Lemma 3.9. Key Lemma 1.7 is true in the case where $p_{1} \in X_{1}^{m}$ (see Key Lemma 1.6 for $\left.X_{1}^{m}\right)$.

Proof. We consider the multi-valued map

$$
f_{p_{1}}: X_{2} \rightarrow\left(\Sigma_{p_{1}} X_{1}\right)^{\perp} \text { by } p_{2} \mapsto \Uparrow_{p_{1}}^{p_{2}} .
$$

By Key Lemma 1.6, Lemma 1.4 and (1.1.2), it is not hard to see that

$$
\left.f_{p_{1}}\right|_{X_{2}^{\circ}}: X_{2}^{\circ} \rightarrow\left(\Sigma_{p_{1}} X_{1}\right)^{\perp} \text { is a cone-neighborhood isometry. }
$$

Then the lemma follows from Corollary 3.6 and Theorem 3.5.

Based on Lemma 3.9, we can conclude another important property of $X_{i}^{m}$.

Lemma 3.10. For any $p_{2} \in X_{2}^{\circ}$, there exists a positive number $m_{p_{2}}(\leq m)$ such that $\lambda_{p_{1} p_{2}}=m_{p_{2}}$ for all $p_{1} \in X_{1}^{m}$ and $\lambda_{p_{1}^{\prime} p_{2}} \leq m_{p_{2}}$ for all $p_{1}^{\prime} \in X_{1}^{\circ}$. (And the similar statement holds for $p_{1} \in X_{1}^{\circ}$.)

Proof. By Lemma 3.9, for $p_{1} \in X_{1}^{m}$, any $\uparrow_{p_{1}}^{p_{2}}$ belongs to $\left(\left(\Sigma_{p_{1}} X_{1}\right)^{\perp}\right)^{\circ}$. Then (2.11) together with Lemma 1.4 implies that

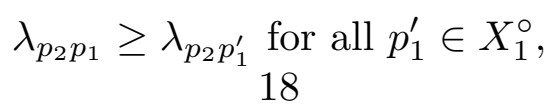


which implies the lemma.

Now, we can complete the proof of Key Lemma 1.7.

Proof of Key Lemma 1.7.

The proof is almost identical to that of Lemma 3.9 except that we should use Lemma 3.10 instead of Key Lemma 1.6 to conclude that $\left.f_{p_{1}}\right|_{X_{2}^{\circ}}: X_{2}^{\circ} \rightarrow\left(\Sigma_{p_{1}} X_{1}\right)^{\perp}$ is a cone-neighborhood isometry.

\section{Proof of Theorem A}

To prove Theorem A, by Lemma 1.2 and Theorem 1.8 it remains to prove (A2), where $X_{i}$ is compact and has an empty boundary. As outlined at the end of Introduction, for any $p_{i} \in X_{i}^{m}$ (see Key Lemma 1.6), we shall construct a finite group $\Gamma(|\Gamma|=m)$ acting isometrically on $\left(\Sigma_{p_{i}} X_{i}\right)^{\perp}$ such that $\left(\Sigma_{p_{i}} X_{i}\right)^{\perp} / \Gamma \stackrel{\text { isom }}{\cong} X_{j}$ $(i \neq j \in\{1,2\})$; and based on Key Lemma 1.7 we can define a natural map, $\left(\left(\Sigma_{p_{1}} X_{1}\right)^{\perp} *\left(\Sigma_{p_{2}} X_{2}\right)^{\perp}\right) / \Gamma \rightarrow X$, and check that the map is an isometry.

According to Key Lemma 1.7 and its proof, for any $p_{i} \in X_{i}$, we have that

$$
f_{p_{i}}: X_{j} \rightarrow\left(\Sigma_{p_{i}} X_{i}\right)^{\perp} \text { by } p_{j} \mapsto \Uparrow_{p_{i}}^{p_{j}}
$$

is a cone-neighborhood isometry $(i \neq j)$, and that $\Uparrow_{p_{i}}^{X_{j}}=\left(\Sigma_{p_{i}} X_{i}\right)^{\perp}$ which is compact and has an empty boundary. It follows that

$$
\text { For any } \zeta \in\left(\Sigma_{p_{i}} X_{i}\right)^{\perp} \text {, there is a }\left[p_{i} p_{j}\right] \text { with } p_{j} \in X_{j} \text { such that } \zeta=\uparrow_{p_{i}}^{p_{j}} \text {. }
$$

Using the above consequences of Key Lemma 1.7, we can explore a local join structure on $X$.

Lemma 4.1. (4.1.1) Any $x \in X$ belongs to some $\left[p_{1} p_{2}\right]$ with $p_{i} \in X_{i}$.

(4.1.2) For any $p_{i} \in X_{i}^{m}, \Sigma_{p_{i}} X=\Sigma_{p_{i}} X_{i} *\left(\Sigma_{p_{i}} X_{i}\right)^{\perp}$.

Proof. (4.1.1) Since $X_{1}$ is compact, there is a $p_{1} \in X_{1}$ such that $\left|x p_{1}\right|=\min \left\{\left|x p_{1}^{\prime}\right| \mid p_{1}^{\prime} \in\right.$ $\left.X_{1}\right\}$. By Lemma 1.3, we have that $\left|x p_{1}\right| \leq \frac{\pi}{2}$; and by the first variation formula, for any $\left[p_{1} x\right]$, we know that $\uparrow_{p_{1}}^{x}$ belongs to $\left(\Sigma_{p_{1}} X_{1}\right) \geq \frac{\pi}{2}$ in $\Sigma_{p_{1}} X$. Again by Lemma $1.3, \uparrow_{p_{1}}^{x} \in\left(\Sigma_{p_{1}} X_{1}\right)^{\perp}\left(=\left(\Sigma_{p_{1}} X_{1}\right)^{=\frac{\pi}{2}}\right)$, so by $(4.2)$ there exists a $\left[p_{1} p_{2}\right]$ with $p_{2} \in X_{2}$ such that $\uparrow_{p_{1}}^{x}=\uparrow_{p_{1}}^{p_{2}}$. This together with " $\left|x p_{1}\right| \leq \frac{\pi}{2}$ " implies that $\left[p_{1} x\right](\ni x)$ lies in $\left[p_{1} p_{2}\right]$.

(4.1.2) Since $\left(\Sigma_{p_{i}} X_{i}\right)^{\perp}$ has an empty boundary and $p_{i} \in X_{i}^{m}$, we conclude that $\Sigma_{p_{i}} X=\Sigma_{p_{i}} X_{i} *\left(\Sigma_{p_{i}} X_{i}\right)^{\perp}$ by Corollary 2.5 .

In view of the above, the join structure on $X$ should be independent of $p_{i} \in X_{i}^{m}$, which will be justified in Lemma 4.2.

Let $p_{1}, p_{1}^{\prime} \in X_{1}^{m}$, and $\left[p_{1} p_{1}^{\prime}\right] \subset X_{1}$. Since $\Sigma_{p_{1}} X=\Sigma_{p_{1}} X_{1} *\left(\Sigma_{p_{1}} X_{1}\right)^{\perp}$ (resp. $\left.\Sigma_{p_{1}^{\prime}} X=\Sigma_{p_{1}^{\prime}} X_{1} *\left(\Sigma_{p_{1}^{\prime}} X_{1}\right)^{\perp}\right)$ by $(4.1 .2)$, for any $p_{2} \in X_{2}$ and given $\left[p_{1} p_{2}\right]$ (resp. $\left.\left[p_{1}^{\prime} p_{2}\right]\right)$, by Lemma 1.4 there is a unique $\left[p_{1}^{\prime} p_{2}\right]$ (resp. $\left.\left[p_{1} p_{2}\right]\right)$ such that $\left[p_{1} p_{2}\right],\left[p_{1}^{\prime} p_{2}\right]$ and $\left[p_{1} p_{1}^{\prime}\right]$ bound a convex spherical surface. Hence, due to (4.2), we can define a 1-1 map

$$
h_{\left[p_{1} p_{1}^{\prime}\right]}:\left(\Sigma_{p_{1}} X_{1}\right)^{\perp} \rightarrow\left(\Sigma_{p_{1}^{\prime}} X_{1}\right)^{\perp} \text { by } \uparrow_{p_{1}}^{p_{2}} \mapsto \uparrow_{p_{1}^{\prime}}^{p_{2}} .
$$


Lemma 4.2. The map $h_{\left[p_{1} p_{1}^{\prime}\right]}:\left(\Sigma_{p_{1}} X_{1}\right)^{\perp} \rightarrow\left(\Sigma_{p_{1}^{\prime}} X_{1}\right)^{\perp}$ is an isometry.

Proof. For convenience, we let $h$ denote $h_{\left[p_{1} p_{1}^{\prime}\right]}$ in this proof. We need to show that

$$
\left|h\left(\uparrow p_{p_{1}}\right) h\left(\uparrow \uparrow_{p_{1}}^{p_{2}^{\prime}}\right)\right|=\left|\uparrow_{p_{1}}^{p_{2}} p_{p_{1}}^{p_{2}^{\prime}}\right|
$$

for any $\uparrow_{p_{1}}^{p_{2}}, \uparrow_{p_{1}}^{p_{2}^{\prime}} \in\left(\Sigma_{p_{1}} X_{1}\right)^{\perp}$. Let $\left.\tilde{\gamma}(t)\right|_{t \in[0,1]}$ be a minimal geodesic in $\left(\Sigma_{p_{1}} X_{1}\right)^{\perp}$ with $\tilde{\gamma}(0)=\uparrow_{p_{1}}^{p_{2}}$ and $\tilde{\gamma}(1)=\uparrow_{p_{1}}^{p_{2}^{\prime}}$. By (4.1) and (4.2), we can consider $\gamma(t) \triangleq f_{p_{1}}^{-1}(\tilde{\gamma}(t))$ for all $t \in[0,1]$ (note that $\gamma(0)=p_{2}$ and $\gamma(1)=p_{2}^{\prime}$ ).

Claim 1: $\left.\gamma(t)\right|_{t \in[0,1]}$ is a piecewise geodesic in $X_{2}$ with length equal to that of $\left.\tilde{\gamma}(t)\right|_{t \in[0,1]}, \mid \uparrow_{p_{1}}^{p_{2} \uparrow p_{1}^{\prime}} p_{2}^{\prime}$. This is due to that $f_{p_{1}}: X_{2} \rightarrow\left(\Sigma_{p_{1}} X_{1}\right)^{\perp}$ is a cone-neighborhood isometry and that $f_{p_{1}}$ is surjective (see Remark 3.2 and (4.1)).

Claim 2: $h$ is a continuous map. We need to show that if $\uparrow_{p_{1}}^{p_{2}^{\prime}} \rightarrow \uparrow \uparrow_{p_{1}}^{p_{2}}\left(\right.$ in $\left.\left(\Sigma_{p_{1}} X_{1}\right)^{\perp}\right)$, then $h\left(\uparrow \uparrow_{p_{1}}^{\prime}\right) \rightarrow h\left(\uparrow_{p_{1}}^{p_{2}}\right)$. Let $\uparrow_{p_{1}}^{p_{2}}, \uparrow_{p_{1}}^{p_{2}^{\prime}}, h\left(\uparrow_{p_{1}}^{p_{2}}\right)$ and $h\left(\uparrow_{p_{1}}^{p_{2}^{\prime}}\right)$ be the directions of $\left[p_{1} p_{2}\right]$, $\left[p_{1} p_{2}^{\prime}\right],\left[p_{1}^{\prime} p_{2}\right]$ and $\left[p_{1}^{\prime} p_{2}^{\prime}\right]$ respectively (see (4.2)). Note that $\Sigma_{p_{1}} X=\Sigma_{p_{1}} X_{1} *$ $\left(\Sigma_{p_{1}} X_{1}\right)^{\perp}$, so the (unique) $\left[\uparrow_{p_{1}}^{p_{2}^{\prime}} p_{p_{1}}^{p_{1}^{\prime}}\right]$ converges to the $\left[\uparrow p_{p_{1}} \uparrow_{p_{1}}^{p_{1}^{\prime}}\right]$ as $\uparrow_{p_{1}}^{p_{2}^{\prime}} \rightarrow \uparrow p_{p_{1}}^{p_{2}}$. This implies that the convex spherical surface $\overline{\triangle p_{2}^{\prime} p_{1} p_{1}^{\prime}}$ (which realizes $\left[\uparrow p_{1}^{p_{2}^{\prime}} \uparrow_{p_{1}}^{p_{1}^{\prime}}\right]$ ) bounded by $\left[p_{1} p_{2}^{\prime}\right],\left[p_{1}^{\prime} p_{2}^{\prime}\right]$ and $\left[p_{1} p_{1}^{\prime}\right]$ converges to $\overline{\triangle p_{2} p_{1} p_{1}^{\prime}}$ (which realizes $\left[\uparrow p_{p_{1}} \uparrow_{p_{1}}^{p_{1}^{\prime}}\right]$ ) bounded by $\left[p_{1} p_{2}\right],\left[p_{1}^{\prime} p_{2}\right]$ and $\left[p_{1} p_{1}^{\prime}\right]$, so $h\left(\uparrow_{p_{1}}^{p_{2}^{\prime}}\right)$ converges to $h\left(\uparrow p_{p_{1}}^{p_{2}}\right)$.

By Claim 2, $\left.\left.h(\tilde{\gamma}(t))\right|_{t \in[0,1]} \subset f_{p_{1}^{\prime}}(\gamma(t))\right|_{t \in[0,1]}$ is a continuous curve in $\left(\Sigma_{p_{1}^{\prime}} X_{1}\right)^{\perp}$, while $\left.\gamma(t)\right|_{t \in[0,1]}$ is a piecewise geodesic in $X_{2}$ by Claim 1. Note that $f_{p_{1}^{\prime}}: X_{2} \rightarrow$ $\left(\Sigma_{p_{1}^{\prime}} X_{1}\right)^{\perp}$ (defined by $p_{2}^{\prime \prime} \mapsto \Uparrow_{p_{1}^{\prime}}^{p_{2}^{\prime \prime}}$ ) is also a cone-neighborhood isometry. Hence, $\left.h(\tilde{\gamma}(t))\right|_{t \in[0,1]}$ is also a piecewise geodesic with length equal to that of $\left.\gamma(t)\right|_{t \in[0,1]}$. It then follows that

$$
\left|h\left(\uparrow p_{p_{1}}\right) h\left(\uparrow \uparrow_{p_{1}}^{p_{2}^{\prime}}\right)\right| \leq\left|\uparrow_{p_{1}}^{p_{2}} \uparrow_{p_{1}}^{p_{2}^{\prime}}\right| .
$$

In turn, we can similarly get that

$$
\left|h\left(\uparrow_{p_{1}}^{p_{2}}\right) h\left(\uparrow p_{p_{1}}^{p_{2}^{\prime}}\right)\right| \geq\left|h^{-1}\left(h\left(\uparrow_{p_{1}}^{p_{2}}\right)\right) h^{-1}\left(h\left(\uparrow \uparrow_{p_{1}}^{p_{2}^{\prime}}\right)\right)\right|=\left|\uparrow_{p_{1}}^{p_{2}} \uparrow_{p_{1}}^{p_{2}^{\prime}}\right|
$$

Hence, (4.3) follows.

Remark 4.3. From the proof of Lemma 4.2, it is not hard to see that there are neighborhoods $U_{p_{2}}, U_{\uparrow_{p_{1}}^{p_{2}}}\left(\subset\left(\Sigma_{p_{1}} X_{1}\right)^{\perp}\right)$ and $U_{\uparrow_{p_{1}^{\prime}}^{p_{2}}}\left(\subset\left(\Sigma_{p_{1}^{\prime}} X_{1}\right)^{\perp}\right)$ of $p_{2}, \uparrow_{p_{1}}^{p_{2}}$ and $\uparrow_{p_{1}^{\prime}}^{p_{2}}$ respectively such that the following diagram commutes

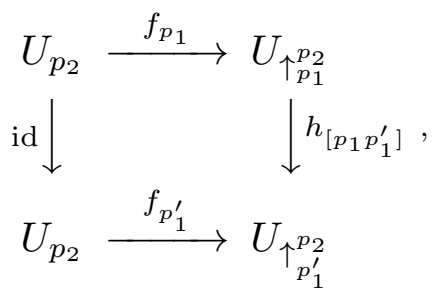

in which each map is an isometry.

Based on Lemma 4.2, we will prove the following property that is crucial to our construction of $\Gamma$. 
Lemma 4.4. For $p_{i} \in X_{i}^{m}$, any two minimal geodesics $\left[p_{1} p_{2}\right]_{1}$ and $\left[p_{1} p_{2}\right]_{2}$ determine an isometry, $\hat{\gamma}:\left(\Sigma_{p_{1}} X_{1}\right)^{\perp} \rightarrow\left(\Sigma_{p_{1}} X_{1}\right)^{\perp}$, such that $\hat{\gamma}\left(\left(\uparrow \uparrow_{p_{1}}\right)_{1}\right)=\left(\uparrow_{p_{1}}\right)_{2}$ and $\hat{\gamma}\left(\Uparrow p_{1}^{p_{2}^{\prime}}\right)=\Uparrow_{p_{1}}^{p_{2}^{\prime}}$ for $p_{2}^{\prime} \in X_{2}$.

Before giving a proof, we need some preparations.

Lemma 4.5. For $p_{1}, p_{1}^{\prime} \in X_{1}, p_{2} \in X_{2}$ and given $\left[p_{1} p_{2}\right]$ and $\left[p_{1} p_{1}^{\prime}\right]$, any $\left[\uparrow_{p_{1}}^{p_{2}} \uparrow_{p_{1}}^{p_{1}^{\prime}}\right]$ in $\Sigma_{p_{1}} X$ is realized by a convex spherical surface bounded by a triangle $\triangle p_{2} p_{1} p_{1}^{\prime}$ containing $\left[p_{1} p_{2}\right]$ and $\left[p_{1} p_{1}^{\prime}\right]$. (And the similar statement holds for given $\left[p_{2} p_{1}\right]$ and $\left.\left[p_{2} p_{2}^{\prime}\right] \subset X_{2} \cdot\right)$

Proof. Select an arbitrary $\left[\uparrow p_{2} \uparrow p_{p_{1}}^{\prime}\right]$, and let $\eta \in\left[\uparrow p_{2} \uparrow p_{p_{1}}^{p_{1}^{\prime}}\right]^{\circ}$. We first show that the lemma is true if there is a $\left[p_{1} q\right]$ such that $\uparrow_{p_{1}}^{q}=\eta$.

Claim: $\left[p_{1} q\right]$ lies in a convex spherical surface bounded by a triangle $\triangle p_{1} \bar{p}_{1} \bar{p}_{2}$ with $\bar{p}_{i} \in X_{i}$. By (4.1.1), there is a $\left[\bar{p}_{1} \bar{p}_{2}\right]$ with $\bar{p}_{i} \in X_{i}$ such that $q \in\left[\bar{p}_{1} \bar{p}_{2}\right]$. By Lemma 1.4, there is a convex spherical surface containing $p_{1}$ and $\left[\bar{p}_{1} \bar{p}_{2}\right]$. Then by (1.1.3), $\left[p_{1} q\right]$ and $\left[\bar{p}_{1} \bar{p}_{2}\right]$ determine a triangle $\triangle p_{1} \bar{p}_{1} \bar{p}_{2}$ bounding a convex spherical surface $D$ (containing $\left[p_{1} q\right]$ and $\left.\left[\bar{p}_{1} \bar{p}_{2}\right]\right)$.

We assume that the $\triangle p_{1} \bar{p}_{1} \bar{p}_{2}$ in the claim is formed by $\left[p_{1} \bar{p}_{2}\right],\left[p_{1} \bar{p}_{1}\right]$ and $\left[\bar{p}_{1} \bar{p}_{2}\right]$. It then follows from the claim that $\uparrow_{p_{1}}^{q} \in\left[\uparrow \uparrow_{p_{1}}^{p_{2}} \uparrow_{p_{1}}^{p_{1}^{\prime}}\right] \cap\left[\uparrow{ }_{p_{1}}^{\bar{p}_{2} \uparrow \bar{p}_{1}} \bar{p}_{1}\right]$, where $\left[\uparrow \bar{p}_{p_{1}} \uparrow \bar{p}_{p_{1}}\right]\left(\subset \Sigma_{p_{1}} X\right)$ is realized by $D$. Note that $\left|\uparrow_{p_{1}}^{p_{2}} \uparrow_{1}^{p_{1}^{\prime}}\right|=\left|\uparrow_{p_{1}}^{\prime} \uparrow p_{p_{1}}\right|=\left|\uparrow_{p_{1}}^{\bar{p}_{2} \uparrow p_{p_{1}}}\right|=\left|\uparrow_{p_{1}} \uparrow_{p_{1}}\right|=\frac{\pi}{2}$ in $\Sigma_{p_{1}} X$, so it has to hold that $\left[\uparrow \bar{p}_{2} \uparrow \bar{p}_{1}\right]=\left[\uparrow_{p_{1}}^{p_{2} \uparrow p_{1}^{\prime}} p_{1}^{\prime}\right]$, which implies that $\left[p_{1} p_{2}\right]=\left[p_{1} \bar{p}_{2}\right]$ and $\left[p_{1} \bar{p}_{1}\right] \supseteq\left[p_{1} p_{1}^{\prime}\right]$ or $\left[p_{1} \bar{p}_{1}\right] \subset\left[p_{1} p_{1}^{\prime}\right]$. If $\left[p_{1} \bar{p}_{1}\right] \supseteq\left[p_{1} p_{1}^{\prime}\right]$, then the proof is done. If $\left[p_{1} \bar{p}_{1}\right] \subset\left[p_{1} p_{1}^{\prime}\right]$, then, still by Lemma 1.4 , there is a $\left[p_{2} p_{1}^{\prime}\right]$ which together with $\left[p_{2} \bar{p}_{1}\right]\left(=\left[\bar{p}_{2} \bar{p}_{1}\right]\right)$ and $\left[\bar{p}_{1} p_{1}^{\prime}\right]$ bounds a convex spherical surface $D^{\prime}$. It is not hard to see that $D \cup D^{\prime}$ is a convex spherical surface (bounded by $\left[p_{1} p_{2}\right],\left[p_{1} p_{1}^{\prime}\right]$ and $\left[p_{1}^{\prime} p_{2}\right]$ ) which realizes $\left[\uparrow p_{2} \uparrow p_{1}^{p_{1}^{\prime}} p_{1}\right]$.

Next, it suffices to show that there must be a $\left[p_{1} q\right]$ such that $\uparrow_{p_{1}}^{q}=\eta$ for any $\eta \in\left[\uparrow_{p_{1}}^{p_{2}} p_{p_{1}}^{p_{1}^{\prime}}\right]^{\circ}$. If there is no $\left[p_{1} q\right]$ such that $\uparrow_{p_{1}}^{q}=\eta$, we can select $\left\{\left[p_{1} q_{j}\right]\right\}_{j=1}^{\infty}$ with $\left|p_{1} q_{j}\right| \rightarrow 0$ as $j \rightarrow \infty$ such that $\uparrow_{p_{1}}^{q_{j}} \rightarrow \eta([\mathrm{BGP}])$. By the claim above, $\left[p_{1} q_{j}\right]$ lies in a convex spherical surface $D_{j}$ bounded by a triangle $\triangle p_{1} p_{1}^{j} p_{2}^{j}$ with $p_{i}^{j} \in X_{i}$. Assume that the $\triangle p_{1} p_{1}^{j} p_{2}^{j}$ is formed by $\left[p_{1} p_{2}^{j}\right],\left[p_{1} p_{1}^{j}\right]$ and $\left[p_{1}^{j} p_{2}^{j}\right]$. Note that $D_{j}$ realizes a $\left[\uparrow_{p_{1}}^{p_{2}^{j}} \uparrow_{p_{1}}^{p_{1}^{j}}\right]$ in $\Sigma_{p_{1}} X$ with $\uparrow_{p_{1}}^{q_{j}} \in\left[\uparrow \uparrow_{p_{1}}^{p_{2}^{j}} \uparrow_{p_{1}}^{p_{1}^{j}}\right]$, so it follows from $\uparrow_{p_{1}}^{q_{j}} \rightarrow \eta$ that

$$
\left[\uparrow p_{2}^{p_{2}^{j}} p_{p_{1}}^{j}\right] \rightarrow\left[\uparrow p_{2} \uparrow p_{p_{1}}^{p_{1}^{\prime}}\right]
$$

(similar to " $\left[\uparrow \uparrow_{p_{1}}^{\bar{p}_{2} \uparrow \bar{p}_{1}} \bar{p}_{1}\right]=\left[\uparrow \uparrow_{p_{1}} \uparrow p_{1}^{p_{1}^{\prime}}\right]$ " in the above paragraph), which implies that $\left[p_{1} p_{2}^{j}\right] \rightarrow$ $\left[p_{1} p_{2}\right]$. Now we consider the cone-neighborhood isometry $f_{p_{2}^{j}}: X_{1} \rightarrow\left(\Sigma_{p_{2}^{j}} X_{2}\right)^{\perp}$ (see (4.1)). Note that $D_{j}$ realizes a $\left[\uparrow_{p_{2}^{j} \uparrow}^{p_{1}} p_{2}^{p_{1}^{j}}\right]$ which belongs to $f_{p_{2}^{j}}\left(\left[p_{1} p_{1}^{j}\right]\right)$ in $\left(\Sigma_{p_{2}^{j}} X_{2}\right)^{\perp}$. By Claim 2 in the proof of Proposition 3.3, there is a convex spherical surface $\bar{D}_{j}$ bounded by a triangle $\triangle p_{2}^{j} p_{1} p_{1}^{\prime}$ containing the $\left[p_{1} p_{2}^{j}\right]$ and $\left[p_{1} p_{1}^{\prime}\right]$ such that $\bar{D}_{j}$ realizes a $\left[\uparrow \uparrow_{p_{2}^{j}}^{p_{1} \uparrow} p_{p_{2}^{j}}^{\prime}\right]$ (belonging to $f_{p_{2}^{j}}\left(\left[p_{1} p_{1}^{\prime}\right]\right)$ in $\left.\left(\Sigma_{p_{2}^{j}} X_{2}\right)^{\perp}\right)$ with

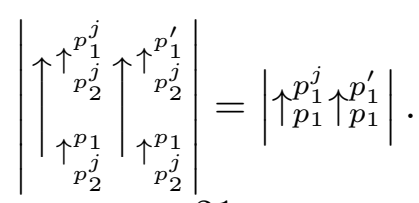


This together with " $\uparrow p_{1}^{p_{1}^{j}} \rightarrow \uparrow p_{1}^{p_{1}^{\prime}}$ " (see (4.4)) implies that the $\left[\uparrow p_{1}^{p_{2}^{j}} p_{p_{1}}^{p_{1}^{\prime}}\right]$ in $\Sigma_{p_{1}} X$ realized by $\bar{D}_{j}$ is sufficiently close to $\left[\uparrow_{2}^{p_{2}^{j}} p_{p_{1}}^{p_{1}^{j}}\right]$. It then follows from (4.4) that $\left[\uparrow_{p_{1}}^{p_{2}^{j}} \uparrow_{p_{1}}^{p_{1}^{\prime}}\right] \rightarrow$ $\left[\uparrow p_{2} \uparrow p_{1}^{p_{1}^{\prime}}\right]$, so $\bar{D}_{j}$ converges to a convex spherical surface $D$ bounded by a triangle $\triangle p_{2} p_{1} p_{1}^{\prime}$ containing the $\left[p_{1} p_{2}\right]$ and $\left[p_{1} p_{1}^{\prime}\right]$ such that the $\left[\uparrow_{p_{1}} p_{p_{1}} p_{1}^{\prime}\right]$ is realized by $D$. This contradicts the assumption that there is no $\left[p_{1} q\right]$ such that $\uparrow_{p_{1}}^{q}=\eta$.

Based on Lemma 4.5, we have a further observation.

Lemma 4.6. Let $p_{i} \in X_{i}^{m}(i=1,2)$. For any $p_{1}^{\prime} \in X_{1}, m_{p_{1}^{\prime}}$ (see Lemma 3.10 for $\left.m_{p_{1}^{\prime}}\right)$ divides $m$; and given $\left[p_{1}^{\prime} p_{2}\right]$ and $\left[p_{1}^{\prime} p_{1}\right]$, there are $\frac{m}{m_{p_{1}^{\prime}}}$ many minimal geodesics in $\Sigma_{p_{1}^{\prime}} X$ from $\uparrow_{p_{1}^{\prime}}^{p_{2}}$ to $\uparrow_{p_{1}^{\prime}}^{p_{1}}$.

Proof. At first, note that $\lambda_{p_{2} p_{1}^{\prime}}=m_{p_{1}^{\prime}}$ by Lemma 3.10. Next, note that (1.5) and Remark 1.9 enable us to apply Key Lemma 1.6 and Lemma 3.10 on $\Sigma_{p_{1}^{\prime}} X$ to conclude that there is an open and dense subset $A$ in $\left(\Sigma_{p_{1}^{\prime}} X_{1}\right)^{\perp}$ such that for any fixed $\xi \in \Sigma_{p_{1}^{\prime}} X_{1}$ there is an $m_{\xi}$ such that

$$
\lambda_{\xi \zeta}=m_{\xi} \forall \zeta \in A \text { and } \lambda_{\xi \zeta^{\prime}} \leq m_{\xi} \forall \zeta^{\prime} \in\left(\Sigma_{p_{1}^{\prime}} X_{1}\right)^{\perp}
$$

At last, note that, for any fixed $\left[p_{1} p_{2}\right]$, there is a unique $\left[p_{1}^{\prime} p_{2}\right]$ which together with $\left[p_{1} p_{2}\right]$ and $\left[p_{1} p_{1}^{\prime}\right]$ bounds a convex spherical surface (by Lemma 1.4 and (4.1.2)). Then by Lemma 4.5 , it suffices to show that, for any fixed $\left[p_{1}^{\prime} p_{2}\right]$, there are $m_{\uparrow} p_{1}$ pieces of convex spherical surfaces bounded by triangles containing $\left[p_{1}^{\prime} p_{1}\right]$ and $\left[p_{1}^{\prime} p_{2}\right]$ (which implies that $m=m_{p_{1}^{\prime}} \cdot m_{\uparrow_{p_{1}^{\prime}}^{p_{1}}}$ ).

Note that we can find $\zeta_{j} \in A$ such that $\zeta_{j} \rightarrow \uparrow_{p_{1}^{\prime}}^{p_{2}}$ as $j \rightarrow \infty$, and select $\left[p_{1}^{\prime} p_{2}^{j}\right]$ with $p_{2}^{j} \in X_{2}$ such that $\uparrow_{p_{1}^{\prime}}^{p_{2}^{j}}=\zeta_{j}$ (see $(4.2)$ ). By Lemma 4.5 , there are $\left[p_{1} p_{2}^{j}\right]_{k}$ with $1 \leq k \leq m_{\uparrow_{p_{1}^{\prime}}^{p_{1}}}$ such that the minimal geodesics between $\uparrow_{p_{1}^{\prime}}^{p_{1}}$ and $\zeta_{j}$ are realized by convex spherical surfaces $D_{k}^{j}$ bounded by $\left[p_{1} p_{1}^{\prime}\right],\left[p_{1}^{\prime} p_{2}^{j}\right]$ and $\left[p_{1} p_{2}^{j}\right]_{k}$. Since $p_{2} \in X_{2}^{m}$, we can assume that $p_{2}^{j} \in X_{2}^{m}\left(X_{2}^{m}\right.$ is open). Then by (2.4) (and Lemma 1.4), each $\left[p_{1} p_{2}^{j}\right]_{k}$ converges to some $\left[p_{1} p_{2}\right]_{k}$ as $j \rightarrow \infty$ with $\left[p_{1} p_{2}\right]_{k_{1}} \neq\left[p_{1} p_{2}\right]_{k_{2}}$ for $1 \leq k_{1} \neq k_{2} \leq m_{\uparrow_{p_{1}^{\prime}}^{p_{1}}}$. This implies that each $D_{k}^{j}$ converges to a convex spherical surface bounded by $\left[p_{1}^{\prime} p_{1}\right],\left[p_{1}^{\prime} p_{2}\right]$ and $\left[p_{1} p_{2}\right]_{k}$, so the proof is done.

We are ready to give a proof of Lemma 4.4.

Proof of Lemma 4.4.

Let $\left.\tilde{\gamma}(t)\right|_{t \in[0,1]}$ be a minimal geodesic in $\left(\Sigma_{p_{2}} X_{2}\right)^{\perp}$ with $\tilde{\gamma}(0)=\left(\uparrow_{p_{2}}\right)_{1}$ and $\tilde{\gamma}(1)=$ $\left(\uparrow_{p_{2}}\right)_{2}$; and let $\left.\gamma(t)\right|_{[0,1]} \triangleq f_{p_{2}}^{-1}\left(\left.\tilde{\gamma}(t)\right|_{[0,1]}\right)$ (note that $\gamma(0)=\gamma(1)=p_{1}$ ), which is a piecewise geodesic in $X_{1}$ with length equal to $\left|\left(\uparrow_{p_{2}}^{p_{1}}\right)_{1}\left(\uparrow_{p_{2}}^{p_{1}}\right)_{2}\right|$ (see Claim 1 in the proof of Lemma 4.2).

Case 1: $\left.\gamma(t)\right|_{[0,1]} \subset X_{1}^{m}$. Since $\left.\gamma(t)\right|_{[0,1]}$ is a piecewise geodesic, we select $0=t_{0}<$ $t_{1}<\cdots<t_{l}=1$ such that $\left.\gamma(t)\right|_{\left[t_{k}, t_{k+1}\right]}$ is a minimal geodesic for $k=0, \cdots, l-1$.

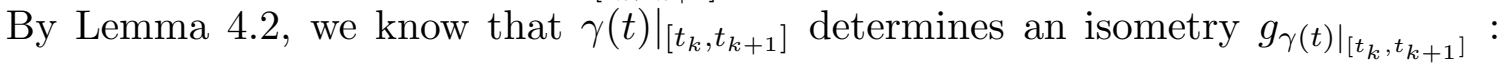
$\left(\Sigma_{\gamma\left(t_{k}\right)} X_{1}\right)^{\perp} \rightarrow\left(\Sigma_{\gamma\left(t_{k+1}\right)} X_{1}\right)^{\perp}$. Composing them, we get an isometry

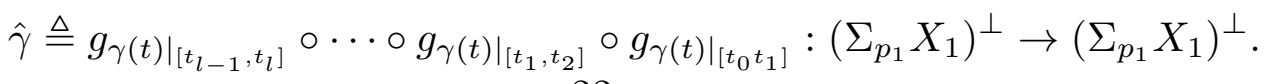


It is easy to see that $\hat{\gamma}\left(\left(\uparrow p_{p_{1}}\right)_{1}\right)=\left(\uparrow \uparrow_{p_{1}}\right)_{2}$, and $\hat{\gamma}\left(\uparrow p_{p_{1}}^{p_{2}^{\prime}}\right) \in \uparrow_{p_{1}}^{p_{2}^{\prime}}$ for any $\uparrow_{p_{1}}^{p_{2}^{\prime}}$ with $p_{2}^{\prime} \in X_{2}$. Moreover, due to Remark 4.3, it is not hard to see that there are neighborhoods $U_{p_{2}}, U_{\left(\uparrow_{\left.p_{1}\right)_{1}}^{p_{2}}\right.}$ and $U_{\left(\uparrow_{p_{1}}^{\left.p_{2}\right)_{2}}\right.}$ of $p_{2},\left(\uparrow_{p_{1}}^{p_{2}}\right)_{1}$ and $\left(\uparrow_{p_{1}}^{p_{2}}\right)_{2}$ respectively such that the following diagram commutes

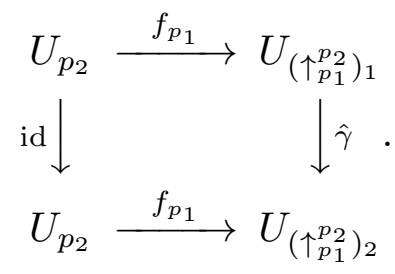

Hence, $\hat{\gamma}$ does not depend on the choice of $\left\{t_{k}\right\}_{k=0}^{l}$. (In fact, for all piecewise geodesics $\left.\tilde{\gamma}(t)\right|_{t \in[0,1]} \subset\left(\Sigma_{p_{2}} X_{2}\right)^{\perp}$ between $\left(\uparrow_{p_{2}}^{p_{1}}\right)_{1}$ and $\left(\uparrow_{p_{2}}^{p_{1}}\right)_{2}$ with $f_{p_{2}}^{-1}(\tilde{\gamma}(t)) \in X_{1}^{m}$, the isometries of $\left(\Sigma_{p_{1}} X_{1}\right)^{\perp}$ gotten through such an way are the same one.)

Case 2: There is $t_{0} \in(0,1)$ such that $\gamma\left(t_{0}\right) \notin X_{1}^{m}$, and there is no other $\left[p_{2} p_{1}\right]$ such that $\left|\uparrow_{p_{2}}^{p_{1}} \tilde{\gamma}\left(t_{0}\right)\right| \leq\left|\left(\uparrow_{p_{2}}^{p_{1}}\right)_{j} \tilde{\gamma}\left(t_{0}\right)\right|$ for $j=1$ and 2. In this case, by Lemma 4.6 (and its proof), it is not hard to see that both $\left.\gamma(t)\right|_{\left[0, t_{0}\right]}$ and $\left.\gamma(t)\right|_{\left[t_{0}, 1\right]}$ are a same minimal geodesic between $p_{1}$ and $\gamma\left(t_{0}\right)$, denoted by $\left[p_{1} \gamma\left(t_{0}\right)\right]$, and that there is an open and dense subset $A$ in $\left(\Sigma_{\gamma\left(t_{0}\right)} X_{1}\right)^{\perp}$ such that

$$
\lambda_{\uparrow_{\gamma\left(t_{0}\right)}^{p_{1}}} \zeta=2 \forall \zeta \in A \text { and } \lambda_{\uparrow_{\gamma\left(t_{0}\right)}^{p_{1}}} \zeta^{\prime} \leq 2 \forall \zeta^{\prime} \in\left(\Sigma_{\gamma\left(t_{0}\right)} X_{1}\right)^{\perp} \text {. }
$$

By Lemma 1.4 and (4.1.2), for any fixed $\left[p_{1} p_{2}^{\prime}\right]$ with $p_{2}^{\prime} \in X_{2}$, there is a unique $\left[\gamma\left(t_{0}\right) p_{2}^{\prime}\right]$ which together with $\left[p_{1} p_{2}^{\prime}\right]$ and $\left[p_{1} \gamma\left(t_{0}\right)\right]$ bounds a convex spherical surface $D$. Then (4.7) implies that, besides $\left[p_{1} p_{2}^{\prime}\right]$, there exists at most one $\left[p_{1} p_{2}^{\prime}\right]^{\prime}$ which together with $\left[\gamma\left(t_{0}\right) p_{2}^{\prime}\right]$ and $\left[p_{1} \gamma\left(t_{0}\right)\right]$ bounds a convex spherical surface $D^{\prime}$. Then we can define a 1-1 map

$$
\hat{\gamma}:\left(\Sigma_{p_{1}} X_{1}\right)^{\perp} \rightarrow\left(\Sigma_{p_{1}} X_{1}\right)^{\perp} \text { by } \uparrow p_{1}^{p_{2}^{\prime}} \mapsto\left(\uparrow p_{1}^{p_{2}^{\prime}}\right)^{\prime} \text { and }\left(\uparrow p_{1}^{p_{2}^{\prime}}\right)^{\prime} \mapsto \uparrow p_{1}^{p_{2}^{\prime}}
$$

where $\left(\uparrow_{p_{1}}^{p_{2}^{\prime}}\right)^{\prime}=\uparrow_{p_{1}}^{p_{2}^{\prime}}$ if $\left[p_{1} p_{2}^{\prime}\right]^{\prime}$ does not exist. Note that $\hat{\gamma}\left(\left(\uparrow p_{p_{1}}\right)_{1}\right)=\left(\uparrow_{p_{1}}^{p_{2}}\right)_{2}$ and $\hat{\gamma}\left(\uparrow \uparrow_{1}^{p_{2}^{\prime}}\right) \in \uparrow_{p_{1}}^{p_{2}^{\prime}}$. Similar to the proof of Lemma 4.2, we will conclude that $\hat{\gamma}$ is an isometry once we show that it is a continuous map. Hence, it suffices to show that $\left[p_{1} p_{2}^{j}\right]^{\prime} \rightarrow\left[p_{1} p_{2}^{\prime}\right]^{\prime}$ if $\left[p_{1} p_{2}^{j}\right] \rightarrow\left[p_{1} p_{2}^{\prime}\right]$ as $j \rightarrow \infty$ (where $\left.p_{2}^{j} \in X_{2}\right)$. Let $\left[\gamma\left(t_{0}\right) p_{2}^{j}\right]$ be the unique minimal geodesic which together with $\left[p_{1} p_{2}^{j}\right]$ (resp. $\left.\left[p_{1} p_{2}^{j}\right]^{\prime}\right)$ and $\left[p_{1} \gamma\left(t_{0}\right)\right]$ bounds a convex spherical surface $D_{j}\left(\operatorname{resp} . D_{j}^{\prime}\right)$. Note that $D_{j}\left(\right.$ resp. $\left.D_{j}^{\prime}\right)$ realizes a minimal geodesic $\left[\uparrow_{\gamma\left(t_{0}\right)}^{p_{2}^{j}} \uparrow_{\gamma\left(t_{0}\right)}^{p_{1}}\right]$ (resp. $\left.\left[\uparrow_{\gamma\left(t_{0}\right)}^{p_{2}^{j}} \uparrow_{\gamma\left(t_{0}\right)}^{p_{1}}\right]^{\prime}\right)$ in $\Sigma_{\gamma\left(t_{0}\right)} X$. Due to (4.1.2), we have that $D_{j} \rightarrow D$ as $j \rightarrow \infty$ because $\left[p_{1} p_{2}^{j}\right] \rightarrow\left[p_{1} p_{2}^{\prime}\right]$, so $\left[\uparrow_{\gamma\left(t_{0}\right)}^{p_{2}^{j}} \uparrow_{\gamma\left(t_{0}\right)}^{p_{1}}\right]$ converges to $\left[\uparrow_{\gamma\left(t_{0}\right)}^{p_{2}^{\prime}} \uparrow_{\gamma\left(t_{0}\right)}^{p_{1}}\right]$ which is realized by $D$. Then according to (4.7) and Lemma 1.4 on $\Sigma_{\gamma\left(t_{0}\right)} X,\left[\uparrow_{\gamma\left(t_{0}\right)}^{p_{2}^{j}} \uparrow_{\gamma\left(t_{0}\right)}^{p_{1}}\right]^{\prime}$ has to converge to $\left[\uparrow_{\gamma\left(t_{0}\right)}^{p_{2}^{\prime}} \uparrow_{\gamma\left(t_{0}\right)}^{p_{1}}\right]^{\prime}$ which is realized by $D^{\prime}$. It follows that $D_{j}^{\prime} \rightarrow D^{\prime}$ as $j \rightarrow \infty$, so $\left[p_{1} p_{2}^{j}\right]^{\prime} \rightarrow\left[p_{1} p_{2}^{\prime}\right]^{\prime}$. (Similarly, $\hat{\gamma}$ satisfies a corresponding diagram like (4.6).)

Case 3: There is $t_{0} \in(0,1)$ such that $\gamma\left(t_{0}\right) \notin X_{1}^{m}$, and there is a $\left[p_{2} p_{1}\right]_{3}$ such that $\left|\left(\uparrow_{p_{2}}^{p_{1}}\right)_{3} \tilde{\gamma}\left(t_{0}\right)\right| \leq\left|\left(\uparrow_{p_{2}}^{p_{1}}\right)_{j} \tilde{\gamma}\left(t_{0}\right)\right|$ for $j=1$ and 2. Since $\left.\tilde{\gamma}(t)\right|_{t \in[0,1]}$ is a minimal geodesic in $\left(\Sigma_{p_{2}} X_{2}\right)^{\perp}$ between $\left(\uparrow_{p_{2}}^{p_{1}}\right)_{1}$ and $\left(\uparrow_{p_{2}}^{p_{1}}\right)_{2}$, it has to hold that

$$
\left|\left(\uparrow_{p_{2}}^{p_{1}}\right)_{1}\left(\uparrow_{p_{2}}^{p_{1}}\right)_{3}\right|<\left|\left(\uparrow p_{p_{2}}^{p_{1}}\right)_{1}\left(\uparrow \uparrow_{p_{2}}^{p_{1}}\right)_{2}\right| \text { and }\left|\left(\uparrow_{p_{2}}^{p_{1}}\right)_{2}\left(\uparrow_{p_{2}}^{p_{1}}\right)_{3}\right|<\left|\left(\uparrow_{p_{2}}^{p_{1}}\right)_{1}\left(\uparrow_{p_{2}}^{p_{1}}\right)_{2}\right| \text {. }
$$


Then we can first look for the isometry of $\left(\Sigma_{p_{1}} X_{1}\right)^{\perp}$ determined by $\left[p_{1} p_{2}\right]_{1}$ and $\left[p_{1} p_{2}\right]_{3}$ (and $\left[p_{1} p_{2}\right]_{2}$ and $\left[p_{1} p_{2}\right]_{3}$ ) by repeating the above process. Since there are only $m$ pieces of minimal geodesics between $p_{1}$ and $p_{2}$, we can find the wanted isometry $\hat{\gamma}$ of $\left(\Sigma_{p_{1}} X_{1}\right)^{\perp}$, a composition of some isometries in Case 1 or 2 . Moreover, the $\hat{\gamma}$ satisfies a corresponding diagram like (4.6).

In the rest of this section, we will complete the proof of (A2) in Theorem A.

Proof of (A2) in Theorem A.

If $\operatorname{dim}\left(X_{1}\right)=0$ (or $\operatorname{dim}\left(X_{2}\right)=0$ ), then, by our convention, $\partial X_{1}=\emptyset$ means that $X_{1}$ consists of two points with distance $\pi$. This implies that $X=X_{1} * X_{2}$. From now on, we will assume that $\operatorname{dim}\left(X_{i}\right)>0$ for $i=1$ and 2 .

We first fix two arbitrary points $p_{1} \in X_{1}^{m}$ and $p_{2} \in X_{2}^{m}$, and let $\left\{\left[p_{1} p_{2}\right]_{k}\right\}_{k=1}^{m}$ be all minimal geodesics between $p_{1}$ and $p_{2}$. According to Lemma 4.4, $\left[p_{1} p_{2}\right]_{j}$ and $\left[p_{1} p_{2}\right]_{h}$ determines an isometry of $\left(\Sigma_{p_{1}} X_{1}\right)^{\perp}$, denoted by $\gamma_{j h}$, with $\gamma_{j h}\left(\left(\uparrow_{p_{1}}\right)_{j}\right)=$ $\left(\uparrow_{p_{1}}^{p_{2}}\right)_{h}$. And from the proof of Lemma 4.4 (especially by (4.6)), we know that

$$
\gamma_{h l} \cdot \gamma_{j h}=\gamma_{j l}
$$

and because $\gamma_{j h}\left(\left(\uparrow_{p_{1}}^{p_{2}}\right)_{1}\right)=\left(\uparrow_{p_{1}}^{p_{2}}\right)_{k}$ for some $k$, we have that $\gamma_{j h}=\gamma_{1 k}$. Hence,

$$
\Gamma_{2} \triangleq\left\{\gamma_{11}, \gamma_{12}, \cdots, \gamma_{1 m}\right\}
$$

is a finite group (where $\gamma_{11}$ is the unit element) which acts on $\left(\Sigma_{p_{1}} X_{1}\right)^{\perp}$ by isometries; and the orbit of any $\uparrow_{p_{1}}^{p_{2}^{\prime}}$ (with $p_{2}^{\prime} \in X_{2}$ ) under this action is just $\uparrow_{p_{1}}^{p_{2}^{\prime}}$. Based on Lemma 1.4, it is easy to see that the following map

$$
\pi_{2}:\left(\Sigma_{p_{1}} X_{1}\right)^{\perp} / \Gamma_{2} \longrightarrow X_{2} \text { by } \Uparrow_{p_{1}}^{p_{2}^{\prime}} \longrightarrow p_{2}^{\prime}
$$

is an isometry.

Similarly, $\left\{\left[p_{1} p_{2}\right]_{k}\right\}_{k=1}^{m}$ also determines a finite group

$$
\Gamma_{1} \triangleq\left\{\bar{\gamma}_{11}, \bar{\gamma}_{12}, \cdots, \bar{\gamma}_{1 m}\right\}
$$

which acts on $\left(\Sigma_{p_{2}} X_{2}\right)^{\perp}$ by isometries with $\bar{\gamma}_{j h}\left(\left(\uparrow p_{p_{2}}\right)_{j}\right)=\left(\uparrow_{p_{2}}^{p_{1}}\right)_{h}$; and

$$
\pi_{1}:\left(\Sigma_{p_{2}} X_{2}\right)^{\perp} / \Gamma_{1} \longrightarrow X_{1} \text { by } \Uparrow_{p_{2}}^{p_{1}^{\prime}} \longrightarrow p_{1}^{\prime}
$$

is also an isometry.

Claim 1: The map

$$
g: \Gamma_{2} \rightarrow \Gamma_{1} \text { defined by } \gamma_{1 k} \mapsto \bar{\gamma}_{1 k}^{-1}
$$

is an isomorphism. We need only to show that $g$ is a homomorphism, i.e.,

$$
g\left(\gamma_{1 k} \cdot \gamma_{1 k^{\prime}}\right)=g\left(\gamma_{1 k}\right) \cdot g\left(\gamma_{1 k^{\prime}}\right)
$$

Without loss of generality, due to the proof of Lemma 4.4, we can assume that $\gamma_{1 k}=\gamma_{1 k_{s}} \cdots \gamma_{1 k_{2}} \cdot \gamma_{1 k_{1}}$, and that each piecewise geodesic $\left.\gamma_{1 k_{j}}(t)\right|_{t \in[0,1]} \subset X_{1}$ (and $\left.\left.\tilde{\gamma}_{1 k_{j}}(t)\right|_{t \in[0,1]} \subset\left(\Sigma_{p_{2}} X_{2}\right)^{\perp}\right)$ determining $\gamma_{1 k_{j}}$ satisfies Case 1 or 2 in that proof. Note 
that $\gamma_{1 k}$ can be determined (like (4.5)) by the product of $\left.\gamma_{1 k_{1}}(t)\right|_{t \in[0,1]}, \cdots,\left.\gamma_{1 k_{s}}(t)\right|_{t \in[0,1]}$ (here, the product $\left.\alpha \circ \beta(t)\right|_{t \in[0,1]}$ of curves $\left.\alpha(t)\right|_{t \in[0,1]}$ and $\left.\beta(t)\right|_{t \in[0,1]}$ is defined by $\alpha \circ \beta(t)=\alpha(2 t)$ and $\beta(2 t-1)$ for $t \in\left[0, \frac{1}{2}\right]$ and $\left[\frac{1}{2}, 1\right]$ respectively).

Note that $\left.\tilde{\gamma}_{1 k_{j}}(t)\right|_{t \in[0,1]}$ is the unique minimal geodesic between $\left(\uparrow_{p_{2}}^{p_{1}}\right)_{1}$ and $\left(\uparrow_{p_{2}}\right)_{k_{j}}$ such that $f_{p_{2}}^{-1}\left(\left.\tilde{\gamma}_{1 k_{j}}(t)\right|_{t \in[0,1]}\right)=\left.\gamma_{1 k_{j}}(t)\right|_{t \in[0,1]}$ (see (3.2.1)). For convenience, we say that $\left.\tilde{\gamma}_{1 k_{j}}(t)\right|_{t \in[0,1]}$ is the lifting curve of $\left.\gamma_{1 k_{j}}(t)\right|_{t \in[0,1]}$ at $\left(\uparrow_{p_{2}}^{p_{1}}\right)_{1}$ in $\left(\Sigma_{p_{2}} X_{2}\right)^{\perp}$. Note that $\bar{\gamma}_{1 k_{j}}\left(\left(\uparrow p_{p_{2}}\right)_{1}\right)=\left(\uparrow p_{p_{2}}\right)_{k_{j}}$, so $\bar{\gamma}_{1 k_{j}}\left(\left(\uparrow_{p_{2}}\right)_{1}\right)$ is just $\tilde{\gamma}_{1 k_{j}}(1)$, the end point of $\left.\tilde{\gamma}_{1 k_{j}}(t)\right|_{t \in[0,1]}$. Furthermore, $\bar{\gamma}_{1 k_{1}}\left(\cdots\left(\bar{\gamma}_{1 k_{s}}\left(\left(\uparrow_{p_{2}}^{p_{1}}\right)_{1}\right)\right)\right)$ is the end point of the product of the lifting curves of $\left.\gamma_{1 k_{1}}(t)\right|_{t \in[0,1]}$ at $\left(\uparrow_{p_{2}}^{p_{1}}\right)_{1},\left.\gamma_{1 k_{2}}(t)\right|_{t \in[0,1]}$ at $\bar{\gamma}_{1 k_{1}}\left(\left(\uparrow_{p_{2}}^{p_{1}}\right)_{1}\right), \cdots$, and $\left.\gamma_{1 k_{s}}(t)\right|_{t \in[0,1]}$ at $\bar{\gamma}_{1 k_{1}}\left(\cdots\left(\bar{\gamma}_{1 k_{s-1}}\left(\left(\uparrow_{p_{2}}\right)_{1}\right)\right)\right)$ (note that this product is also the lifting curve at $\left(\uparrow_{p_{2}}^{p_{1}}\right)_{1}$ of the product of $\left.\gamma_{1 k_{1}}(t)\right|_{t \in[0,1]}, \cdots$, and $\left.\left.\gamma_{1 k_{s}}(t)\right|_{t \in[0,1]}\right)$. This implies that

$$
\bar{\gamma}_{1 k_{1}} \cdot \cdots \cdot \bar{\gamma}_{1 k_{s}}=\bar{\gamma}_{1 k} .
$$

It follows that

$$
g\left(\gamma_{1 k}\right)=\bar{\gamma}_{1 k}^{-1}=\bar{\gamma}_{1 k_{s}}^{-1} \cdot \cdots \cdot \bar{\gamma}_{1 k_{1}}^{-1}=g\left(\gamma_{1 k_{s}}\right) \cdot \cdots \cdot g\left(\gamma_{1 k_{1}}\right)
$$

which implies that (4.10).

Due to Claim 1, we let $\Gamma \triangleq\left\{\gamma_{1}, \gamma_{2}, \cdots, \gamma_{m}\right\}$ denote $\Gamma_{1}$ and $\Gamma_{2}$, and let $\Gamma$ act on $\left(\Sigma_{p_{i}} X_{i}\right)^{\perp}$ by isometries such that $\gamma_{k}\left(q_{2}\right)=\gamma_{1 k}\left(q_{2}\right)$ for any $q_{2} \in\left(\Sigma_{p_{1}} X_{1}\right)^{\perp}$ and $\gamma_{k}\left(q_{1}\right)=\bar{\gamma}_{1 k}^{-1}\left(q_{1}\right)$ for any $q_{1} \in\left(\Sigma_{p_{2}} X_{2}\right)^{\perp}$. Then the $\Gamma$-action on $\left(\Sigma_{p_{i}} X_{i}\right)^{\perp}$ extends uniquely to an isometric $\Gamma$-action on $\left(\Sigma_{p_{1}} X_{1}\right)^{\perp} *\left(\Sigma_{p_{2}} X_{2}\right)^{\perp}$. Next, through the following steps, we will construct a 1-1 map

$$
\pi:\left(\left(\Sigma_{p_{1}} X_{1}\right)^{\perp} *\left(\Sigma_{p_{2}} X_{2}\right)^{\perp}\right) / \Gamma \rightarrow X
$$

For convenience, let $\bar{X}$ denote $\left(\left(\Sigma_{p_{1}} X_{1}\right)^{\perp} *\left(\Sigma_{p_{2}} X_{2}\right)^{\perp}\right) / \Gamma$, and let $\bar{X}_{j}$ denote $\left(\Sigma_{p_{i}} X_{i}\right)^{\perp} / \Gamma \subset \bar{X}(j \neq i \in\{1,2\})$, and let $\bar{p}_{j}^{\prime}$ denote any $\Uparrow_{p_{i}}^{p_{j}^{\prime}} \in \bar{X}_{j}$.

Step 1: Define $\pi\left(\bar{p}_{i}^{\prime}\right)=p_{i}^{\prime}$ for any $\bar{p}_{i}^{\prime} \in \bar{X}_{i} \subset \bar{X}$, i.e., $\left.\pi\right|_{\bar{X}_{i}}=\pi_{i}$.

Step 2: We define $\pi\left(\left[\bar{p}_{1} \bar{p}_{2}^{\prime}\right]\right)$ for all $\left[\bar{p}_{1} \bar{p}_{2}^{\prime}\right]$. Note that there is a natural isometry

$$
i_{\bar{p}_{i}}:\left(\Sigma_{\bar{p}_{i}} \bar{X}_{i}\right)^{\perp} \rightarrow\left(\Sigma_{p_{i}} X_{i}\right)^{\perp}
$$

(where $\left(\Sigma_{\bar{p}_{i}} \bar{X}_{i}\right)^{\perp}$ belongs to $\left.\Sigma_{\bar{p}_{i}} \bar{X}\right)$. Then we can define $\pi\left(\left[\bar{p}_{1} \bar{p}_{2}^{\prime}\right]\right)$ to be $\left[p_{1} p_{2}^{\prime}\right]^{7}$ (a minimal geodesics between $\pi\left(\bar{p}_{1}\right)$ and $\left.\pi\left(\bar{p}_{2}^{\prime}\right)\right)$ such that $\uparrow_{p_{1}}^{p_{2}}=i_{\bar{p}_{1}}\left(\uparrow \bar{p}_{1}\right)$.

Step 3: For any $\bar{p}_{1}^{\prime} \in \bar{X}_{1}$ with $\left(\pi\left(\bar{p}_{1}^{\prime}\right)=\right) p_{1}^{\prime} \in X_{1}^{m}$, we will define $\pi\left(\left[\bar{p}_{1}^{\prime} \bar{p}_{2}^{\prime}\right]\right)$ for all $\left[\bar{p}_{1}^{\prime} \bar{p}_{2}^{\prime}\right]$ with $\bar{p}_{2}^{\prime} \in \bar{X}_{2}$. Select $\left[\bar{p}_{1} \bar{p}_{1}^{\prime}\right] \subset \bar{X}_{1}$ and $\left[p_{1} p_{1}^{\prime}\right] \subset X_{1}$ such that $\pi\left(\left[\bar{p}_{1} \bar{p}_{1}^{\prime}\right]\right)=$ $\left[p_{1} p_{1}^{\prime}\right]$. Based on Lemma 4.2 and (4.11), we can construct an isometry

$$
i_{\bar{p}_{1}^{\prime}, p_{1}} \triangleq h_{\left[p_{1} p_{1}^{\prime}\right]} \circ i_{\bar{p}_{1}} \circ h_{\left[\bar{p}_{1}^{\prime} \bar{p}_{1}\right]}:\left(\Sigma_{\bar{p}_{1}^{\prime}} \bar{X}_{1}\right)^{\perp} \rightarrow\left(\Sigma_{p_{1}^{\prime}} X_{1}\right)^{\perp}
$$

Claim 2: $i_{\bar{p}_{1}^{\prime}, p_{1}}$ does not depend the choice of $\left[p_{1} p_{1}^{\prime}\right] \subset X_{1}$ (and $\left[\bar{p}_{1} \bar{p}_{1}^{\prime}\right] \subset \bar{X}_{1}$ ). By

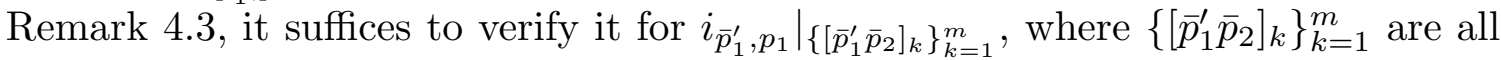
minimal geodesics between $\bar{p}_{1}^{\prime}$ and $\bar{p}_{2}$. In fact, this is clear due to the definition of

\footnotetext{
${ }^{7}$ In the present paper, " $\pi\left(\left[\bar{p}_{1} \bar{p}_{2}^{\prime}\right]\right)$ is defined to be $\left[p_{1} p_{2}^{\prime}\right]$ " means that, for any $\bar{x} \in\left[\bar{p}_{1} \bar{p}_{2}^{\prime}\right], \pi(\bar{x})$ is defined to be $x \in\left[p_{1} p_{2}^{\prime}\right]$ with $\left|x p_{1}\right|=\left|\bar{x} \bar{p}_{1}\right|$.
} 
$\pi_{1}\left(=\left.\pi\right|_{\bar{X}_{1}}\right)$ and the isometry $i_{\bar{p}_{2}}:\left(\Sigma_{\bar{p}_{2}} \bar{X}_{2}\right)^{\perp} \rightarrow\left(\Sigma_{p_{2}} X_{2}\right)^{\perp}$ (see (4.11)). Based on Claim 2, we can similarly define $\pi\left(\left[\bar{p}_{1}^{\prime} \bar{p}_{2}^{\prime}\right]\right)$ to be $\left[p_{1}^{\prime} p_{2}^{\prime}\right]$ such that $\uparrow_{p_{1}^{\prime}}^{p_{2}^{\prime}}=i_{\bar{p}_{1}^{\prime}, p_{1}}\left(\uparrow_{\bar{p}_{1}^{\prime}}^{\bar{p}_{2}^{\prime}}\right)$.

Step 4: We will define $\pi\left(\left[\bar{p}_{1}^{\prime} \bar{p}_{2}^{\prime}\right]\right)$ for all $\left[\bar{p}_{1}^{\prime} \bar{p}_{2}^{\prime}\right]$ with $\left(\pi\left(\bar{p}_{1}^{\prime}\right)=\right) p_{1}^{\prime} \notin X_{1}^{m}$ and $\bar{p}_{2}^{\prime} \in \bar{X}_{2}$. Since $\pi_{1}$ is an isometry (see (4.9)), each element of $\Uparrow_{p_{2}}^{p_{1}^{\prime}} \subset\left(\Sigma_{p_{2}} X_{2}\right)^{\perp}$ has an isotropy subgroup $\Gamma_{\bar{p}_{1}^{\prime}}$ with $\left|\Gamma_{\bar{p}_{1}^{\prime}}\right|=\frac{m}{m_{p_{1}^{\prime}}}$ (with respect to the $\Gamma$-action on $\left.\left(\Sigma_{p_{2}} X_{2}\right)^{\perp}\right)$. It then follows that

$$
\left(\Sigma_{\bar{p}_{1}^{\prime}} \bar{X}_{1}\right)^{\perp}=\left(\Sigma_{\bar{p}_{1}} \bar{X}_{1}\right)^{\perp} / \Gamma_{\bar{p}_{1}^{\prime}}=\left(\Sigma_{p_{1}} X_{1}\right)^{\perp} / \Gamma_{\bar{p}_{1}^{\prime}}
$$

On the other hand, we have the following cone-neighborhood isometry chain

$$
X_{2} \stackrel{f_{p_{1}^{\prime}}}{\longrightarrow}\left(\Sigma_{p_{1}^{\prime}} X_{1}\right)^{\perp} \stackrel{f_{\uparrow_{p}^{p_{1}}}}{\longrightarrow}\left(\Sigma_{\uparrow_{p_{1}^{\prime}}^{p_{1}}}\left(\Sigma_{p_{1}^{\prime}} X_{1}\right)\right)^{\perp}
$$

(see (4.1) for $f_{p_{1}^{\prime}}$ ), where $f_{\uparrow_{p_{1}^{\prime}}^{p_{1}}}$ is the corresponding map to (4.1) for a given $\left[p_{1}^{\prime} p_{1}\right] \subset$ $X_{1}$ in considering $\Sigma_{p_{1}^{\prime}} X\left(\supset \Sigma_{p_{1}^{\prime}} X_{1},\left(\Sigma_{p_{1}^{\prime}} X_{1}\right)^{\perp}\right)$. Then, similar to $h_{\left[p_{1} p_{1}^{\prime}\right]}$ in Lemma 4.2 , we can define a natural isometry

$$
h_{\left[p_{1} p_{1}^{\prime}\right]}:\left(\Sigma_{p_{1}} X_{1}\right)^{\perp} \rightarrow\left(\Sigma_{\uparrow_{p_{1}^{\prime}}^{p_{1}}}\left(\Sigma_{p_{1}^{\prime}} X_{1}\right)\right)^{\perp}
$$

(Hint: by Lemma 4.5 (and its proof), $\left\{\left[\uparrow_{p_{1}^{\prime} p_{1}^{p_{1}^{\prime}}}^{p_{2}^{\prime}}\right] \subset \Sigma_{p_{1}^{\prime}} X \mid \uparrow_{p_{1}^{\prime}}^{p_{2}^{\prime}} \in\left(\Sigma_{p_{1}^{\prime}} X_{1}\right)^{\perp}\right\}$ is mapped $1-1$ to $\left.\left\{\left[p_{1} p_{2}^{\prime}\right] \subset X \mid p_{2}^{\prime} \in X_{2}\right\}\right)$. Moreover, similar to (4.9), there is a finite group $\Gamma_{p_{1}^{\prime}}$ such that

$$
\left(\Sigma_{p_{1}^{\prime}} X_{1}\right)^{\perp}=\left(\Sigma_{\uparrow_{p_{1}^{\prime}}^{p_{1}}}\left(\Sigma_{p_{1}^{\prime}} X_{1}\right)\right)^{\perp} / \Gamma_{p_{1}^{\prime}}=\left(\Sigma_{p_{1}} X_{1}\right)^{\perp} / \Gamma_{p_{1}^{\prime}}
$$

Based on Lemma 4.6 (and its proof), it is not hard to conclude that

$$
\Gamma_{\bar{p}_{1}^{\prime}}=\Gamma_{p_{1}^{\prime}}
$$

Therefore, (4.13) and (4.14) enable us to construct a natural isometry from $\left(\Sigma_{\bar{p}_{1}^{\prime}} \bar{X}_{1}\right)^{\perp}$ to $\left(\Sigma_{p_{1}^{\prime}} X_{1}\right)^{\perp}$, which is similar to $(4.12)$ and does not depend the choice of $\left[p_{1} p_{1}^{\prime}\right]$. Then we can similarly define $\pi\left(\left[\bar{p}_{1}^{\prime} \bar{p}_{2}^{\prime}\right]\right)$.

So far, we have finished the definition of $\pi$, which is a 1-1 map. We need to show that it is an isometry, i.e.,

$$
|\pi(x) \pi(y)|=|x y| \text { for all } x, y \in\left(\left(\Sigma_{p_{1}} X_{1}\right)^{\perp} *\left(\Sigma_{p_{2}} X_{2}\right)^{\perp}\right) / \Gamma \text {. }
$$

Claim 3: $\pi$ is a continuous map, so it is a homeomorphism. It suffices to show that $\pi\left(\left[\bar{p}_{1}^{j} \bar{p}_{2}^{j}\right]\right) \rightarrow \pi\left(\left[\bar{p}_{1}^{\prime} \bar{p}_{2}^{\prime}\right]\right)$ if $\left[\bar{p}_{1}^{j} \bar{p}_{2}^{j}\right] \rightarrow\left[\bar{p}_{1}^{\prime} \bar{p}_{2}^{\prime}\right]$ as $j \rightarrow \infty$. We select $\left[\bar{p}_{1} \bar{p}_{1}^{j}\right],\left[\bar{p}_{1} \bar{p}_{1}^{\prime}\right] \subset \bar{X}_{1}$ such that $\left[\bar{p}_{1} \bar{p}_{1}^{j}\right] \rightarrow\left[\bar{p}_{1} \bar{p}_{1}^{\prime}\right]$. By Lemma 1.4 , we can find $\left[\bar{p}_{1} \bar{p}_{2}^{j}\right]$ and $\left[\bar{p}_{1} \bar{p}_{2}^{\prime}\right]$ such that $\left[\bar{p}_{1} \bar{p}_{1}^{j}\right],\left[\bar{p}_{1}^{j} \bar{p}_{2}^{j}\right]$ and $\left[\bar{p}_{1} \bar{p}_{2}^{j}\right]$ (resp. $\left[\bar{p}_{1} \bar{p}_{1}^{\prime}\right],\left[\bar{p}_{1}^{\prime} \bar{p}_{2}^{\prime}\right]$ and $\left[\bar{p}_{1} \bar{p}_{2}^{\prime}\right]$ ) bound a convex spherical surface $D_{j}$ (resp. $D$ ), and $D_{j} \rightarrow D$ (of course, $\left[\bar{p}_{1} \bar{p}_{2}^{j}\right] \rightarrow\left[\bar{p}_{1} \bar{p}_{2}^{\prime}\right]$ ). From the definition of $\pi$, it is not hard to see that $\pi\left(D_{j}\right)$ (resp. $\left.\pi(D)\right)$ is the convex spherical surface bounded by $\pi\left(\left[\bar{p}_{1} \bar{p}_{1}^{j}\right]\right), \pi\left(\left[\bar{p}_{1}^{j} \bar{p}_{2}^{j}\right]\right)$ and $\pi\left(\left[\bar{p}_{1} \bar{p}_{2}^{j}\right]\right)$ (resp. $\pi\left(\left[\bar{p}_{1} \bar{p}_{1}^{\prime}\right]\right), \pi\left(\left[\bar{p}_{1}^{\prime} \bar{p}_{2}^{\prime}\right]\right)$ and 
$\left.\pi\left(\left[\bar{p}_{1} \bar{p}_{2}^{\prime}\right]\right)\right)$, and that $\pi\left(\left[\bar{p}_{1} \bar{p}_{1}^{j}\right]\right) \rightarrow \pi\left(\left[\bar{p}_{1} \bar{p}_{1}^{\prime}\right]\right)$ and $\pi\left(\left[\bar{p}_{1} \bar{p}_{2}^{j}\right]\right) \rightarrow \pi\left(\left[\bar{p}_{1} \bar{p}_{2}^{\prime}\right]\right)$. Note that $\pi\left(D_{j}\right)$ converges to a convex spherical surface including $\pi\left(\left[\bar{p}_{1} \bar{p}_{1}^{\prime}\right]\right)$ and $\pi\left(\left[\bar{p}_{1} \bar{p}_{2}^{\prime}\right]\right)$. Due to (4.1.2), it has to hold that $\pi\left(D_{j}\right) \rightarrow \pi(D)$ as $j \rightarrow \infty$, so $\pi\left(\left[\bar{p}_{1}^{j} \bar{p}_{2}^{j}\right]\right) \rightarrow \pi\left(\left[\bar{p}_{1}^{\prime} \bar{p}_{2}^{\prime}\right]\right)$.

By Claim 3 and Remark 2.4, for any $[x y], \pi([x y])$ is a continuous curve with length equal to that of $[x y]$ (note that the projection of $[x y]$ on $\bar{X}_{i}$ is a piecewise geodesic, which is mapped by $\pi$ to the projection of $\pi([x y])$ on $X_{i}$ ), which implies

$$
|\pi(x) \pi(y)| \leq|x y| .
$$

Similarly, by considering $\pi^{-1}$, we can conclude that

$$
|x y| \leq|\pi(x) \pi(y)|
$$

which implies (4.15).

\section{Proof of Corollary 0.2 And 0.3}

Proof of Corollary 0.2.

Since $M$ and $M_{i}$ are all Riemannian manifolds, for any $p_{i} \in M_{i}$, each $\left(\Sigma_{p_{i}} M_{i}\right)^{\perp}$ is a unit sphere, so is $\left(\Sigma_{p_{1}} M_{1}\right)^{\perp} *\left(\Sigma_{p_{2}} M_{2}\right)^{\perp}$. Then Corollary 0.2 is a corollary of (A2) except when one of $\operatorname{dim}\left(M_{i}\right)$ is equal to 0 (note that in (A2), if $\operatorname{dim}\left(X_{i}\right)=0$, then $X_{i}$ consists of two points with distance $\pi$, while in Corollary 0.2 , if $\operatorname{dim}\left(M_{i}\right)=0$, then $M_{i}$ is a single point). Hence, we need only to consider the case where $\operatorname{dim}\left(M_{1}\right)=0$ (or $\operatorname{dim}\left(M_{2}\right)=0$ ), i.e. $M_{1}=\left\{p_{1}\right\}$. By Lemma 2.1, we have that $m=1$ or 2 . And since $M$ is a Riemannian manifold, then it is not hard to see that $M_{2}^{m}=M_{2}$ (see Remark 5.1 below). By (2.3.2), we can conclude that there is an $m$-order Riemannian cover from $\Sigma_{p_{1}} M\left(=\mathbb{S}^{n-1}\right)$ to $M_{2}$. If $m=1$, then $M_{2}$ is isometric to a unit sphere, so is $M$. If $m=2$, then it is easy see that $p \in M$ belongs to some $\left[p_{1} p_{2}\right]$ with $p_{2} \in M_{2}$. It then follows from Lemma 1.4 and (2.3.2) that $\sec _{M} \equiv 1$, so $M$ is isometric to $\mathbb{R} \mathbb{P}^{n}$.

Remark 5.1. We would like to point out that in the case $X$ is a Riemannian manifold, our proof can be made direct and simple, e.g., discussions in Section 3 and 4 are not required. Precisely, since $\Sigma_{p_{i}} M_{i}$ is a unit sphere, $M_{i}^{m}=M_{i}$, and a local join structure from (2.3.2) and (2.3.3) implies that $\sec _{M} \equiv 1$.

\section{Proof of Corollary 0.3.}

Let $M=\mathbb{S}^{n} / \Gamma$, where $\mathbb{S}^{n}$ is the unit sphere. If $\Gamma=\langle\gamma\rangle$, then $\gamma$ is represented by a closed geodesic on $M / \Gamma$, and $\gamma$ preserves a great circle $S^{1}$. Clearly, $\Gamma$ also preserves the sphere $\mathbb{S}^{n-2}=\left(S^{1}\right)=\frac{\pi}{2}$. It then follows that $M_{1}=S^{1} / \Gamma$ and $M_{2}=\mathbb{S}^{n-2} / \Gamma$ satisfy the conditions of Corollary 0.2.

In general, $\Gamma$ contains a normal cyclic subgroup $\mathbb{Z}_{q}$ with $\left[\Gamma: \mathbb{Z}_{q}\right] \leq w(n)$, a constant depending only on $n$ ([Ro]). By the above, the normal covering space of $M, \mathbb{S}^{n} / \mathbb{Z}_{q}$, satisfies Corollary 0.2 .

Remark 5.2. From the above proof, we also see the following: if a spherical space form has diameter less than $\frac{\pi}{2}$, then its fundamental group is not cyclic. 


\section{Proof of Theorem B}

The approach to Theorem $B$ is similar to that in the proof of (A2). The main difference is in showing that for $p_{2} \in \partial X_{2}, f_{p_{2}}\left(X_{1}\right)=\Uparrow_{p_{2}}^{X_{1}}=\left(\Sigma_{p_{2}} X_{2}\right)=\frac{\pi}{2}$ (which guarantees that any $x \in X$ belongs to some $\left[p_{1}^{\prime} p_{2}^{\prime}\right]$ with $\left.p_{i}^{\prime} \in X_{i}\right)$, and for any $p_{1} \in X_{1}, f_{p_{1}}\left(X_{2}\right)=\Uparrow_{p_{1}}^{X_{2}}=\left(\Sigma_{p_{1}} X_{1}\right)=\frac{\pi}{2}$ (here, $\left.\partial X_{2} \neq \emptyset\right)$.

For convenience, we list again the main conditions of Theorem B:

$\operatorname{dim}\left(X_{1}\right)+\operatorname{dim}\left(X_{2}\right)=n-1, X_{2}^{=\frac{\pi}{2}}=X_{2}^{\geq \frac{\pi}{2}},\left(\Sigma_{p_{2}} X_{2}\right)^{=\frac{\pi}{2}}=\left(\Sigma_{p_{2}} X_{2}\right)^{\geq \frac{\pi}{2}}$ for $p_{2} \in \partial X_{2}$.

Let's first give a (generalized) version of Key Lemma 1.6 at points in $\partial X_{2}$.

Lemma 6.1. For any $p_{2} \in \partial X_{2}$, there is an $m_{p_{2}} \leq m$ (where $m$ is the number in Key Lemma 1.6) such that $\lambda_{p_{1} p_{2}} \leq m_{p_{2}}$ for all $p_{1} \in X_{1}$, and $\left\{p_{1} \in X_{1} \mid \lambda_{p_{1} p_{2}}=m_{p_{2}}\right\}$ is open and dense in $X_{1}$.

Proof. By Lemma 1.2 and the first variation formula, for a given $\left[p_{2} p_{1}\right],\left|\uparrow_{p_{2}}^{p_{1}} \xi\right| \geq \frac{\pi}{2}$ for all $\xi \in \Sigma_{p_{2}} X_{2}$. Since $\left(\Sigma_{p_{2}} X_{2}\right)^{\geq \frac{\pi}{2}}=\left(\Sigma_{p_{2}} X_{2}\right)^{=\frac{\pi}{2}}$, we in fact have that

$$
\left|\uparrow_{p_{2}}^{p_{1}} \xi\right|=\frac{\pi}{2}
$$

Then for any $\left[p_{2} p_{2}^{\prime}\right] \subseteq X_{2}$, there is a $\left[p_{2}^{\prime} p_{1}\right]$ which together with $\left[p_{2} p_{1}\right]$ and $\left[p_{2} p_{2}^{\prime}\right]$ bounds a convex spherical surface (by (1.1.3)). On the other hand, since $X_{1}$ has empty boundary, for any $p_{2}^{\prime} \in X_{2}^{m}$ (ref. Key Lemma 1.6), we still have that $\Sigma_{p_{2}^{\prime}} X=\Sigma_{p_{2}^{\prime}} X_{2} *\left(\Sigma_{p_{2}^{\prime}} X_{2}\right)^{\perp}($ see $(4.1 .2))$. It then follows that

$$
\lambda_{p_{1} p_{2}} \leq \lambda_{p_{1} p_{2}^{\prime}} \leq m
$$

Then similar to Lemma 2.6, for any $\left[p_{1} p_{1}^{\prime}\right] \subset X_{1}$, we can conclude that $\lambda_{p_{1}^{\prime \prime} p_{2}}$ is a constant for all $p_{1}^{\prime \prime} \in\left[p_{1} p_{1}^{\prime}\right]^{\circ}$, and $\lambda_{p_{1} p_{2}}, \lambda_{p_{1}^{\prime} p_{2}} \leq \lambda_{p_{1}^{\prime \prime} p_{2}}$. This together with Lemma 2.2 implies that there is an $m_{p_{2}} \leq m$ such that $\lambda_{p_{1} p_{2}} \leq m_{p_{2}}$ for all $p_{1} \in X_{1}$, and $\left\{p_{1} \in X_{1} \mid \lambda_{p_{1} p_{2}}=m_{p_{2}}\right\}$ is open and dense in $X_{1}$. (However, so far we do not know that $X_{1}^{m}$ belongs to $\left\{p_{1} \in X_{1} \mid \lambda_{p_{1} p_{2}}=m_{p_{2}}\right\}$.)

Based on Lemma 6.1, using the proof of Key Lemma 1.7, we can conclude a version of Key Lemma 1.7 at points in $\partial X_{2}$.

Lemma 6.2. For any $p_{2} \in \partial X_{2}, f_{p_{2}}: X_{1} \rightarrow\left(\Sigma_{p_{2}} X_{2}\right)=\frac{\pi}{2}$ is also a cone-neighborhood isometry. As a result, we have that $\Uparrow_{p_{2}}^{X_{1}}=\left(\Sigma_{p_{2}} X_{2}\right)=\frac{\pi}{2}$, which has an empty boundary.

Corollary 6.3. For any $x \in X$, there is a $\left[p_{1} p_{2}\right]$ with $p_{i} \in X_{i}$ such that $x \in\left[p_{1} p_{2}\right]$. Proof. Let $p_{2} \in X_{2}$ such that $\left|x p_{2}\right|=\min \left\{\left|x p_{2}^{\prime}\right| \mid p_{2}^{\prime} \in X_{2}\right\}$. Note that $\left|x p_{2}\right| \leq \frac{\pi}{2}$ because $X_{2}^{\geq \frac{\pi}{2}}=X_{2}^{=\frac{\pi}{2}}$. By a similar proof of (6.1) for $p_{2} \in \partial X_{2}$ or by Lemma 1.3 for $p_{2} \in X_{2}^{\circ}$, we know that $\uparrow_{p_{2}}^{x} \in\left(\Sigma_{p_{2}} X_{2}\right)=\frac{\pi}{2}$ for any $\left[p_{2} x\right]$. On the other hand, by Lemma 6.2 or Key Lemma 1.7, $\Uparrow_{p_{2}}^{X_{1}}=\left(\Sigma_{p_{2}} X_{2}\right)=\frac{\pi}{2}$ for any $p_{2} \in X_{2}$. It then follows that there exists $\left[p_{2} p_{1}\right]$ with $p_{1} \in X_{1}$ such that $\uparrow_{p_{2}}^{x}=\uparrow_{p_{2}}^{p_{1}}$, which implies that $\left[p_{2} x\right] \subseteq\left[p_{2} p_{1}\right]$.

Based on Corollary 6.3, we can give a generalized version of Key Lemma 1.7 at points in $X_{1}$ (compared to Theorem A, $\partial X_{2} \neq \emptyset$ here). 
Lemma 6.4. (6.4.1) For any $p_{1} \in X_{1}$, $\Uparrow_{p_{1}}^{X_{2}}=\left(\Sigma_{p_{1}} X_{1}\right)^{\perp}$; and $f_{p_{1}}: X_{2} \rightarrow\left(\Sigma_{p_{1}} X_{1}\right)^{\perp}$ is also a cone-neighborhood isometry.

(6.4.2) For any $p_{1} \in X_{1}^{m}, \Sigma_{p_{1}} X=\Sigma_{p_{1}} X_{1} *\left(\Sigma_{p_{1}} X_{1}\right)^{\perp}$.

Proof of (6.4.1).

For the former part, by (1.1) we need only to show that, for any $\zeta \in\left(\Sigma_{p_{1}} X_{1}\right)^{\perp}$, there is a $\left[p_{1} p_{2}\right]$ with $p_{2} \in X_{2}$ such that $\zeta=\uparrow_{p_{1}}^{p_{2}}$. We first assume that there is a $\left[p_{1} x\right]$ such that $\zeta=\uparrow_{p_{1}}^{x}$. Note that we can assume that there is a unique minimal geodesic between $x$ and $p_{1}$ (otherwise we can select $x^{\prime} \in\left[p_{1} x\right]^{\circ}$ to take the place of $x$ ). By Corollary $6.3, x$ belongs to some $\left[p_{1}^{\prime} p_{2}^{\prime}\right]$ with $p_{i}^{\prime} \in X_{i}$. It suffices to show that $p_{1}^{\prime}=p_{1}$. If $p_{1}^{\prime} \neq p_{1}$, then by Lemma 1.4 there is a triangle $\triangle p_{1} p_{1}^{\prime} p_{2}^{\prime}$ containing $\left[p_{1}^{\prime} p_{2}^{\prime}\right]$ which bounds a convex spherical surface. Since there is a unique minimal geodesic between $x$ and $p_{1},\left[p_{1} x\right]$ has to lie in this surface. This implies that $\uparrow_{p_{1}}^{x}$ is not perpendicular to the side $\left[p_{1} p_{1}^{\prime}\right]\left(\subset X_{1}\right)$ in the $\triangle p_{1} p_{1}^{\prime} p_{2}^{\prime}$, which contradicts $\uparrow_{p_{1}}^{x} \in\left(\Sigma_{p_{1}} X_{1}\right)^{\perp}$. Next, we need only to show that there must be a $\left[p_{1} x\right]$ such that $\zeta=\uparrow_{p_{1}}^{x}$. If this is not true, we can find $\left[p_{1} x_{j}\right]$ such that $\uparrow_{p_{1}}^{x_{j}} \rightarrow \zeta$ as $j \rightarrow \infty$ ([BGP $\left.]\right)$. Using the standard limiting argument we can find a $\left[p_{1} p_{2}\right]$ with $p_{2} \in X_{2}$ such that $\zeta=\uparrow_{p_{1}}^{p_{2}}$, a contradiction.

For the latter part, we can refer to the proof for (3.9) (here, we need Lemma 6.1 in addition; and notice that, due to Lemma 6.2, Lemma 1.4 still holds even if $\left.p_{2} \in \partial X_{2}\right)$.

Given Lemma 6.2, Corollary 6.3 and (6.4.1), the proof of Lemma 4.5 will go through without a change. That is, we have the following.

Lemma 6.5. In the situation here, the statement in Lemma 4.5 is still true.

In order to prove (6.4.2), we need the following technical lemma.

Lemma 6.6. Let $A \in$ Alex $^{n}(1)$, and let $A_{1}, A_{2}$ be two compact convex subsets with $\partial A_{1}=\emptyset$ and $\left|A_{1} A_{2}\right| \geq \frac{\pi}{2}$. If $\lambda_{a_{1} a_{2}}<+\infty$ for all $a_{i} \in A_{i}$ and $\lambda_{a_{1} a_{2}}=1$ for $a_{2} \in A_{2}^{\circ}$ and $a_{1} \in A_{1}$, and if any $a \in A$ belongs to some $\left[a_{1} a_{2}\right]$ with $a_{i} \in A_{i}$, then we have that $A=A_{1} * A_{2}$.

Proof. By Lemma 1.3, $\left|a_{1} a_{2}\right|=\frac{\pi}{2}$ for all $a_{i} \in A_{i}$, so $A_{1} * A_{2}^{\circ}$ can be isometrically embedded into $A$. Hence, we need only consider the case where $\partial A_{2} \neq \emptyset$ and $\operatorname{dim}\left(A_{2}\right)>0$ (note that if $\operatorname{dim}\left(A_{2}\right)=0$, then it is our convention that $A_{2}=A_{2}^{\circ}$ ). Claim: For $a_{2} \in \partial A_{2}$, any $\left[a_{2} a_{1}\right]$ with $a_{1} \in A_{1}$ satisfies $\left|\uparrow_{a_{2}}^{a_{1}} \xi\right|=\frac{\pi}{2}$ for all $\xi \in \Sigma_{a_{2}} A_{2}$. Then for any $\left[a_{2} a_{2}^{\prime}\right] \subseteq A_{2}$ with $a_{2}^{\prime} \in A_{2}^{\circ}$, there is a $\left[a_{2}^{\prime} a_{1}\right]$ which together with $\left[a_{2} a_{1}\right]$ and $\left[a_{2} a_{2}^{\prime}\right]$ bounds a convex spherical surface (by (1.1.3)). Since any $a \in A$ belongs to some $\left[a_{1} a_{2}\right]$ with $a_{i} \in A_{i}$, this implies that

$$
A=\overline{A_{1} * A_{2}^{\circ}} \text {, the closure of } A_{1} * A_{2}^{\circ}
$$

(note that $\left[a_{2} a_{2}^{\prime}\right] \backslash\left\{a_{2}\right\} \subseteq A_{2}^{\circ}$ ). It therefore follows that $A=A_{1} * A_{2}$.

Next, we need only to verify the claim. Consider the multi-valued map

$$
f_{a_{2}}: A_{1} \rightarrow \Sigma_{a_{2}} A \text { defined by } a_{1} \rightarrow \Uparrow_{a_{2}}^{a_{1}}
$$

(Note that, by Theorem 1.8, we have that $\operatorname{dim}\left(A_{1}\right)<n-1=\operatorname{dim}\left(\Sigma_{a_{2}} A\right)$.) Since $\lambda_{a_{1} a_{2}}<+\infty$ for all $a_{1} \in A_{1}$, by Lemma 2.2 and 2.6 (like in the proof of Lemma 6.1) we can conclude that there is an $m$ such that $\lambda_{a_{1} a_{2}} \leq m$ for all $a_{1} \in A_{1}$ and 
$\left\{a_{1} \in A_{1} \mid \lambda_{a_{1} a_{2}} \leq m\right\}$ is open and dense in $A_{1}$. This implies that $f_{a_{2}}$ is a coneneighborhood isometry (similar to (3.9)). Then the claim follows from Lemma 3.8 (note that $\Sigma_{a_{2}} A_{2} \subseteq\left(f_{a_{2}}\left(A_{1}\right)\right)^{\geq \frac{\pi}{2}}$ in $\Sigma_{a_{2}} A$ by the first variation formula).

Proof of (6.4.2).

We first consider the case where $\operatorname{dim}\left(X_{2}\right)=0$, i.e., $X_{2}=\left\{p_{2}\right\}$ because $\partial X_{2} \neq \emptyset$. By (2.1.1), $m=1$ or 2. If $m=1$, then $X$ is isometric to a half suspension (see Corollary 6.3); if $m=2,\left(\Sigma_{p_{1}} X_{1}\right)^{\perp}$ consists of two points with distance $\pi$. In any case, we have that $\Sigma_{p_{1}} X=\left(\Sigma_{p_{1}} X_{1}\right) *\left(\Sigma_{p_{1}} X_{1}\right)^{\perp}$.

We now assume that $\operatorname{dim}\left(X_{2}\right)>0$, so $\operatorname{dim}\left(\left(\Sigma_{p_{1}} X_{1}\right)^{\perp}\right)>0$ by (6.4.1). Note that in $\Sigma_{p_{1}} X, \partial \Sigma_{p_{1}} X_{1}=\emptyset$ and $\lambda_{\zeta \xi}=1$ for any $\zeta \in\left(\left(\Sigma_{p_{1}} X_{1}\right)^{\perp}\right)^{\circ}$ and $\xi \in \Sigma_{p_{1}} X_{1}$ (see (2.11)). In view of Lemma 6.6 with (6.4.1) and Key Lemma 1.7, it suffices to show $\lambda_{\uparrow_{p_{1}}^{p_{2}} \xi} \leq m$ for any $\left[p_{1} p_{2}\right]$ with $p_{2} \in \partial X_{2}$ and $\xi \in \Sigma_{p_{1}} X_{1}$; and for any $\eta \in \Sigma_{p_{1}} X$, there is $[\zeta \xi]$ with $\zeta \in\left(\Sigma_{p_{1}} X_{1}\right)^{\perp}$ and $\xi \in \Sigma_{p_{1}} X_{1}$ such that $\eta \in[\zeta \xi]$.

Let $p_{2} \in \partial X_{2}$. By Lemma 6.5, 6.1 and 1.4, we can conclude that for any $\left[p_{1} p_{1}^{\prime}\right] \subset X_{1}$, there are at most $m$ minimal geodesics between $\uparrow_{p_{1}}^{p_{2}}$ and $\uparrow_{p_{1}}^{p_{1}^{\prime}}$. I.e., for any $\zeta \in\left(\Sigma_{p_{1}} X_{1}\right)^{\perp}$ (see (6.4.1)), $\lambda_{\zeta \xi} \leq m$ for any $\xi \in\left(\Sigma_{p_{1}} X_{1}\right)^{\prime}$, so for any $\xi \in \Sigma_{p_{1}} X_{1}$ by Lemma 2.2 .

Next, by Corollary 6.3 , any $x \in X$ belongs to some $\left[p_{1}^{\prime \prime} p_{2}^{\prime \prime}\right]$ with $p_{i}^{\prime \prime} \in X_{i}$. Following the argument in the proof of (6.4.1), we can conclude that $\uparrow_{p_{1}}^{x}$ belongs to some $\left[\uparrow_{p_{1}}^{p_{1}^{\prime \prime}} \uparrow_{p_{1}}^{p_{2}^{\prime \prime}}\right]$. This implies that any $\eta \in \Sigma_{p_{1}} X$ belongs to some $[\zeta \xi]$ with $\zeta \in\left(\Sigma_{p_{1}} X_{1}\right)^{\perp}$ and $\xi \in \Sigma_{p_{1}} X_{1}$.

Remark 6.7. For $p_{2} \in \partial X_{2}$, a priori it is not clear that $X_{1}^{m} \subseteq\left\{p_{1} \in X_{1} \mid \lambda_{p_{1} p_{2}}=\right.$ $m_{p_{2}}$ \} (see the end of Lemma 6.1). By Lemma 1.4 and (6.4.2), it is not hard to see that this is true.

We are now ready to conclude the proof of Theorem B.

\section{Proof of Theorem $B$.}

In our case where $\partial X_{2} \neq \emptyset$, the properties from Lemma 6.1 to Remark 6.7 guarantee that the arguments in the proofs of Lemma 4.2-4.6 will go through with minor modifications. And the proof of (A2) also works through here when $\operatorname{dim}\left(X_{2}\right)>0$. So it remains to consider the case where $\operatorname{dim}\left(X_{2}\right)=0$, i.e., $X_{2}=\left\{p_{2}\right\}$.

By (2.1.1), we have that $\lambda_{p_{1} p_{2}} \leq m$ for all $p_{1} \in X_{1}$ with $m=1$ or 2 . If $m=1$, then $X$ is a half suspension of $X_{1}$ (see Corollary 6.3). If $m=2$, then there is an obvious involution on $\Sigma_{p_{2}} X$, which is an isometry (similar to $\hat{\gamma}$ in (4.8)), and on $\left(\Sigma_{p_{1}} X_{1}\right)^{\perp}=\left\{\left(\uparrow_{p_{1}}^{p_{2}}\right)_{1},\left(\uparrow_{p_{1}}^{p_{2}}\right)_{2}\right\}$ for any $p_{1} \in X_{1}^{m}$. Then it is not hard to see that $X=\left(\Sigma_{p_{2}} X *\left(\Sigma_{p_{1}} X_{1}\right)^{\perp}\right) / \mathbb{Z}_{2}=S\left(\Sigma_{p_{2}} X\right) / \mathbb{Z}_{2}\left(\right.$ and $\left.X_{1}=\left(\Sigma_{p_{2}} X\right) / \mathbb{Z}_{2}\right)$.

\section{REFERENCES}

[AKP] S. Alexander, V. Kapovitach, A. Petrunin, Alexandrov geometry, In preparation.

[BGS] W. Ballmann, M. Gromov; V. Schroeder, Manifolds of nonpositive sectional curvature, Birkhäuser Boston Inc., Boston, Mass., (1985).

[BGP] Yu. Burago, M. Gromov, and G. Perel'man, A.D. Alexandrov spaces with curvature bounded below, Uspeckhi Mat. Nank 47(2) (1992), 3-51. 
[Ch] S. Y. Cheng, Eigenvalue comparison theorems and its geometric applications, Math. Z 143 (1975), 289-297.

[Fr] T. Frankel, Manifolds of positive curvature, Pacific J. Math. 11 (1961), 165-174.

[GG] D. Gromoll, K. Grove, A generalization of Berger's rigidity theorem for positively curved manifolds, Ann. Scient. Ec. Norm. Sup. 20(4) (1987), 227-239.

[GM] K. Grove, S. Markvorsen, New Extremal Problems for the Riemannian Recognition Program via Alexandrov Geometry, J. of AMS 8(1) (1995), 1-28.

[GS] K. Grove, K. Shiohama, A generalized sphere theorem, Ann. of Math. 106 (1977), 201211.

[Li] Xueping Li, Nonnegatively curved Alexandrov spaces with souls of codimension two, Trans. Amer. Math. Soc. 367 (2015), 3901-3928.

[Per] G. Perelman, Proof of the soul conjecture of Cheeger and Gromoll, J. Diff. Geom. 40(1) (1994), 209-212.

[Ro] X. Rong, Positively curved manifolds with abelian symmetry, Proc. of ICCM 2007, Higher Education Press and International Press, Beijing-Boston IV (2007), 195-229.

[RW] X. Rong; Y. Wang, Perel'man's Soul conjecture in Alexandrov geometry with souls of codimension 2 (In preparation).

[SSW] X. Su, H. Sun, Y. Wang, An Isometrical $\mathbb{C P}^{n}$-Theorem, Preprimt.

[Wi] B. Wilking, Index parity of closed geodesics and rigidity of Hopf fibrations, Invent. Math. 144 (2001), 281-295.

[Ya] T. Yamaguchi, Collapsing 4-manifolds under a lower curvature bound (2002).

Mathematics Department, Capital normal University, Beijing, P.R.C. Mathematics Department, Rutgers University, New Brunswick, NJ 08903, U.S.A

E-mail address: rong@math.rutgers.edu

School of Mathematical Sciences (and Lab. math. Com. Sys.), Beijing Normal University, Beijing, 100875 P.R.C.

E-mail address: wyusheng@bnu.edu.cn 